\title{
An Assessment of the Utility of a Non-Metric Digital Camera for Measuring Standing Trees
}

\author{
by \\ Neil A. Clark \\ Thesis submitted to the Faculty of the \\ Virginia Polytechnic Institute and State University \\ in partial fulfillment of the requirements for the degree of \\ MASTERS OF SCIENCE
}

IN

FORESTRY

APPROVED:

Randolph H. Wynne

Daniel L. Schmoldt

Richard G. Oderwald

September 1998

Blacksburg, Virginia 


\title{
An Assessment of the Utility of a Non-Metric Digital Camera for Measuring Standing Trees
}

\author{
by \\ Neil A. Clark \\ Randolph H. Wynne, Chair \\ Forestry
}

\begin{abstract}
(ABSTRACT)
A method is set forth which makes use of a commercially available, non-metric, solid-state matrix camera to capture spatial and spectral data from an individual tree bole that can be used to measure characteristics about the stem. In this study diameters and heights were measured and used to estimate the volume of 20 red oak (Quercus spp.) stems ranging in diameter at breast height from 16 to over $60 \mathrm{~cm}$ and height from 12 to 20 meters. Images were taken from four orthogonal directions around the each stem. Diameter estimates from matching camera to stem distances ( 3 to 15 meters) of opposite sides were arithmetically averaged. Two arithmetic averages from perpendicular directions were then geometrically averaged. It was found that locating the camera farther from the tree led to more consistent results over the entire stem while locating it closer to the tree provided the most precise estimates provided the inclination angle did not exceed 45 degrees.

This method resulted in geometric mean diameter estimates within $\pm 4 \mathrm{~cm}$ for all heights combined when obtained at a distance of $12 \mathrm{~m}$ or greater using a $95 \%$ chi-square maximum anticipated error statistic. Error increases with increased stem height from $\pm 3 \mathrm{~cm}$ to $\pm 7 \mathrm{~cm}$ for heights from 1 to 20 meters. In general, the error is equivalent to 3 times the instrument precision, which varies with distance. Two-thirds of the time volume estimates were within 8 percent, which is quite an improvement over the 30 percent interval afforded by an appropriate volume equation.
\end{abstract}




\section{ACKNOWLEDGMENTS}

I would like to thank all of the organizations and individuals who played a part in this research and my education. I would like to thank the USDA Forest Service Southern Research Station and the Department of Forestry at Virginia Tech in general for their financial and material support. I am thankful for Dan Schmoldt and Phil Araman who proposed the initial concepts of this project and oversaw much of the administration as well as provided guidance and technical expertise along the way. I thank my committee members: Dan Schmoldt, Randy Wynne, and Rich Oderwald for investing their time in revising all of my drafts and papers along the way and for sharing wisdom gained from their years of research with me. I wish to thank Connie Noonkester for taking care of a large amount of administrative issues and for going above and beyond the call of duty in dealing with "pesky" vendors. And I thank Dave Loftis and the crew at Bent Creek in Asheville and Matt Winn for field data assistance that would have otherwise been impossible.

My debt to my parents, Wade and Donna Clark and Janice and H. R. Hildenbrand, is inexpressable. I thank them all for their support, encouragement, and love throughout my life. I am also very greatful for the rest of my family, brothers and sisters, grandparents, aunts and uncles who have in many ways all played a part in bringing all of this to fruition. My wife, Laura, deserves an extra portion of thankfulness being as she had to take the brunt of being the wife of a graduate student (though it was her idea).

Lastly, and most importantly, thanks to Jesus Christ in whom we live, and move, and have our being (ACT 17:28), and who holds all things together (COL 1:17). Without Him all else is meaningless. I pray that this work and my life would be glorifying to Him. 


\section{TABLE OF CONTENTS}

Table of Figures...................................................................................................vii

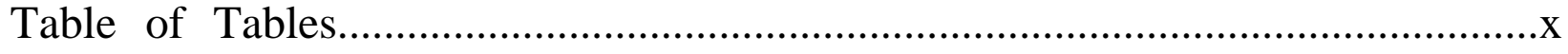

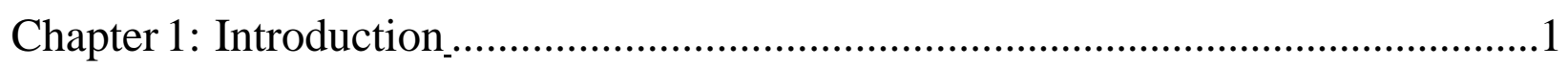

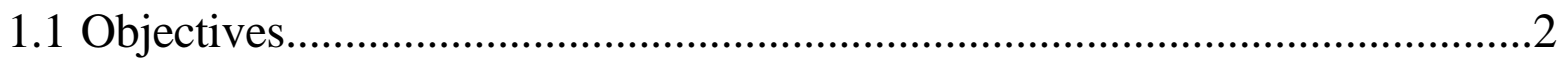

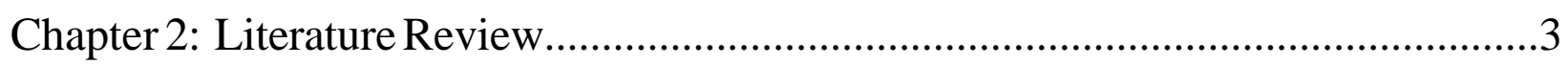

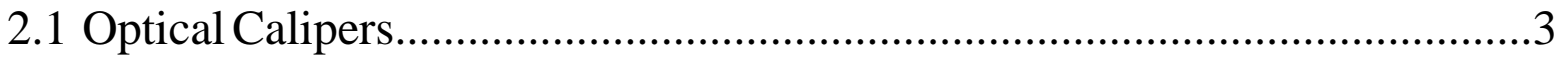

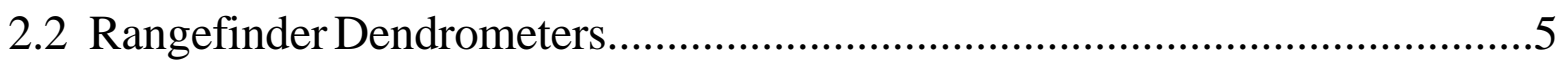

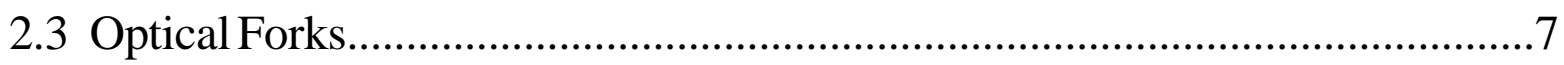

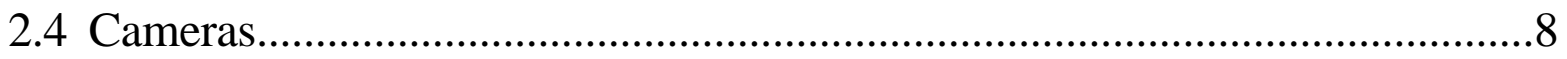

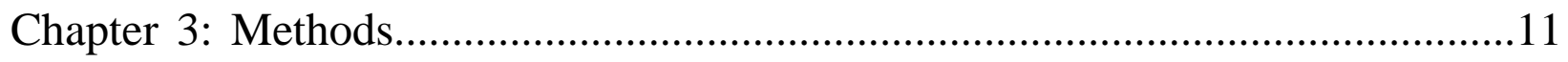

3.1 Determining Image Pixel Size .............................................................. 11

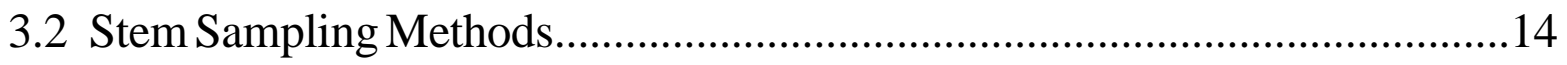

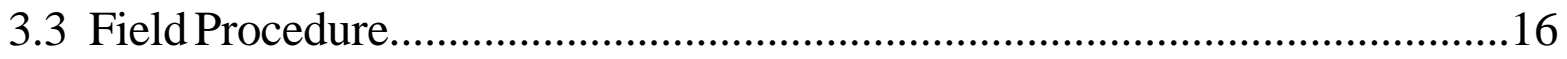

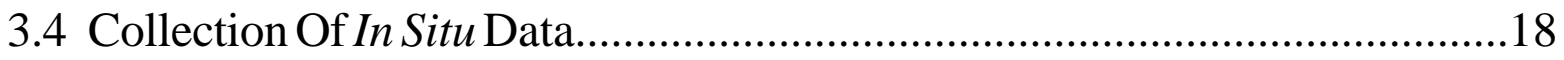

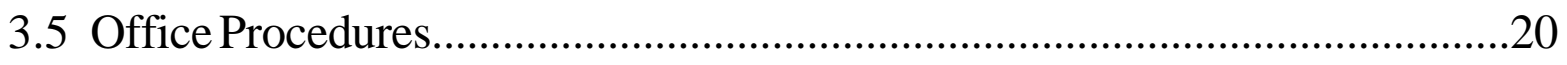

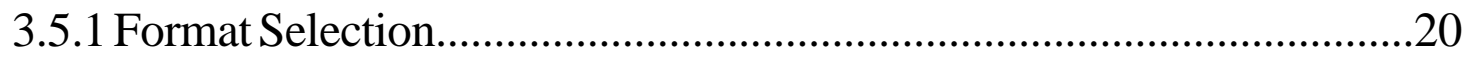

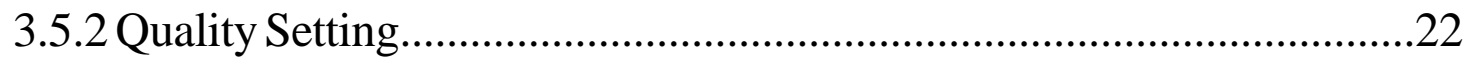




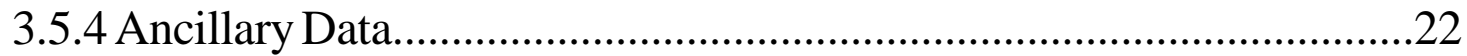

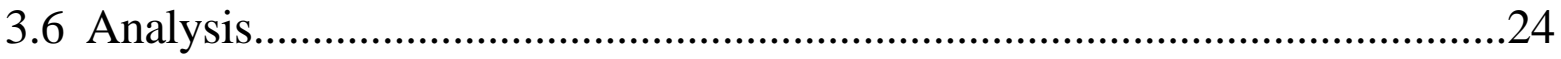

Chapter 4: Results and Discussion.............................................................26

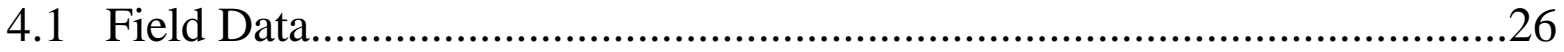

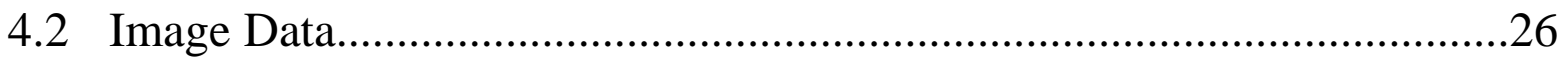

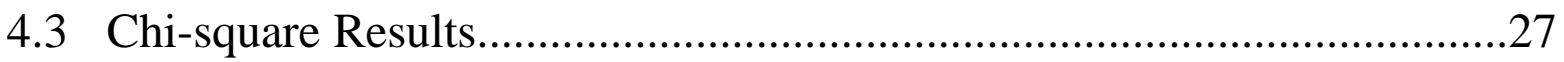

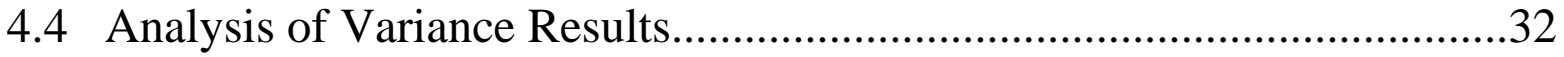

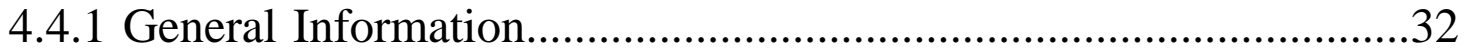

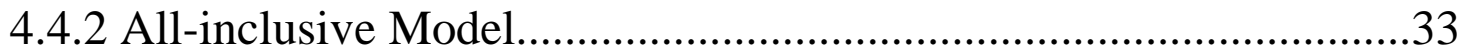

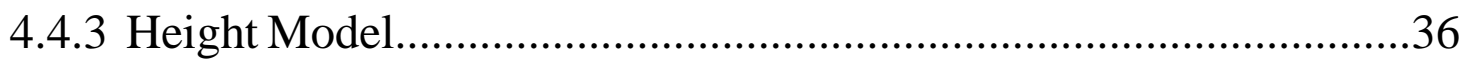

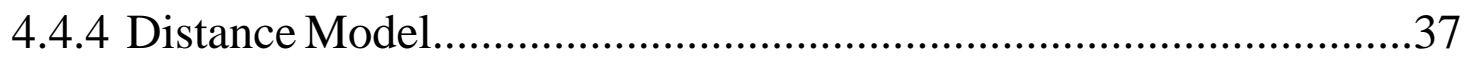

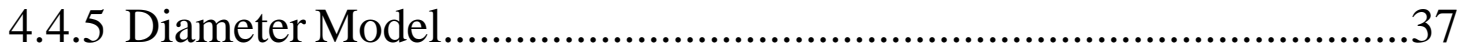

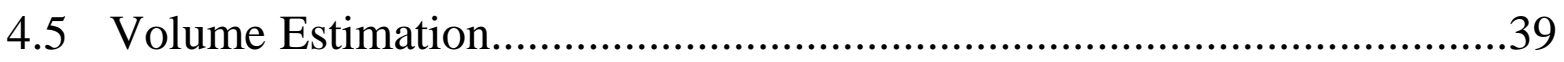

4.5.1 Volume Estimation Comparison.........................................................40

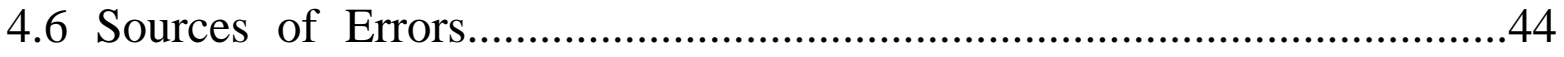

4.6.1 Mislocation Error...........................................................................44

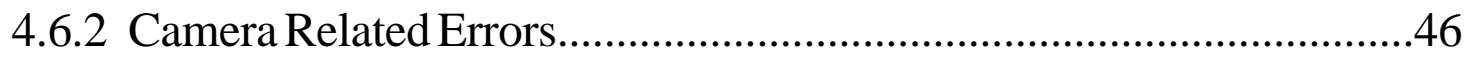

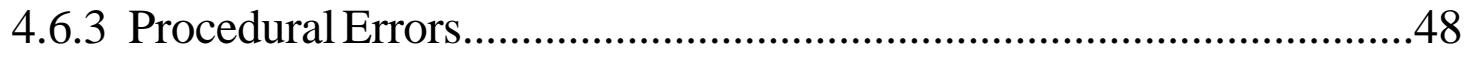


Chapter 5: Conclusion...............................................................................50

5.1 Collection Protocol.........................................................................................50

5.1.1 Image Scale.....................................................................................

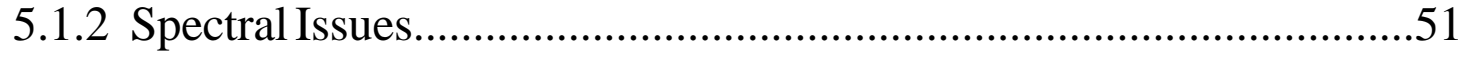

5.1.3 Seasonal Consideration.......................................................................52

5.2 Comparison to Results of Other Studies..........................................................52

5.3 Areas For Further Development...................................................................5

5.4 Summary and Conclusion.......................................................................58

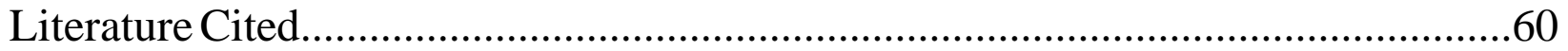

Appendix A - Measurement Derivation..........................................................................63

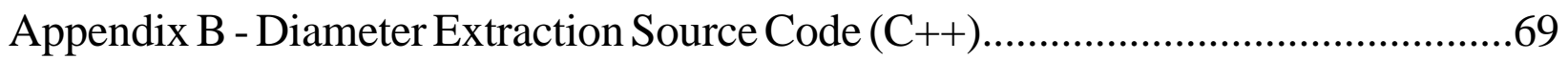

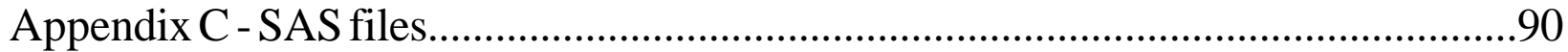




\section{TABLE OF FIGURES}

Figure 1. Means of measurements of various optical dendrometer types. The baseline distance

(b) is adjusted and substituted as a direct measurement of diameter for the optical calipers (distance is not necessary). Two distances $(d 1 \& d 2)$ and a baseline distance $(b)$ measurement are required for measurement using optical forks. Baseline distance $(b)$ is set with the rangefinder dendrometers. True and false convergence angles are used to calculate distance and ultimately, diameter......................................................6

Figure 2. False diameter error and oblique line of sight error recognized and corrected for by

Crosby et al. (1983)......................................................................................10

Figure 3. Illustration showing the components and operation of a CCD array. The silicone dioxide insulator provide the vertical confinement of the incident energy. The doped polysilicon electrodes provide the horizontal confinement and release electrons into the collection region. The electron bundles corresponding to each pixel are transferred to a processor which measures the incident energy and assigns it to a spatial location. Coupled with information about the filter array, further processing can be done to

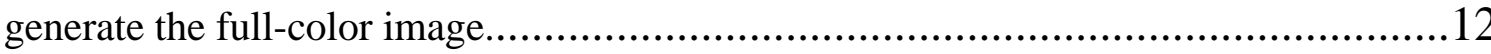

Figure 4. Measurement precision based on pixel size for the Kodak DC-120 digital camera.

Figure 5. Orientation of caliper measurements and camera stations for procedure used in this study......

Figure 6. Flowchart of field data capture procedure....................................................19

Figure 7. Office procedure used to extract information from the images............................21

Figure 8. Upper and lower images at single camera station showing location and image row value of $B_{o}$ and identifiable point $a$ and row values for each of the images.............23

Figure 9a-b. Normal probability plots and frequency histograms of camera minus caliper geometric mean diameter estimates. Probability plots also report Kolmogorov-Smirnov

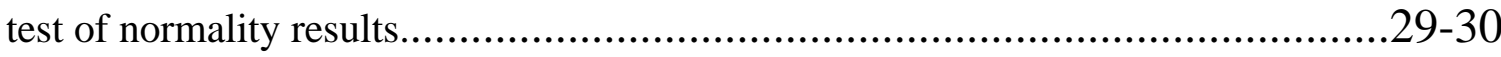

Figure 10. Normal probability plots and frequency histograms of camera minus caliper geometric mean diameter estimates grouped by stem height. Probability plots also report 
Figure 11. Example of butt swell on a $60 \mathrm{~cm} \mathrm{DBH}$ red oak. Bottom left is cross section at 0.3 $\mathrm{m}$ above ground, top left is cross section at $1.5 \mathrm{~m}$ above ground diagramed to indicate locations of points used by calipers and differing camera distances (exaggerated for explanatory purposes). This extreme case shows the potential change in size and form of a stem in the first 2 meters.

Figure 12. Lower section of stem at 3 and 15 meter camera stations (left to right). Pixel resolution at the edge of the stem at the flagging is 2.2 and 11 millimeter for the 3 and 15

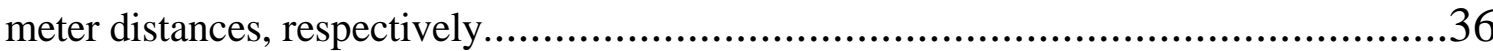

Figure 13. Scatterplots of geometric mean diameter error for 3, 6, 9, 12, and 15 meter distances and all distances combined.

Figure 14. Location of points for a given diameter at various distances from two diametrical directions. The locations are consistent within each direction but not between directions.

Figure 15. Perpendicular view of same stem shown in Figure 14 depicting the problems inherent in determining edge points on a forked stem

Figure 16. Diagram showing error increase due to loss of precision.

Figure 17. Magnified portion of image showing the edge of a stem shifted by differing background spectral properties. The outlined region represents the indiscrete region containing the edge of the stem

Figure 18. Two examples of a backlit condition. The left image demonstrates the technique of blocking the direct radiation and using an appropriate shutter speed to capture reflectance from the stem. This image can later be enhanced to more clearly distinguish the captured reflectance values. The right image is exposed to direct radiation which inundates the reflective radiation disallowing any useful spectral data collection.

Figure 19. Tree lean error using the averaging method complicated by deliquescent branching. The lines represent the locations of the camera estimates used to estimate diameters that were actually located within the polygon regions......................................54

Figure 20. The camera captures data that can potentially be used to model stem defects........57 
Figure A-1. Diagram of object space showing measured or calculated angles, distances and

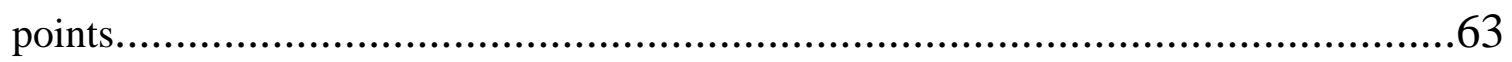

Figure A-2. Image space showing relationship of angle $\phi$ and image points $B_{\text {ileft }}$ and $B_{\text {iright }} \ldots .65$ 


\section{TABLE OF TABLES}

Table 1. Summary table of empirical studies of various optical dendrometers.......................4

Table 2. Kodak DC120 quality setting options and corresponding file sizes and disk

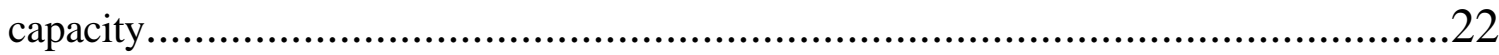

Table 3. Number of field and image collected diameter measurements obtained from 20 red oak stems ( 8 in Radford, Virginia / 12 in Asheville, North Carolina) in March/April 1998.

Table 4. Summary statistics for 241 camera minus caliper geometric mean diameter estimates in centimeters by distance and diameter for 20 red oak stems. Reported are the arithmetic means and standard deviations, median, mode, paired $t$-value test statistics, the maximum anticipated error (E) using the chi-square test for accuracy, and the actual measurement range of the nearest $95 \%$ of the observations from the median observation..................32

Table 5. Summary statistics for volume estimates by distance and diameter for 20 red oak stems. Reported are the arithmetic means, standard deviations, and the maximum anticipated error (E) using the chi-square test for accuracy

Table 6. Volume estimates for 20 red oak stems comparing camera estimates from the 12 meter distance, the average of all camera estimates, and the equation estimates to the "true" caliper estimates. The left four columns show the stem volumes in cubic meters, the next three columns from the left show the cubic meter differences, and the final three columns report the percent differences

Table 7. Site comparison of 12 meter camera distance, average of camera distances, and equation volume estimates for 12 red oak stems on North Carolina site versus 8 red oak stems on Virginia site 


\section{Chapter 1}

\section{INTRODUCTION}

Measurements of trees are taken for the determination of growth, volume/biomass estimation, health, value assessment, habitat evaluation, etc., all of which come into play in making resource management decisions. Height and diameter measurements usually form the foundation of most analyses involving the health, volume, growth, change, and potential of a tree or stand of trees. Dendrometers are instruments used to measure tree diameters. The most accurate and reliable dendrometers contact the stem at predetermined points of measurement. Formulation of volume equations, intensive inventories (Cost 1979), and some sampling strategies (Gregoire et al. 1987, Van Deusen and Lynch 1987) require measurement of diameters that are not easily accessible. Direct measurement of these points can be costly, both in time and money. And given that most work needs to be done in a given time frame, and within a given budget, it is very important to collect these data as accurately and efficiently as possible. Climbing the tree or felling the stem is labor intensive, dangerous, and sometimes incompatible with the desired objectives. For instance, cutting the tree would not allow a tree to be remeasured at a later date, and spikes that are often used for climbing will affect future stem growth, precluding unbiased repeated measurements. A dendrometer must be efficient and not disrupt a stem's normal growth in addition to being "simple to use, portable, relatively inexpensive, accurate at all tree heights, and operable independently of distance from point of measurement" (Avery and Burkhart 1983). Optical dendrometers, which allow measurements to be taken visually from a remote location, have been developed to fill this niche.

This study explores the feasibility of using a non-metric digital camera for tree mensuration. Conventional cameras have been used for this purpose in the past with mixed 
results (Marsh 1952, Ashley and Roger 1969, Bradshaw 1972, Crosby et al. 1983, and Takahashi 1997). Problems that have been mentioned include the following: the expense of film and development, the time delay due to development, complicated calculations, improper exposure, and problems of orientation. Changes in technology have brought about the re-emergence of the camera's usefulness for the purposes of mensuration.

The manufacturing and medical industries actively use digital imagery to rapidly and accurately measure products with great precision (Clarke 1995). The camera used in this study has a liquid crystal display (LCD) that allows the image to be visualized seconds after acquisition. If the exposure settings were not correct, the picture can be erased, exposure adjusted, and image reacquired. Within limits the image can be enhanced back in the office in order to detect more information. However, in severe cases of under- or over-exposure, enhancement does not help. Digital images can be manipulated and measured faster, easier, and with less expensive equipment than conventional photographs. The problem of the complicated calculations can be mitigated using algorithms imbedded in image processing software. Camera orientation can be determined by means of digital clinometers and rangefinders. There is a one time expense for the purchase of the camera and storage disks, but disks can be reused, eliminating the continual cost of film and printing supplies.

\subsection{Objectives}

This study assessed the utility of non-metric digital cameras for tree mensuration. The specific objectives were as follows:

1. Develop and test the methods required for obtaining accurate tree stem measurements 
2. Set forth protocols for data collection

3. Field test the unit under "realistic" conditions to verify the utility of protocols and processing methods

4. Compare the results of this system to other optical dendrometers commonly used

5. Compare volumes determined by the method set forth in this paper using the camera to those using other volume equations

6. Identify areas for further development

Chapter 2

\section{LITERATURE REVIEW}

Time savings and practicality have been the engines driving the development of optical dendrometers. Many styles of optical dendrometers have been designed based on a number of different principles. Three of these principles are forks, calipers, and rangefinders (Grosenbaugh 1963). In order to measure a diameter optically two lines of sight must exist between the observer and two points on the stem lying in the plane representing the desired diameter. The following sections discuss each type in further detail and Table 1 shows summary information for some empirical studies using these optical dendrometers.

\subsection{Optical Calipers}

Optical calipers use two parallel lines of sight to view points on a stem that represent the diameter. The McClure mirror caliper (McClure 1969) and the Wheeler (1962) pentaprism are two examples of optical calipers. These instruments provide direct readings for the same points that a mechanical caliper would contact. This characteristic eliminates location error (which occurs using the other types of optical dendrometers) caused by diameter calculation using observation points that are not the same as the mechanical caliper contact points. The other 


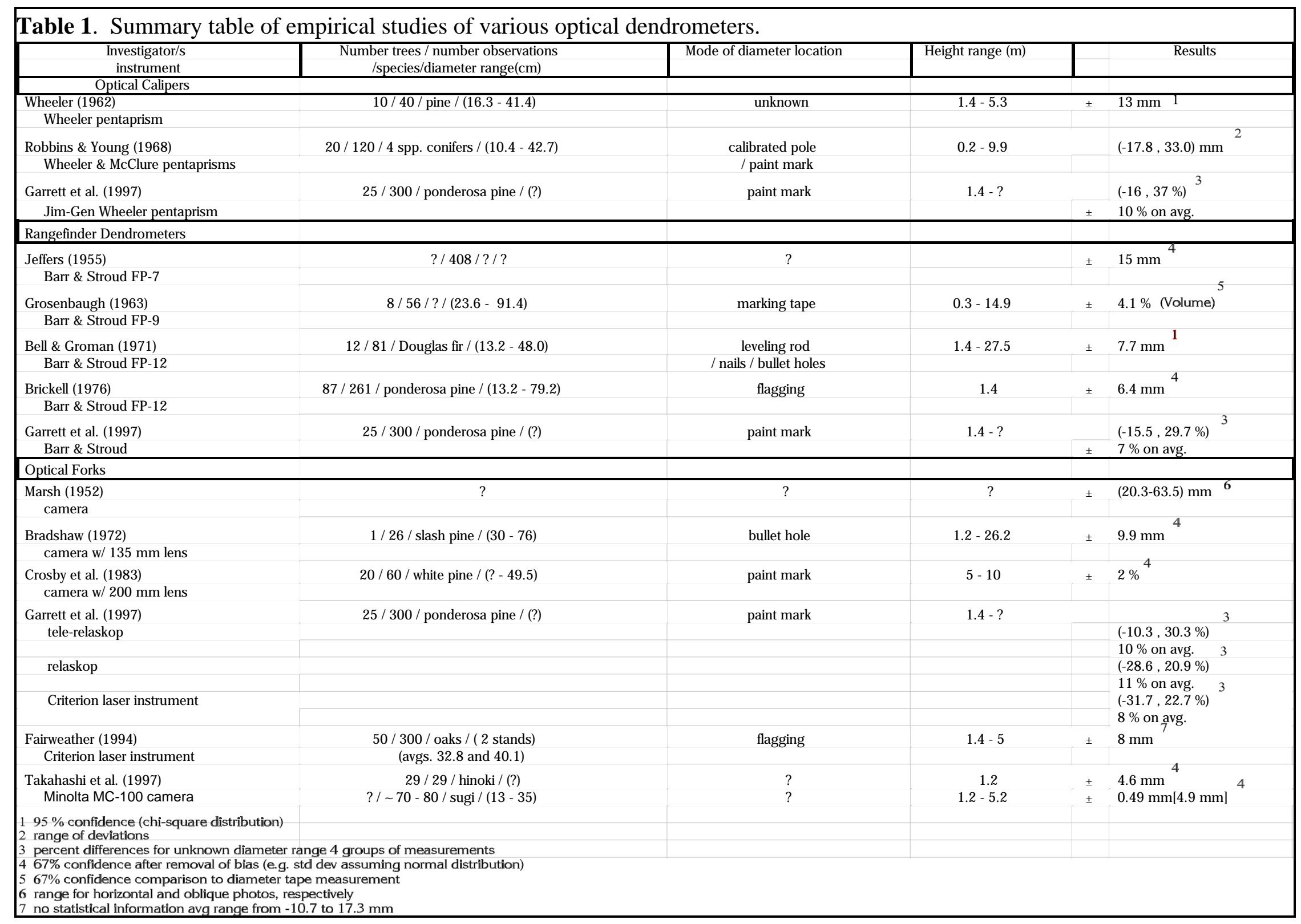


advantage to this direct reading is that it does not require knowledge of line of sight distance. The disadvantage is that upper diameters are usually referenced by a height measurement which requires the addition of a hypsometer, an instrument for measuring tree heights. Since this angular measurement is not intrinsic to the caliper procedure additional time is required to take the measurement, and depending on the hypsometer used, requires consideration of tree lean, distance determination, etc. Diameters are limited to the instrument's length (usually $91 \mathrm{~cm}$ ), though longer less portable versions can easily be constructed (Grosenbaugh 1963).

In a pilot test of ten trees at two heights (1.4 and $5.3 \mathrm{~m}$ ), Wheeler's pentaprism caliper measurements were within $\pm 13 \mathrm{~mm}$ of wooden caliper measurements using a 95 percent chisquare test (Wheeler 1962). Robbins and Young (1968) compared the Wheeler pentaprism, an early version of the McClure pentaprism (that only permitted direct readings to the nearest 13 $\mathrm{mm}$ ), and a diameter tape to conventional calipers. Twenty stems were used, containing marked diameters at 6 heights (from 1.5 to 10 meters). Ranges of differences from conventional calipers were $(-18,33),(-18,20)$, and $(-20,25) \mathrm{mm}$ for diameter tape, McClure and Wheeler pentaprisms, respectively. All instruments studied had a slight positive bias between 3 and $5 \mathrm{~mm}$.

\subsection{Rangefinder Dendrometers}

The remaining two types of optical dendrometers have lines of sight that intersect, so diameters are not direct measurements, but rather a calculation of an average radius which is then doubled to estimate diameter. The rangefinder defines a baseline distance that separates two lines of sight. One line is set tangent to one side of the stem and the other angle of the line of sight is adjusted until the points coincide. A true coincidence and false coincidence (Figure 1) are obtained in order to calculate a radius that represents the stem's diameter when doubled.

Excellent histories of the development of the Barr and Stroud dendrometer can be found 
in Grosenbaugh (1963) and Brickell (1976). Brickell (1976) reports that in "field conditions" (level terrain with little underbrush and excellent light conditions), two-thirds of the dendrometer measurements were within $0.64 \mathrm{~cm}$ of the caliper measurements for diameters between 12.7 and $76.2 \mathrm{~cm}$. Bell and Groman (1971) report a $\pm 6.9 \mathrm{~mm}$ result for a 95 percent chi-square test for observations at seven heights on twelve Douglas fir (Pseudotsuga menziesii) stems ranging in DBH (diameter at breast height) from 25.4 to $48 \mathrm{~cm}$. Jeffers (1955) obtained results of \pm 15.2 $\mathrm{mm}$ for diameter at a 95 percent confidence level in his field test with an added caveat that standard deviations three times larger may be common where visibility is obscured.

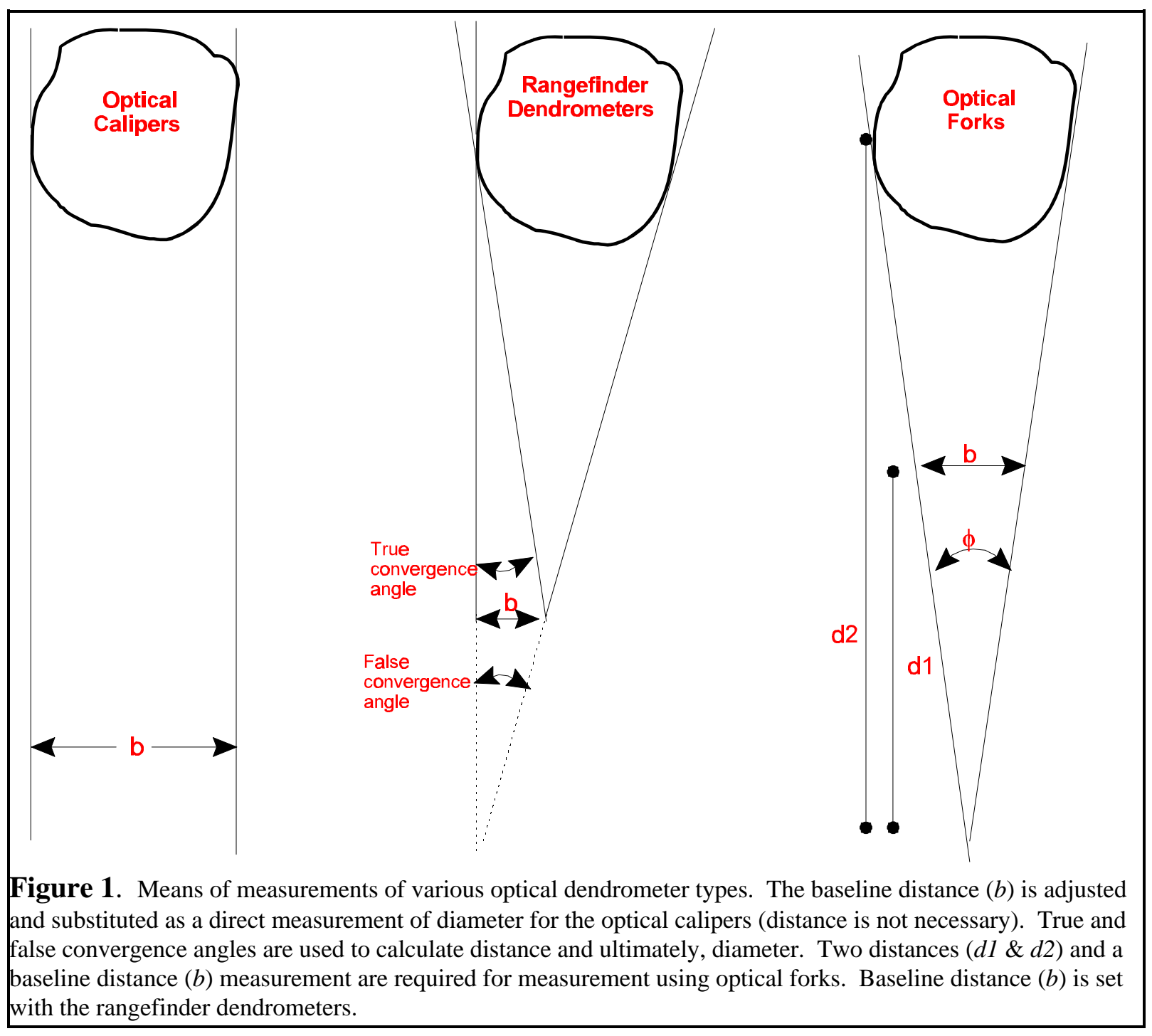




\subsection{Optical Forks}

Optical forks are instruments where the two lines of sight intersect at a point in front of the observer. The angle of the two lines of sight tangent to the stem is measured using a scale at known distance from the point where the lines of sight intersect. Since these forks work off of the principle of similar triangles, distance to the point of measurement (range) needs to be obtained. Often, an angular measurement is used in conjunction with a distance measurement to another point in order to calculate the range.

Some of these dendrometers work with a fixed fork angle, such as a wedge prism mounted to one lens of an ordinary pair of binoculars (Bitterlich 1984). A wedge prism used with an angle gauge has also produced satisfactory results (Ashley and Roger 1969, Rennie and Leake 1997). The observer must adjust the sighting distance from the stem until the limiting distance of the wedge is approached. Distance and angles must then be measured in order to calculate a correct diameter. Although the components of this "instrument" are inexpensive, implementation is challenging.

Other optical forks vary or measure the line of sight angle. Military binoculars having a mil-scale (Forbes 1955) have been used to accomplish this purpose. The Liljenström, Zeiss, and the Mesavage teletops are other examples of instruments that work on this principle. More information on these instruments can be found in Brickell (1976) and Grosenbaugh (1963). The Spiegel Relascope (Rennie and Leake 1997) and the Tele-relaskop (Parker 1997) are two commercially available dendrometers based on Bitterlich's angle count concept. The multipurpose relascope has not faired well when used to measure upper stem diameters (Garrett et al. 1997, Ashley and Roger 1969, Rennie and Leake 1997). Relascope errors are credited to the lack of magnification, which is where the Tele-relaskop with its 8x magnification picks up. 
The latter instrument has been shown to be capable for diameters (Garrett et al. 1997, Parker 1997), but not very useful for height determination (Williams et al. 1994).

\subsection{Cameras}

Cameras fit into the optical fork category. Rather than measuring the angle at a point between the intersection of the lines of sight and the diameter being measured, a measurement is taken from an image. The image is formed from light rays that expose some medium at the same angle as they entered the lens. Marsh (1952) provided some of the first results of using terrestrial photogrammetry to measure tree diameters. His reported results were not very good, $\pm 63.5 \mathrm{~mm}$ for oblique photos and $\pm 20.3 \mathrm{~mm}$ for horizontal photos. Lack of visual clarity, computational complexity, and cost in dollars and in time were reasons further development of the device was suspended at that time (Grosenbaugh 1963).

Ashley and Roger picked up this idea in 1969 and designed a device and procedure that placed the camera in a set orientation to the stem ensuring that the stem axis was in the principal plane of the photo and that the depression angle (the stem axis is defined as the horizon) was parallel to the longitudinal axis of the stem (Ashley and Roger 1969). The device was equipped with four moveable "points" that could be adjusted to represent this longitudinal axis. They presented both the mathematical and graphical techniques for calculating diameters using the perspective grid theory for high oblique photography. They mention that tree bend does not have a great effect so long as the plane is in one direction and that the camera is placed on the stem perpendicular to that bend. They did not present any field test results of this device, however, and reported laboratory measurements of placed targets (every 5 feet up to 100 feet on a flat surface) to be $\pm 7.6 \mathrm{~mm}$.

A simpler method using a camera with a $135 \mathrm{~mm}$ lens in conjunction with a clinometer 
and tape for calculation of slope distance was presented by Bradshaw (1972). Diameter

estimates were calculated using equation 1.

$$
D=k d S
$$

where $D=$ estimated tree diameter

$k=$ constant for lens and measuring system

$d=$ photo measured diameter

$S=$ slope distance from camera to point of measurement

From this equation there does not appear to be any correction for the interior orientation of the image plane. Twenty-six diameters ranging from $30-76 \mathrm{~cm}$ at points from 1.2 to 26 meters in height were measured with an accuracy of $\pm 9.9 \mathrm{~mm}$. Tree lean again became somewhat of an issue due to the method of slope distance calculation.

Another study using a $200 \mathrm{~mm}$ lens and a scale of known length was done by Crosby et al. (1983). The scale was raised with a telescoping rod to the heights of 5, 7, and 10 meters. Horizontal versus vertical scale orientation, as well as placement of the scale in front versus beside the stem was studied. The best results were achieved with the horizontal orientation placed in front of the stem. This method eliminated the necessity of knowing the slope distance, thus eliminating the effect of tree lean. A correction factor was introduced in order to offset biases created by (1) false diameter observation due to non-coincidence between lines of sight and the actual diameter and (2) the oblique line of sight that results in a photo-measured diameter that is actually higher on the stem than the desired point of measurement (Figure 2). This correction factor requires a paced horizontal distance from the camera to the stem, thus eliminating some of the advantages of this technique. Average errors reported by this study were 0.063 percent and 0.089 percent for black and white photos and slides, respectively, with standard deviations of 1.91 and 2.40 percent on diameters less than $50 \mathrm{~cm}$. Some of the 


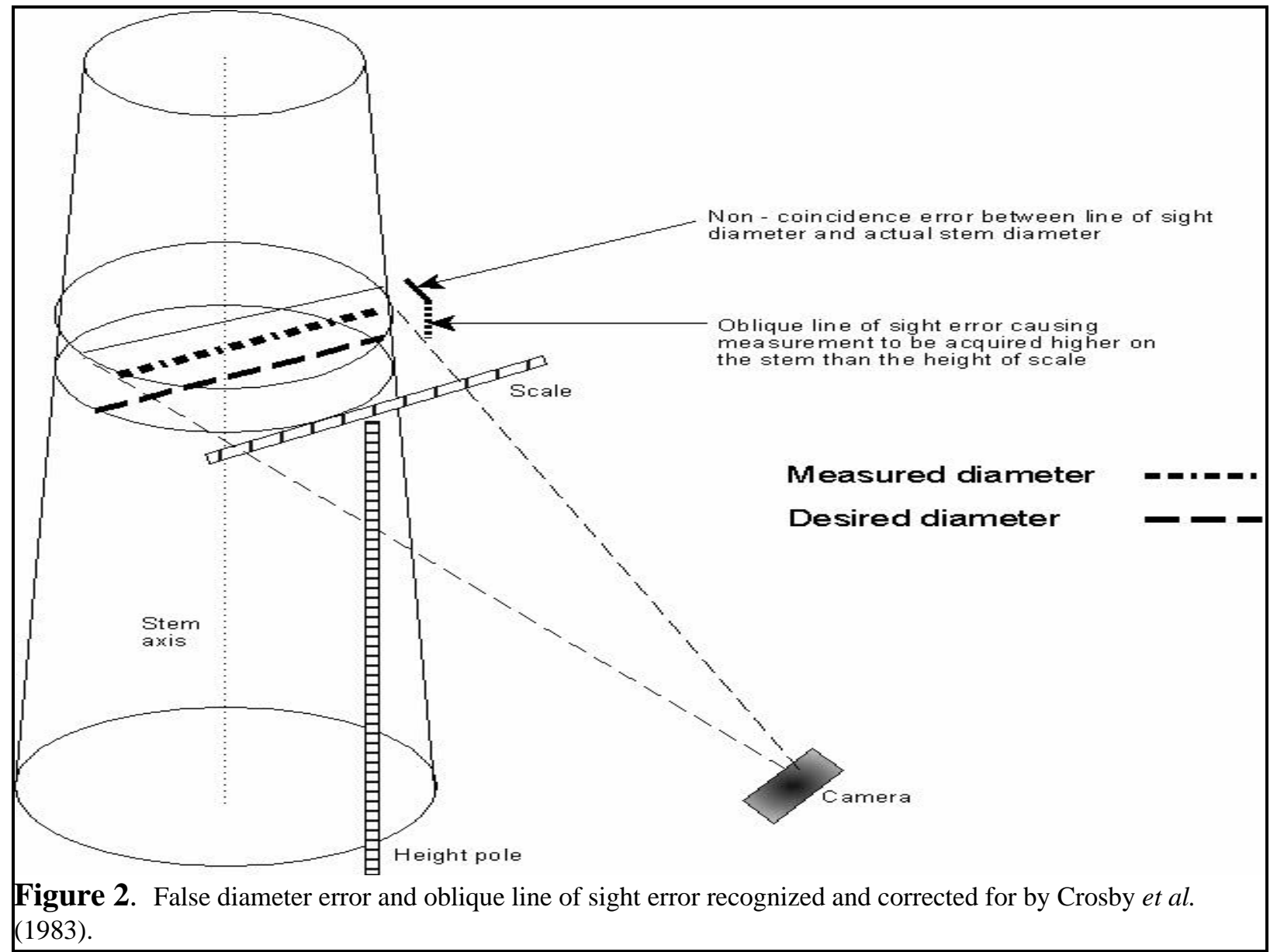

drawbacks for this system are the need for two persons during data collection and the height restriction of the telescoping rod, which was $10 \mathrm{~m}$ in this case.

Minolta finally broke some ground with their development of a prototype camera that uses its focus capability as a rangefinder (Takahashi et al. 1997). In the study by Takahashi et. al (1997), this prototype produced results with mean error of $+0.15 \mathrm{~mm}$ with a standard deviation of $0.49 \mathrm{~mm}$, after corrections for false diameter and distance adjustment, on sugi (Cryptomeria japonica) with diameters between 13 and 35 centimeters and heights of 1.2, 3.2, and 5.2 meters. In another experiment, 29 diameter measurements of hinoki (Chamaecyparis obtusa) stems were obtained with a mean error of $+1.6 \mathrm{~mm}$ and standard deviation of $4.6 \mathrm{~mm}$ after corrections for bark and systematic errors. This study presents the difficulty of obtaining measurements in dark 
conditions.

From the literature so far, it appears that $\pm 5 \mathrm{~mm}$ is about the best level of accuracy that can be obtained with optical dendrometers. The average level obtained is twice that amount. Though many of these instruments have been tested separately under differing conditions and using different forms of analysis, there have been few published direct comparison tests. One recent publication (Garrett et al. 1997) compares the Barr and Stroud dendrometer, the Telerelaskop, Jim-Gen Wheeler pentaprism, relaskop, and the Criterion 400 laser instrument under summer and winter conditions. A description and contemporary pricing information is presented for each instrument. DBH and upper stem diameters of unreported heights were measured with calipers on twenty-five ponderosa pine (Pinus ponderosa) trees with calipers and with each of the dendrometers by three observers. There was between a 5 and 12 percent difference $(\alpha=0.05)$ between each of the dendrometers and the caliper measurements. This percent difference was calculated using the sum of squares of percent differences in order to prevent cancellation of the positive and negative deviations. The Barr and Stroud dendrometer seemed to perform the best with other instruments being about equal.

\section{Chapter 3}

\section{METHODS}

\subsection{Determining Image Pixel Size}

The resolution of a conventional photograph is determined by the "grain" of the film, more specifically the reactant chemicals in the film emulsion. The Kodak DC-120 digital camera uses an $850 \times 984$ element CCD (charge-coupled device) array to produce a resultant image that is $960 \times 1280$ image pixels. CCDs are silicon devices comprised of an array of potential wells created through a series of column implants (vertical confinement) and electrodes (horizontal 


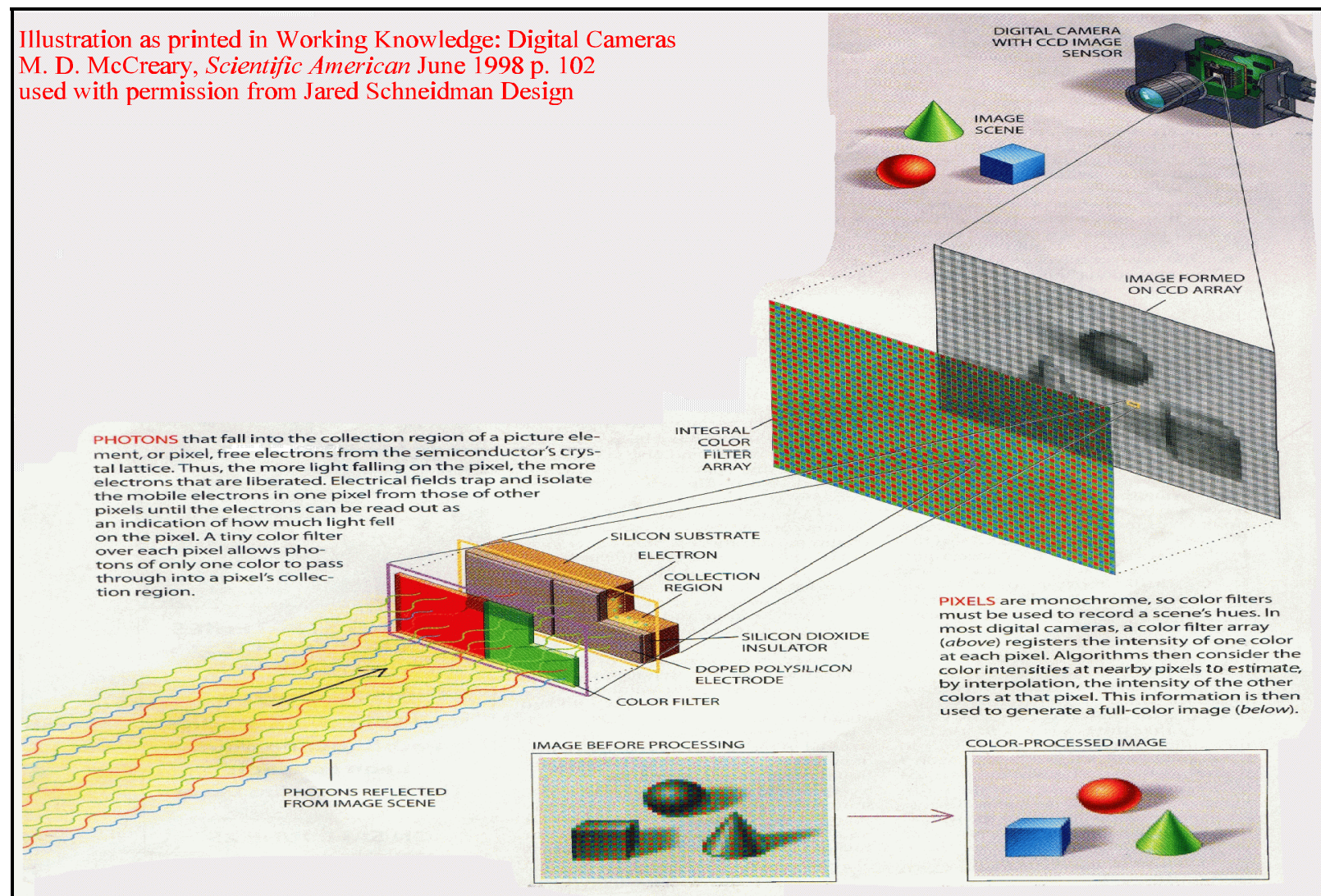

Figure 3. Illustration showing the components and operation of a CCD array. The silicone dioxide insulator provide the vertical confinement of the incident energy. The doped polysilicon electrodes provide the horizontal confinement and release electrons into the collection region. The electron bundles corresponding to each pixel are transferred to a processor which measures the incident energy and assigns it to a spatial location. Coupled with information about the filter array, further processing can be done to generate the full-color image.

confinement)(Holland 1997)(Figure 3). In other words, photons enter the system for a

predetermined length of time and strike a color filter array which is used to limit the bandwidth

of the energy incident upon each sensing element (Evans and Hardwick 1997). Electrons are

shaken loose from the electrodes in each of the sensing elements and are collected into "packets"

in the potential wells. Each element in the DC120 CCD array is 7.8 x 5.0 microns in dimension

(Kodak 1997). These 836,400 packets are then transformed to produce the final 3,686,400

digital output values $(960 \times 1280$ (pixels) $\times 3$ (colors)). The details of this transformation are

proprietary, precluding a priori knowledge of image pixel size. However, image pixel size had

to be determined in order to obtain accurate measurements. 
To determine the size of the image pixels, a rectangular light post (assumed to be plumb) was imaged at nine distances ranging from $0.3-21.6 \mathrm{~m}$, keeping the camera axis horizontal.

$$
\begin{aligned}
& s=\frac{d}{D} \\
& s=\frac{f}{L_{o}}
\end{aligned}
$$

Equations 2 and 3 show two equations that were used for scale determination, where $s$ represents scale, $d$ is the image measurement representing the real world length $D, L_{o}$ is the horizontal distance between the lens and the object and $f$ is the focal length of the camera. $D$ was measured using a steel tape to the precision of $\pm 1.6 \mathrm{~mm}, L_{o}$ was measured with a nylon tape $\pm 12.7 \mathrm{~mm}$, and $f$ as reported by the camera manufacturer is $7 \mathrm{~mm}$ (at maximum wide angle). $d$ was solved for and divided by the average number of pixels representing each measurement. These estimates of the pixel widths from the nine distances were again averaged and this average was rounded to the nearest micron. The result is 5 microns, which was used as the image pixel dimension in all subsequent calculations. This calculation was made only along the column direction, and an assumption is made that the image pixels are square. Because much of the underlying processes are proprietary, the camera's actual precision is unknown and only the precision of the calculated image pixel can be determined.

Because the image consists of discrete pixels, image measurements can only be made to a precision of \pm 1 pixel without performing some interpolative techniques. Assuming all of the image pixel dimensions were equal, the precision would then be represented in object space as a linear function of the distance from the imaged object. Equations 2 and 3 are used in this case 


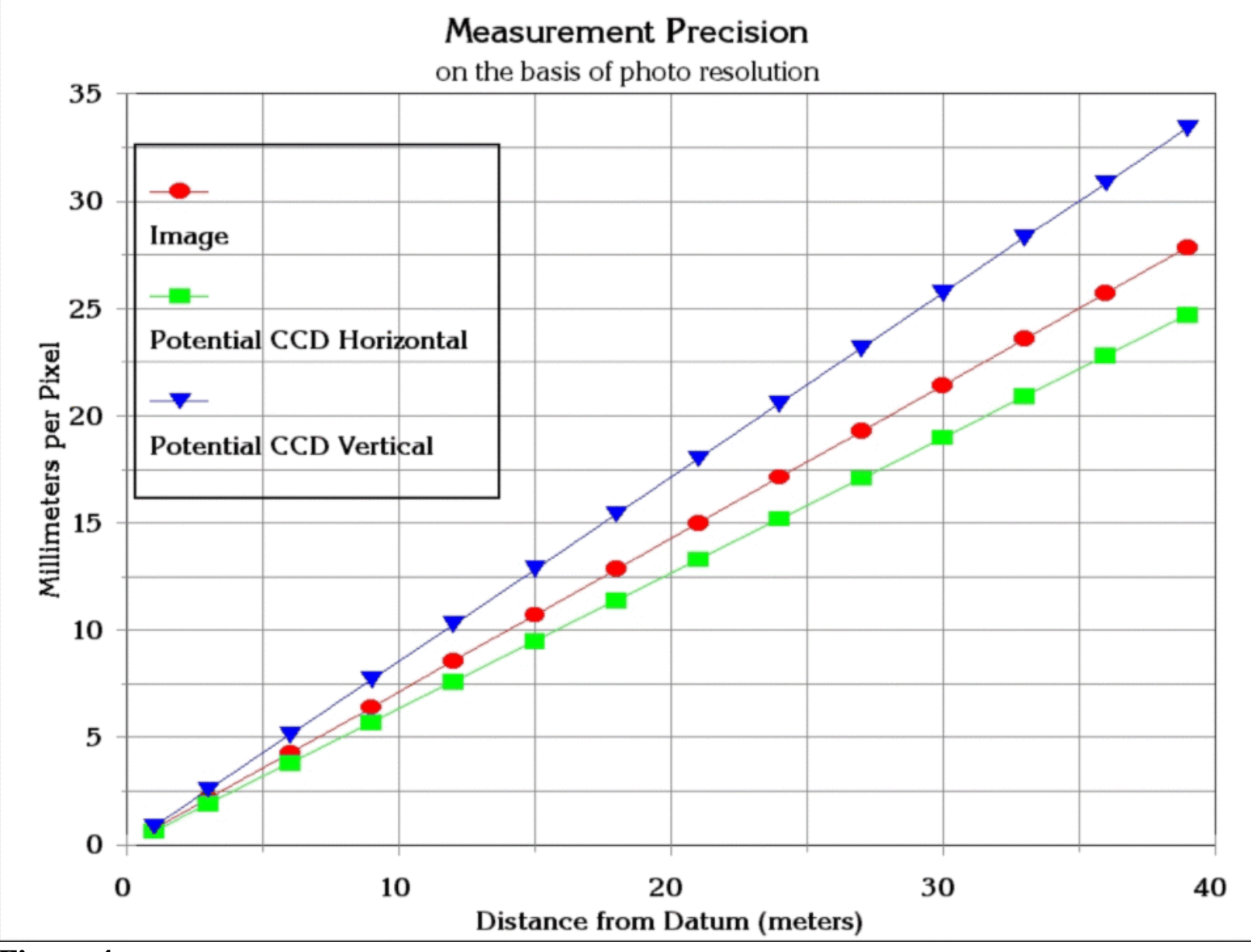

Figure 4. Measurement precision based on pixel size for the Kodak DC-120 digital camera.

with $d$ being equal to 5 microns, $L_{o}$ set at 1 meter, $f$ equal to $7 \mathrm{~mm}$, and solving for $D$ which equals $\pm 0.7 \mathrm{~mm}$ per meter from the object (Figure 4 ).

\subsection{Stem Sampling Methods}

To carry out the objectives of this study a sample design was selected to enable statistical comparisons to be made among different procedures and with the results of previous studies. Many studies were vague about sampling procedures and sample design. Given that many different tools and techniques were used in different locations, duplication is difficult. For example, many studies did not indicate what species were sampled and under what conditions. In some cases, species was a consideration. In the study by Binot et al. (1995), species 
type was considered due to the differing effects of bark roughness on the ultrasonic waves that were recorded by the electronic tree measuring fork. Takahashi et al. (1997) correct for bark roughness before reporting their results.

Another consideration associated with species is the cross sectional deviation from a circle. Some species such as yellow poplar (Liriodendron tulipifera) tend to deviate less from a circular cross section than others, e.g., chestnut oak (Quercus montana). Some species also tend to vary more in cross sectional shape than others. Obviously there are other phenotypical interactions such as aspect, successional state, physical damage, etc. that have their effects as well. These were not included in our experimental controls.

Hence, red oak (Quercus spp.) was selected due to its moderate variations in bark and form distinctions. Northern red oak (Quercus rubra), black oak (Quercus velutina), and scarlet oak (Quercus coccinea) were the species sampled in this study. This species criterion was imposed to reduce variation due to morphological dissimilarity among species.

One of the goals of this study was to set forth a data collection protocol. This would depend predominantly on the required data. If only a single diameter measurement is needed, a single image using maximum zoom is appropriate, along with accounting for tree lean. In order to collect data for an entire stem much of the procedure has already been developed (Clark et al. 1998).

A strength of the camera is the amount of data that can be collected per unit time. For this reason the shortest focal length was used to capture the maximum length of stem per image. The distance between the stem and the camera station was the main component that was to be investigated. Inversely related to this is the angle of inclination of the camera. To study the possible effects of this angle/distance relationship, data were captured at approximate distances 
of 3, 6.1, 9.1, 12.2, 15.2 meters. These distances correspond to 10, 20, 30, 40, 50 feet because the nylon tape used to measure distance was in English units.

At each stem a quick visual survey was administered to select four orthogonal directions with the least amount of obstruction from understory vegetation or parts of adjacent stems. A mark using red spray paint was made on one face of the stem corresponding with the direction determined with the visual survey. This mark was used as an indicator of the direction of the first camera station and aided in orientation of the caliper measurements after the stem was felled. The second camera station was directly opposite the mark, and the third and fourth camera stations were perpendicular to the mark (Figure 5).

\subsection{Field Procedure}

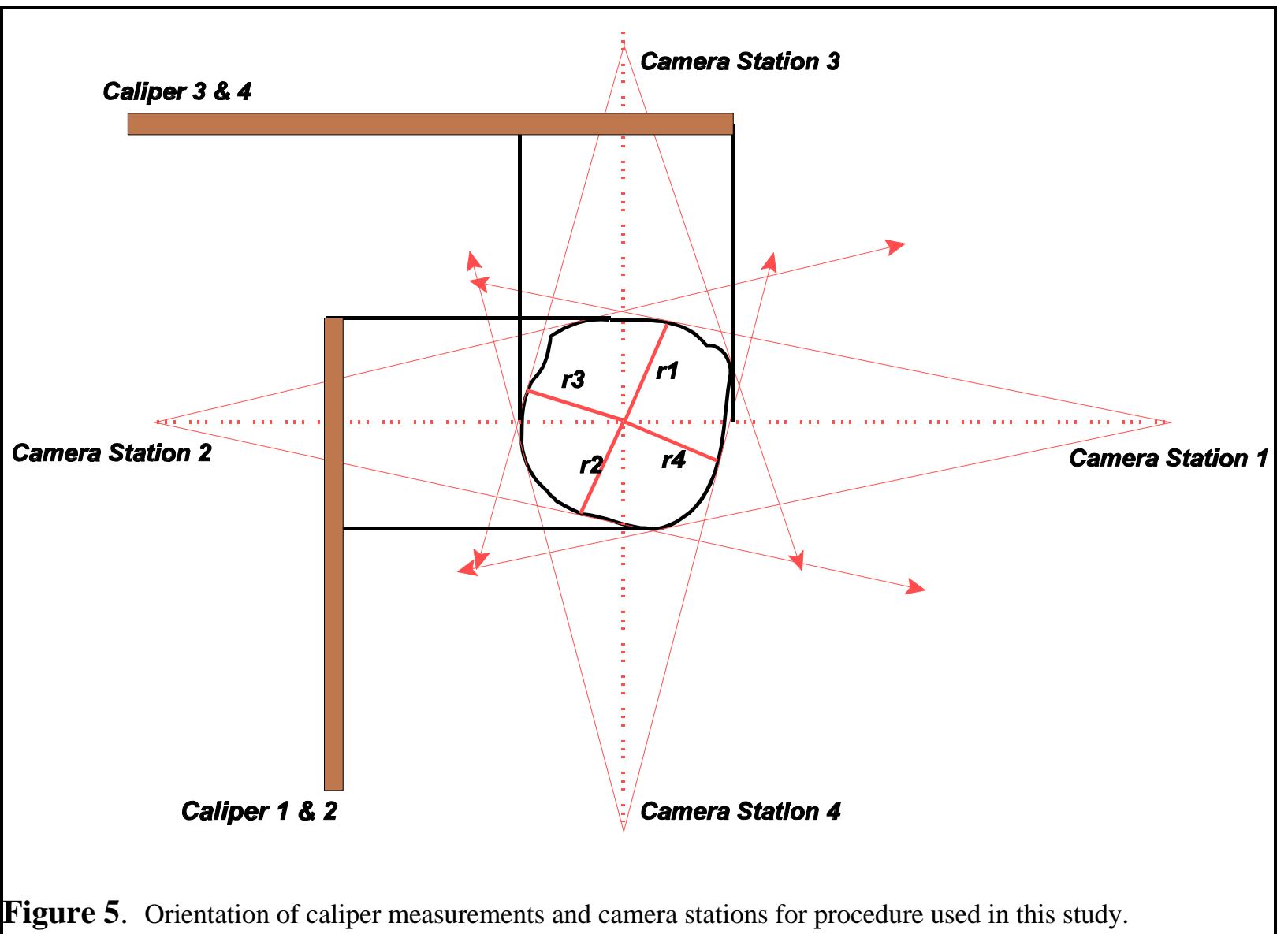


For each of the 20 camera stations (5 distances, 4 directions) at each stem, data were collected using the following procedure (Figure 6).

1. A steel tape was secured to the ground at the approximate midpoint of the tree stem in the plane formed by the camera station and the tangent of the stem parallel to the stem axis/camera station plane $\left(B_{o}\right)$ (Figure A-1). This same point was used for both diametrical camera stations. If the stem was on a slope, the uphill side was selected.

2. The DC120 was positioned on the tripod so that the widest dimension was vertical (e.g., with the button on the side) at a good vantage point for the portion of the stem that was to be imaged. The camera was then located at the appropriate distance from the stem along the predetermined line of sight.

3. For antipodal camera stations, the bearing of the opposing camera stations was determined by sighting the primary station and the stem.

4. Camera parameters were set such that:

- The focal length was set at maximum wide angle by pushing the $\mathbf{W}$ button until motor stopped.

- The focus was set to one point.

- The flash was deactivated.

- The shutter speed was set to auto-exposure +1.5 stops for most images. In backlit conditions the shutter speed was reduced and in lower light conditions the exposure time was increased.

- Image quality setting 3 was selected.

5. The tripod was set up as plumb as possible, but was not measured.

6. The stem was observed through the camera viewer and the camera oriented such that the base of the stem could be seen at the bottom of the viewer.

7. The inclination angle $(\theta)$ was recorded (Figure A-1).

8. The distance between the front center of the camera lens and the spot where the tape was secured at the base of the tree was measured to the nearest 0.5 inch and this distance $\left(L_{o}\right)$ recorded (Figure A-1). 
9. When the base image was captured successfully the LCD monitor was checked to see if the image was properly exposed. If not, adjustments were made and the image was recaptured.

10. An identifiable point on the stem or something in the background that is at the top of the viewer window was noted and used to regulate the position of the next image. The vertical clamp on the tripod was loosened and the camera inclined until the identifiable point was at the bottom of the window. The vertical clamp was secured and the next image captured.

11. This process was continued until the entire stem face was imaged. Then, after the opposing site was lined up and a marker placed, the camera station was moved and the process repeated.

This procedure was used in order to capture as much data as possible from one visit.

Obviously there is a cost in collecting data for the whole stem. Grosenbaugh (1963) sets forth magnification as a much needed factor for improved results. The Kodak DC120 has a 3x zoom capability allowing a $35 \mathrm{~mm}$ format equivalent focal length of $114 \mathrm{~mm}$. The actual focal length is just $21 \mathrm{~mm}$ owing to the small size of the CCD array. This does not approach the 500 mm lens used in the study by Takahashi et al. (1997), but is closer to the 135 and $200 \mathrm{~mm}$ lenses used by Bradshaw (1972) and Crosby et al. (1983), respectively. Images of selected single diameters were captured at this longer focal length, but the data were not analyzed in this study.

\subsection{Collection Of In Situ Data}

In situ data were collected destructively as a means of comparing the accuracy of image derived measurements. After all imagery was acquired, the stems were felled. Height measurements were made from the uphill contact point of the ground and stem. The downed stem was then measured with a nylon tape to determine the exact points at which to measure diameter. Steel calipers were used to measure diameters perpendicular to the camera station 


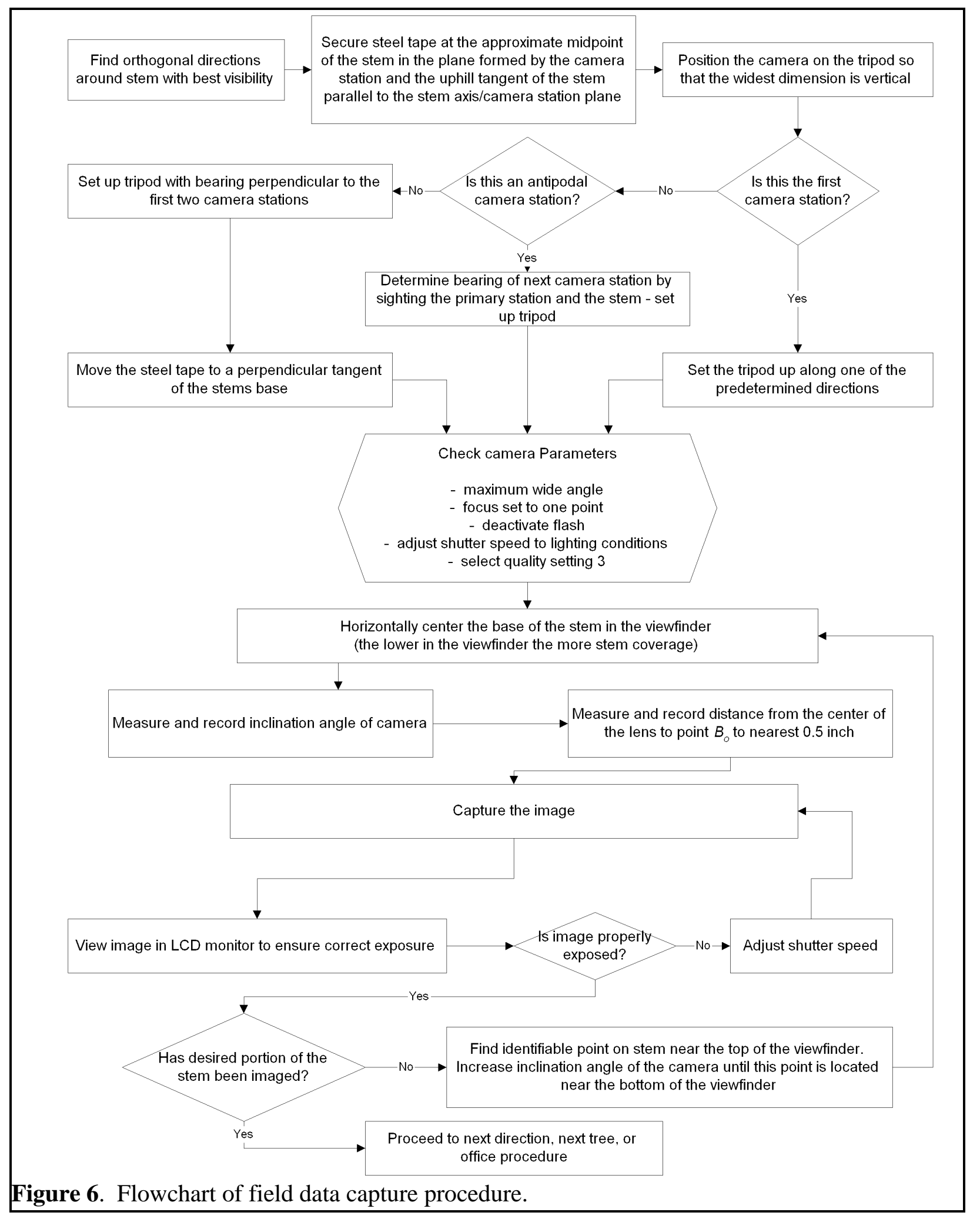


locations resulting in two perpendicular measurements at each height (Figure 5). This way one caliper measurement corresponded to two camera measurements for each directional diameter. Diameter measurements were taken at $1.4 \mathrm{~m}$ (diameter at breast height [DBH]) and every $1.2 \mathrm{~m}$ from the height of $2.4 \mathrm{~m}$ to a $10 \mathrm{~cm}$ top or to the end of the merchantable stem.

\subsection{Office Procedures}

Images were downloaded into a computer for processing (Figure 7). The Kodak Picture Transfer software that accompanied the camera was used to transfer the images from the camera to the hard disk on the computer.

\subsubsection{Format Selection}

The Kodak Picture Transfer software allows images to be transferred to the computer in different file formats. The $\mathrm{KDC}^{1}$ format, which is the format that is stored in the camera, was used to reduce the amount of storage space for archival purposes. This format also allows information such as the quality setting, exposure time, and date/time to be associated with each image. The stored images were then "acquired" using a TWAIN" module provided with the camera. The images were converted to tagged image file format $\left(\mathrm{TIFF}^{3}\right)$ as needed. TIFF was utilized as it is a standardized format that allows an image to be accepted by a variety of image processing packages. The images are 3,686 kilobytes when completely uncompressed to the

\footnotetext{
${ }^{1}$ Native Kodak Digital Camera proprietary format which allows excellent image compression without loss of data due to format translation.

${ }^{2}$ TWAIN (TWAIN 1998) software allows data captured from digital scanners, and in this case digital cameras, to be converted into data compatible for use with computer monitors and operating systems.

${ }^{3}$ Device independent, non-proprietary, standard file format created in 1986 by Aldus Corporation and scanner vendors. (Adobe 1998)
} 


\section{Office Procedures}

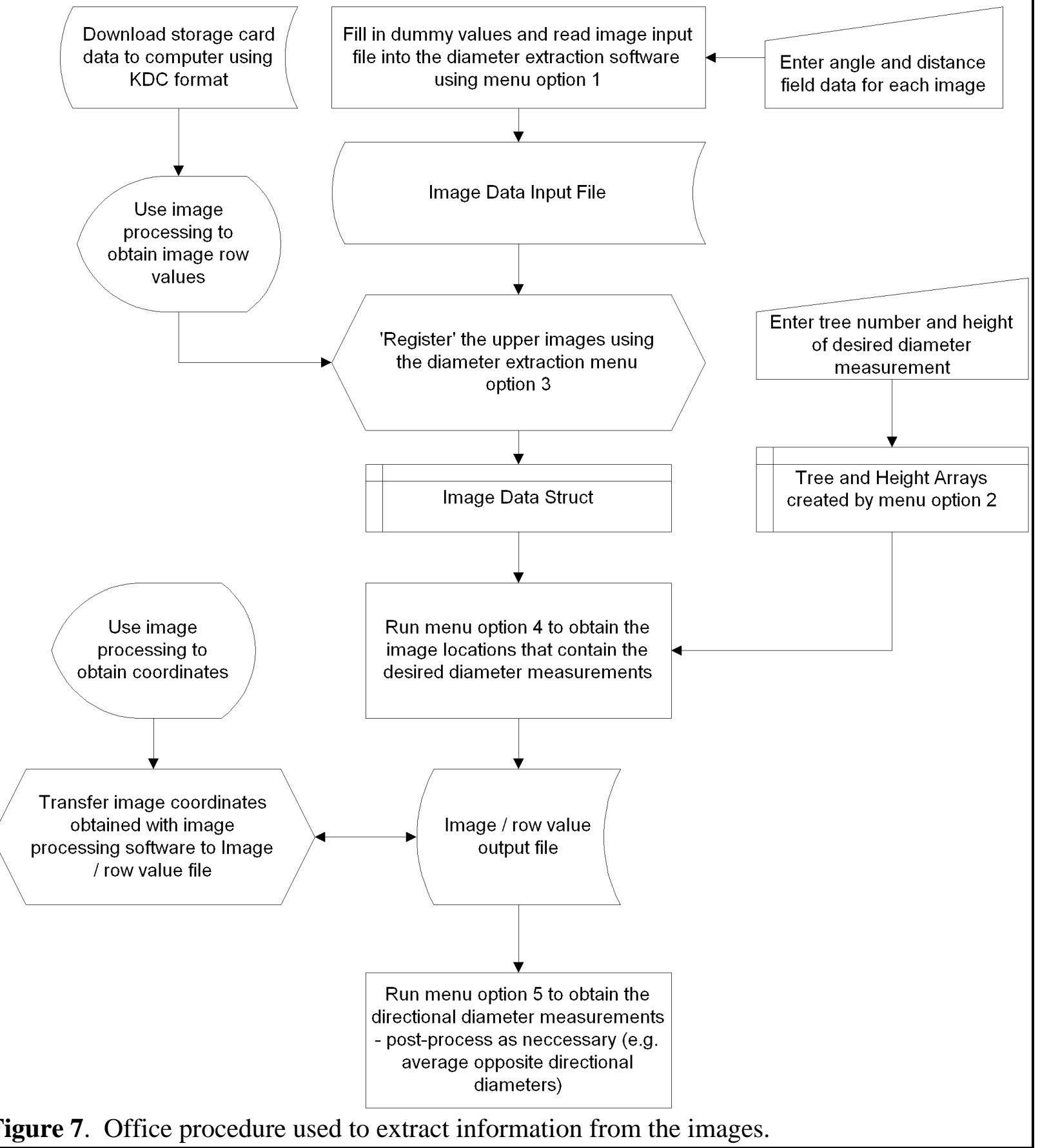


TIFF format.

\subsubsection{Quality Setting}

The DC120 allows the user to select from four image quality settings that spectrally compress the data to varying degrees while maintaining the same spatial resolution (Table 2). Quality setting number 3 was used in this study as it provided the best image "clarity" for a file size that was reasonable in both time for data collection and archival storage purposes.

\begin{tabular}{|ccc|}
\hline Table 2. Kodak DC120 quality setting options and corresponding file sizes and disk capacity \\
\hline Quality setting & \# per $10 \mathrm{mb}$ card & approximate file size \\
1 & 111 & $90 \mathrm{~kb}$ \\
2 & 71 & $140 \mathrm{~kb}$ \\
3 & 41 & $240 \mathrm{~kb}$ \\
4 & 12 & $830 \mathrm{~kb}$ \\
\hline
\end{tabular}

\subsubsection{Organizational Considerations}

The images were sequentially numbered by the camera in the order that they were captured. Great care was taken to ensure that the images remained in the same queue so that they could be matched up to the correct angle, distance, face, and stem field data. Image measurements in this study were obtained using ImageTool (University of Texas Health Science Center at San Antonio $1998^{4}$ ).

\subsubsection{Ancillary Data}

Ancillary data were entered into an ASCII file that was accessible to the diameter extraction software that was written for this project (Appendix B). For each image, tree number,

\footnotetext{
${ }^{4}$ Freeware available on the Internet by anonymous FTP from ftp://maxrad6.uthscsa.edu
} 
face number, image distance $\left(L_{o}\right)$, and the angle of inclination $(\theta)$ were transferred from field data sheets into this ASCII file. The image of the stem's base was viewed and the image row value representing $B_{o}$ was transmitted to the ASCII file and assigned a height value (Figure 8). Given that this was the base point, it was adjusted or estimated so that 0 was the highest point where the

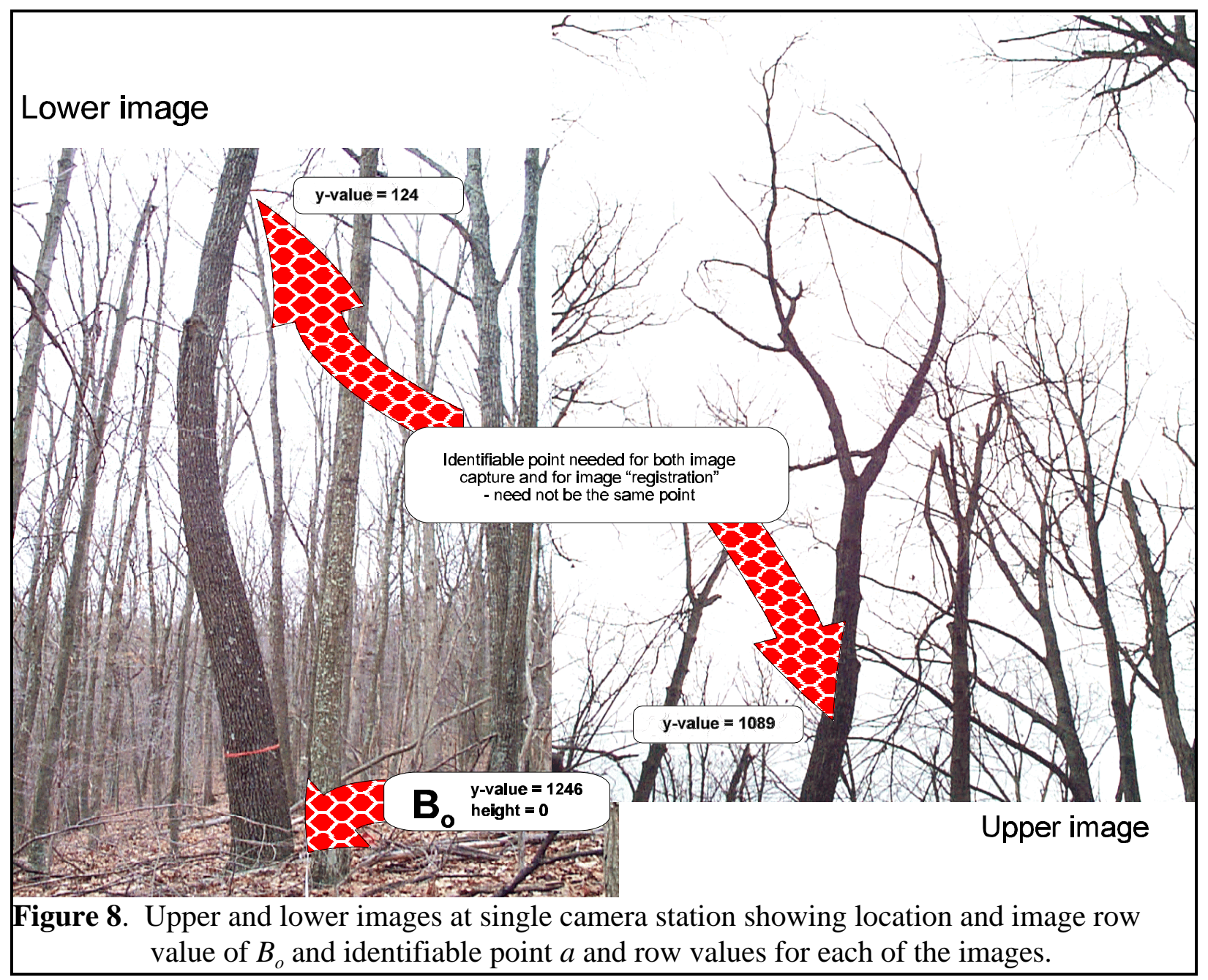

ground met the stem. On level ground it was possible that all four base images per stem could have been set to 0 , otherwise the uphill (the side where the tape was secured) side of the faces perpendicular to the slope was set equal to 0 . The base heights in the sloped directions were 
estimated by averaging the distance between the high and low sides of the images showing the slope and subtracting from 0.

For the upper-stem images, an identifiable point $(a)$ was chosen, and the height to this point was calculated (Appendix A) by a module in the diameter extraction software (Appendix B). This height was transferred to the ASCII file along with the corresponding row value from the upper stem image. $B_{o}$ for the upper image becomes the identifiable point $a$, with height calculated from the lower image. $L_{o}$ for the upper image becomes the distance from the focal point to $a$ and $\gamma$ (angle between line of sight to $B_{o}$ and a plumb line passing through the stem axis at the height of $B_{o}$ ) becomes $180^{\circ}$ minus the angle formed by the stem axis and the ray between the focal point and $a$ in the lower image.

Diameters were derived from the raw image data using the diameter extraction software written by the author (Appendix B). Tree numbers and stem heights of the desired diameters were also entered into an ASCII file. The program reads the ASCII file containing the field data and image information and the stem and height ASCII file and outputs the names of the images that contain the points that correspond to the desired diameters and their corresponding row values. The image coordinates representing the left and right edges of the stem at the respective row values were obtained with the ImageTool program and imported into an input file used by the diameter extraction software, which calculated the diameters using the methods described in Appendix A.

\subsection{Analysis}

There were two caliper measurements of diameter for each height, and four camera measurements of diameter for each height. The two diametrical camera measurements at 
matching distances were arithmetically averaged to produce a directional diameter. From this the geometric mean of the two perpendicular measurements for both the camera and the caliper were used to calculate a "pseudodiameter" of a circle with the same area as the ellipse with semimajor and semiminor axes $a$ and $b$ (Equation 4).

$$
G M D=\sqrt{a b}
$$

where $G M D=$ the geometric mean diameter

$a$ and $b=$ perpendicular directional diameters

Cubic foot volume (later converted to cubic meters) was then calculated using Smalian's formula (Equation 5) for each 1.2 meter (4 foot) bolt and summed to report the total merchantable volume for the stem. English units are used here for the convenience of comparison to existing volume tables.

$$
\text { Volume }=\frac{\pi}{4 \times 144} \frac{D_{l}^{2}+D_{s}^{2}}{2} l
$$

where $D_{l}=$ large end diameter (inches)

$D_{s}=$ small end diameter (inches)

$l=$ length of section (feet)

Though Smalian's formula has the tendency to overestimate volume, especially for the butt log, here it was used as a means of comparison rather than an accurate estimate of volume.

A chi-square test (Equation 6) was introduced by Freese (1960) to determine whether a technique meets a certain accuracy requirement. It was modified by Bell \& Groman (1971) to determine the maximum anticipated error. A chi-square test was used in this study to measure the anticipated accuracies at various distances and for different diameter classes. 


$$
E=\sqrt{\frac{\sum_{i=1}^{n}\left[\left(x_{i}-\mu_{i}\right)^{2}\right] \cdot r^{2}}{\chi_{n}^{2}}}
$$

where $x_{i}=$ camera estimate of the $i^{\text {th }}$ observational unit

$\mu^{i}=$ "true" estimate of the $i^{\text {th }}$ observational unit

$r^{2}=$ value of the standard normal deviate at a set $\alpha$ level

$\chi_{n}^{2}=$ chi-square value with $n$ degrees of freedom

Chapter 4

\section{RESULTS AND DISCUSSION}

\subsection{Field Data}

There were 241 paired perpendicular diameter measurements taken using conventional calipers in the field. Varying stem heights, damage incurred during felling, and human error accounted for unequal sample sizes within the various strata. Table 3 shows the number of field measurements taken by height category.

\subsection{Image Data}

Table 3 also shows the geometric mean diameter (GMD) estimates that were derived from the image measurements. Missing GMDs are the result of at least one of the four image measurements being absent. This usually occurred when the view of the stem was obstructed. This might be avoided in non-experimental situations where there are fewer restrictions on camera station location and tree height designation.

There were no great differences in measurement attainability from the 6 to the 15 meter distance class. The 3 meter distance numbers fall off sharply after about the 11 meter stem height mark, predominantly due to the sensitivity of the acute view angle to stem lean. 


\begin{tabular}{|c|c|c|c|c|c|c|}
\hline \multirow[b]{2}{*}{ Stem height $(\mathrm{m})$} & \multicolumn{5}{|c|}{ Nominal Distance of Camera Station (m) } & \multirow[b]{2}{*}{ field } \\
\hline & 3 & 6 & 9 & 12 & 15 & \\
\hline 1.4 & 18 & 18 & 18 & 18 & 18 & 18 \\
\hline 2.4 & 19 & 18 & 18 & 18 & 17 & 19 \\
\hline 3.7 & 19 & 19 & 19 & 19 & 19 & 19 \\
\hline 4.9 & 19 & 18 & 19 & 19 & 19 & 19 \\
\hline 6.1 & 17 & 18 & 18 & 18 & 18 & 19 \\
\hline 7.3 & 17 & 18 & 18 & 18 & 18 & 19 \\
\hline 8.5 & 17 & 18 & 18 & 18 & 18 & 19 \\
\hline 9.8 & 16 & 19 & 19 & 20 & 19 & 20 \\
\hline 11.0 & 12 & 18 & 20 & 20 & 19 & 20 \\
\hline 12.2 & 9 & 17 & 17 & 18 & 15 & 20 \\
\hline 13.4 & 4 & 11 & 14 & 14 & 10 & 15 \\
\hline 14.6 & 3 & 10 & 9 & 11 & 12 & 13 \\
\hline 15.8 & 2 & 6 & 7 & 8 & 9 & 10 \\
\hline 17.1 & 2 & 4 & 3 & 4 & 4 & 6 \\
\hline 18.3 & 0 & 2 & 1 & 0 & 1 & 3 \\
\hline \multirow[t]{2}{*}{19.5} & 0 & 0 & 1 & 0 & 1 & 2 \\
\hline & 174 & 214 & 219 & 223 & 217 & 241 \\
\hline
\end{tabular}

\subsection{Chi-Square Results}

When the inherent assumption of normality is not met using Freese's chi-square test of accuracy, Gregoire and Reynolds (1988) showed that considerable degradation of results can occur. For this reason the Kolmogorov-Smirnov test for normality was performed on the data groups prior to the application of the chi-square procedure. The results of the normality tests are shown in Figures 9a-b and 10. In each case there is an S-shape to the probability plot and the pvalues of the tests all reject the null hypothesis that the distribution is normal.

Gregoire and Reynolds (1988) presents the test statistic

$$
T=\frac{\bar{D}}{S_{\bar{D}}}
$$


to test the null hypothesis $\mathrm{H}_{0}: \mu=0$, where $S_{\bar{D}}$ is the standard error of $\bar{D}$ which is the mean of the paired differences. This is a restatement of a paired $t$ test (Ott 1993) where the mean difference is expected to be zero. Table 4 shows the paired $t$ values that were calculated for each of the groups. Only distances of 6, 9, and 12 meters did not reject the null hypothesis that the difference between the camera and caliper means equals zero with $\alpha=0.05$. Though this test also assumes normality, it is less sensitive (given a large sample size) to the shape of the distribution.

As an alternative, the Wilcoxon signed-rank test (Ott 1993) was applied to test whether or not the distribution of the differences is symmetric around zero. This test is recommended when the distribution has "heavy tails"(more than expected number of observations with extreme deviations from the mean). Minitab was used to run this analysis and it did lead to different results. Using this test, only the 6 meter distance did not reject the null hypothesis at the $95 \%$ confidence level (p-value 0.083). The fact that the distributions are not normal is unfortunate since it hinders the types of analysis that can be performed on these data.

Ignoring the normal distribution assumption and calculating the maximum anticipated error (Equation 6) gives the results found in Table 4. Actual ranges found by ordering the data from smallest to largest taking the sign into account and examining the actual observations obtained using equation 7 are shown by the $95 \%$ range in Table 4.

$$
o b s_{U \& L}=\left(\frac{n_{t}}{2}\right) \pm\left(\frac{(1-\alpha) n_{t}}{2}\right)
$$

where

$$
\begin{aligned}
& o b s_{U \& L}=\text { upper and lower ordered observations representing the 1- } \alpha \text { percent } \\
& \text { confidence level } \\
& n_{t}=\text { sample size of group } t
\end{aligned}
$$


We can see from these results that Freese's chi-square test still gives a reasonable approximation for this sample despite the divergence of the distribution from normality.

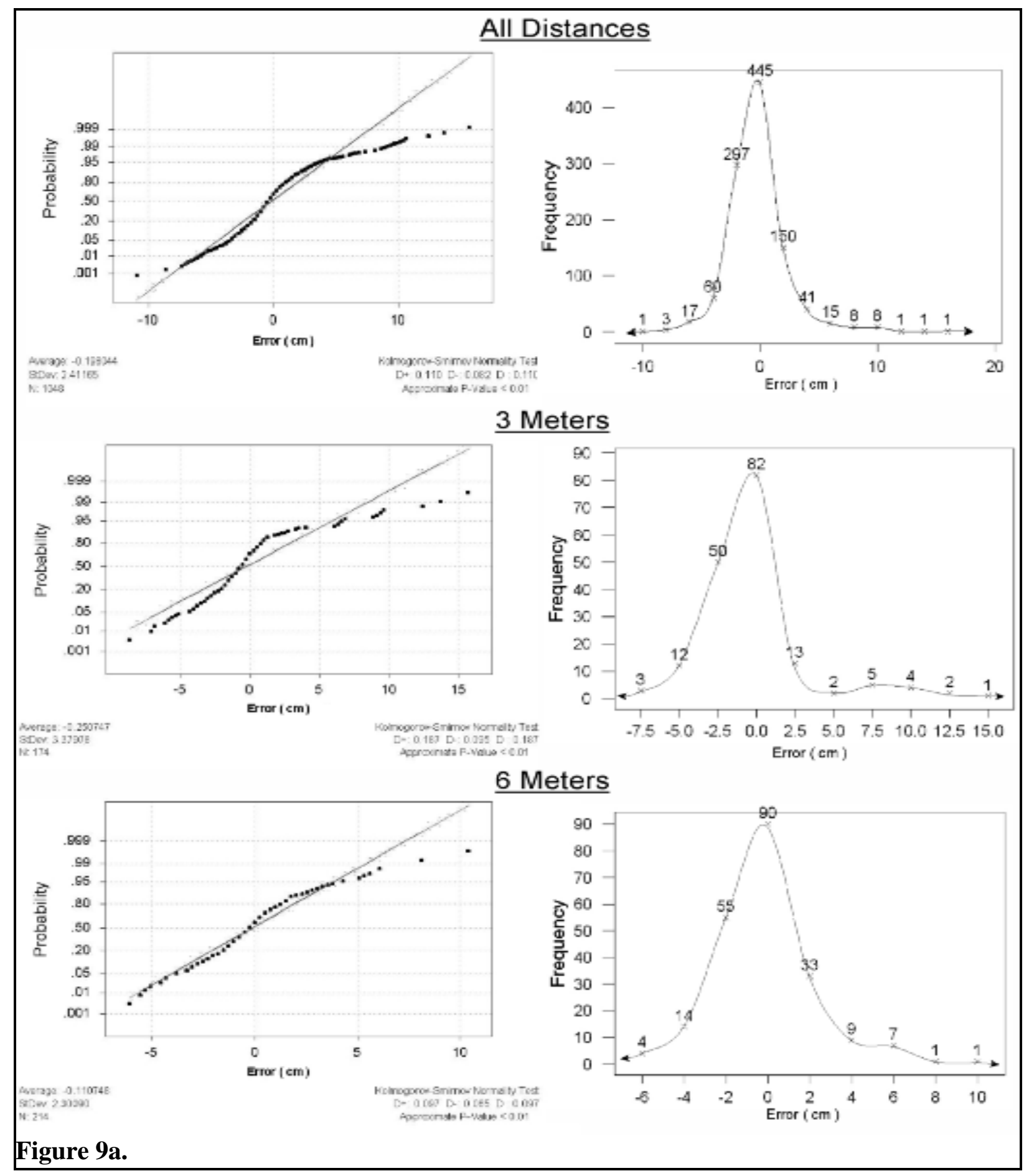




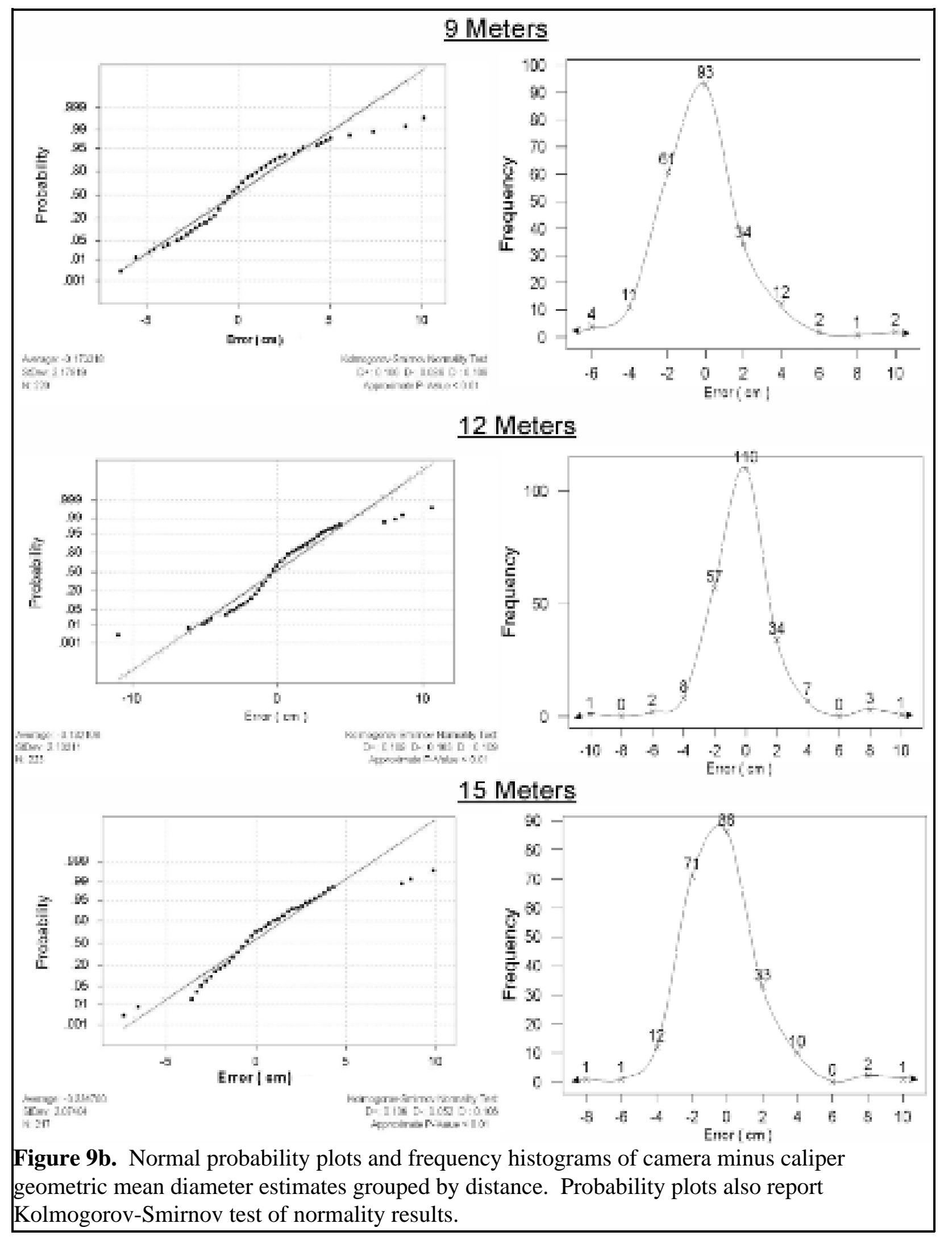




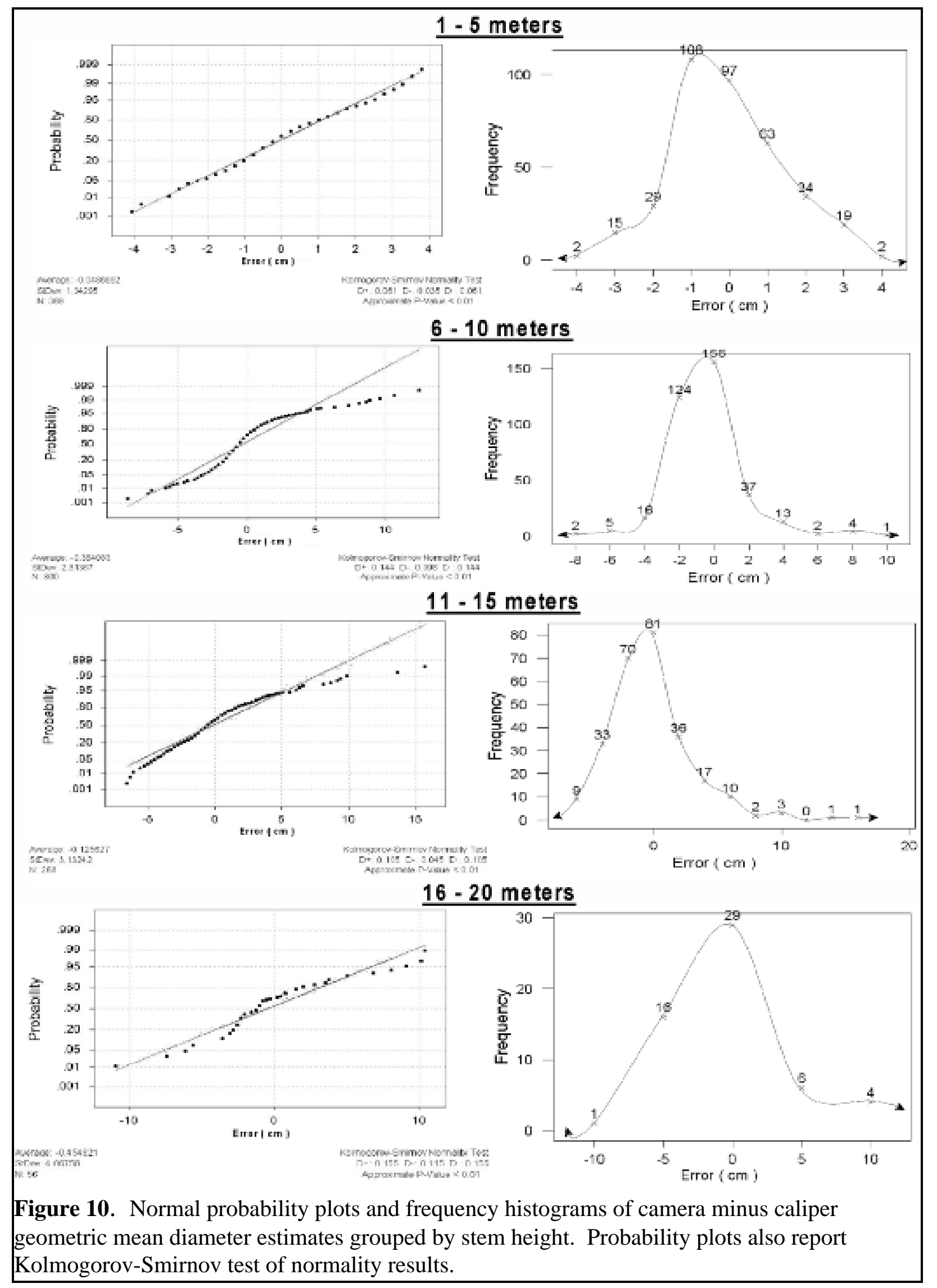




\begin{tabular}{|c|c|c|c|c|c|c|}
\hline & & & Distance & & & \\
\hline & 3 meter & 6 meter & 9 meter & 12 meter & 15 meter & all \\
\hline Mean $(\mathrm{cm})$ & -0.458 & -0.111 & -0.173 & -0.132 & -0.335 & -0.232 \\
\hline Std. Dev. $(\mathrm{cm})$ & 3.039 & 2.301 & 2.178 & 2.133 & 2.074 & 2.337 \\
\hline $\begin{array}{r}\text { Median }(\mathrm{cm}) \\
\text { Mode(cm) }\end{array}$ & $\begin{array}{c}-0.762 \\
0\end{array}$ & $\begin{array}{c}-0.254 \\
0\end{array}$ & $\begin{array}{l}-0.508 \\
-1.016\end{array}$ & $\begin{array}{c}-0.254 \\
0\end{array}$ & $\begin{array}{l}-0.508 \\
-0.762\end{array}$ & $\begin{array}{l}-0.508 \\
0\end{array}$ \\
\hline paired t value & -1.987 & -0.701 & -1.180 & -0.926 & -2.374 & -3.217 \\
\hline $\begin{array}{c}E(\mathrm{~cm}) \\
95 \% \text { range }(\mathrm{cm})\end{array}$ & $\begin{array}{c}5.5 \\
(-5.8,6.8)\end{array}$ & $\begin{array}{c}4.2 \\
(-4.5,4.8)\end{array}$ & $\begin{array}{c}4.0 \\
(-4.6,4.8)\end{array}$ & $\begin{array}{c}3.9 \\
(-3.6,3.8)\end{array}$ & $\begin{array}{c}3.8 \\
(-3.3,3.8)\end{array}$ & $\begin{array}{c}4.7 \\
(-4.6,5.3)\end{array}$ \\
\hline & & & Height & & & \\
\hline & $1-5 \mathrm{~m}$ & $6-10 m$ & $11-15 m$ & $16-20 \mathrm{~m}$ & & \\
\hline $\mathrm{n}$ & 369 & 360 & 263 & 56 & & \\
\hline Mean $(\mathrm{cm})$ & -0.053 & -0.459 & -0.126 & -0.458 & & \\
\hline Std. Dev. $(\mathrm{cm})$ & 1.346 & 2.07 & 3.134 & 4.058 & & \\
\hline Median $(\mathrm{cm})$ & -0.25 & -0.51 & -0.51 & -1.02 & & \\
\hline Mode(cm) & 0.00 & 0.00 & -1.02 & -1.02 & & \\
\hline paired $t$ value & -0.755 & -4.207 & -0.652 & -0.844 & & \\
\hline $\mathrm{E}(\mathrm{cm})$ & 2.5 & 4.3 & 5.7 & 7.0 & & \\
\hline $95 \%$ range $(\mathrm{cm})$ & $(-2.8,2.8)$ & $(-4.3,4.3)$ & $(-5.1,6.7)$ & $(-7.4,10.2)$ & & \\
\hline
\end{tabular}

\subsection{Analysis of Variance Results}

\subsubsection{General Information}

Analysis of variance was performed on the data to examine the effects of distance and height when selecting the best data collection protocol. The analyses were run using the SAS pr oc mi xed function (Appendix C). The 20 stems were classified into diameters (breast height) with five stems each falling into the four classes - $(16-30 \mathrm{~cm}),(31-45 \mathrm{~cm}),(46-60$ $\mathrm{cm})$, and (> $60 \mathrm{~cm})$. The distances of 3, 6, 9, 12, and 15 meters were used as treatments and height in 1.2 meter increments (16 levels, beginning at 1.4 meters from the ground) was a 
blocking variable. Heights were later classified into four strata. Essentially only distances could be considered treatments because that is the only factor that we can control. Diameter and height are blocking factors for the purposes of observing their effects within the various treatments.

Because every stem was an experimental unit for each of the treatments, repeated measures tests were used to account for the effects of the subjects (stems). Unfortunately, unequal sample sizes precluded our planned use of Fisher's LSD (least significant difference) for multiple comparisons. Instead, orthogonal contrasts were examined for differences among the means. Several models were run to determine the significant effects that contributed to error using this camera technique. Distance, diameter, height, and distance/height interaction were used as fixed effects in the models. There were no random effects. All of the effects were used in the first model. All subsequent models examined each effect separately.

\subsubsection{All-Inclusive Model}

The first model that was run included all of the factors. Only height and the interaction term showed significant effects at the $95 \%$ confidence level. Least squares means of the heights reveal that diameter estimates obtained at three heights $(1.4,11$, and $14.6 \mathrm{~m})$ were significantly different from the expected mean of zero. Two of these means were greater than zero and the 11 meter mean was less than zero. Least squares means were not calculated for the 18.3 and 19.5 meter heights due to lack of observations among some of the treatment groups. In a model using only height as a fixed effect only the 1.4 meter height remains significantly different from zero, with approximately the same least squares mean and standard deviation. The 11 meter least squares mean changes by $0.04 \mathrm{~cm}$ and the p-value changes from 0.04 to 0.06 , thus from 


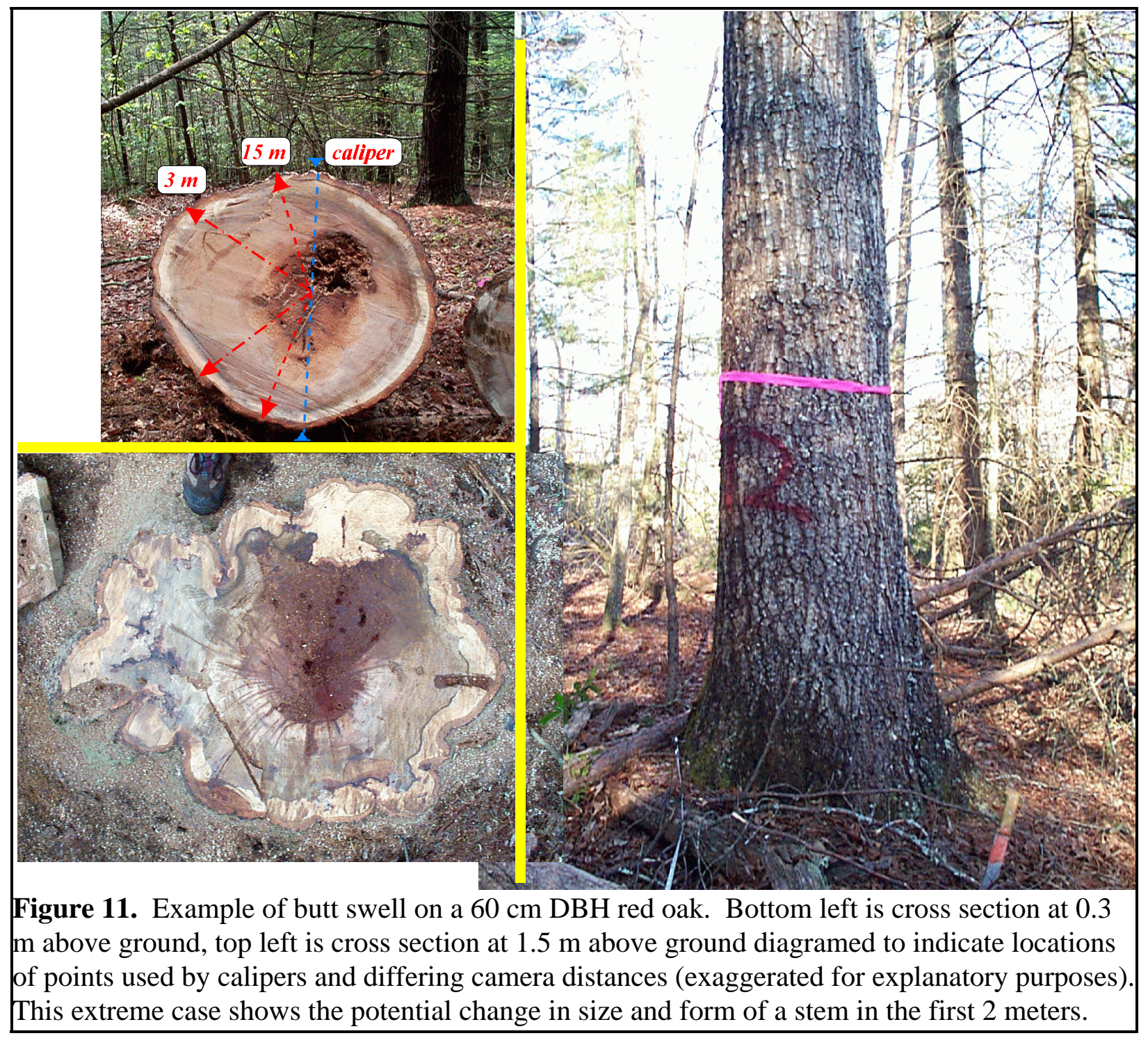

marginally significant to marginally non-significant at the $95 \%$ level. The 14.6 meter least squares mean changes drastically however, from 0.77 to $-0.03 \mathrm{~cm}$. The greater height measurements $(>16 \mathrm{~m}$ ) should be interpreted keeping in mind that greater than half of the possible measurements could not be collected.

Looking closer at the individual observations that contributed to these anomalies helped to offer an explanation of their causes. Out of the heights that were included in this study, the 1.4 meter height is the one most affected by butt swell. This swell is evidenced by severe taper in the 
lower portion (e.g. 0 - 2 meter height) of the bole (Figure 11). Thus there is the possibility of a systematic discrepancy between the location of this height using the tape or the camera. Two stems in particular showed a rather consistent positive bias between 1 to $3 \mathrm{~cm}$ for all distances. There are different explanations for discrepancy based on distance. Most of the base points at the closer distances were located at the extreme edges of the image plane in order to maximize the amount of stem that could be captured on the image plane. This may have caused incorrect location of the 1.2 meter measurement, which was often the only height measurement on the base images taken at 3 meters distance. Camera station distance is inversely proportional to the distance between the points that represent the diameter for that image and the points measured using the caliper (Figure 11), assuming the caliper measured points are in a plane passing through the stem axis perpendicular to the camera axis. Assuming that orientation to the "major-axis" of the cross section is random, this should not be that severe. The points that represent the diameter are also located farther from the principal point on the image, which increases the likelihood of any interior orientation or lens effects. Another possible explanation for a positive bias at the shortest distance is an incorrect focal length used in the calculations. The camera used in this study disallowed locking the lens at a specified focal length. It is possible that the focal length was adjusted at this distance.

At father distances from the stem other factors are of primary concern. At this point the object distance is effectively infinite within a certain tolerance. The major problem with the lower portion of the stem at this distance is the lower reflectance received at the image plane. There is greater difficulty distinguishing the edges of the stem at this distance due to foreground interference as well as lower contrast caused by background reflectance which also tends to pull the edges out (Figure 12). 

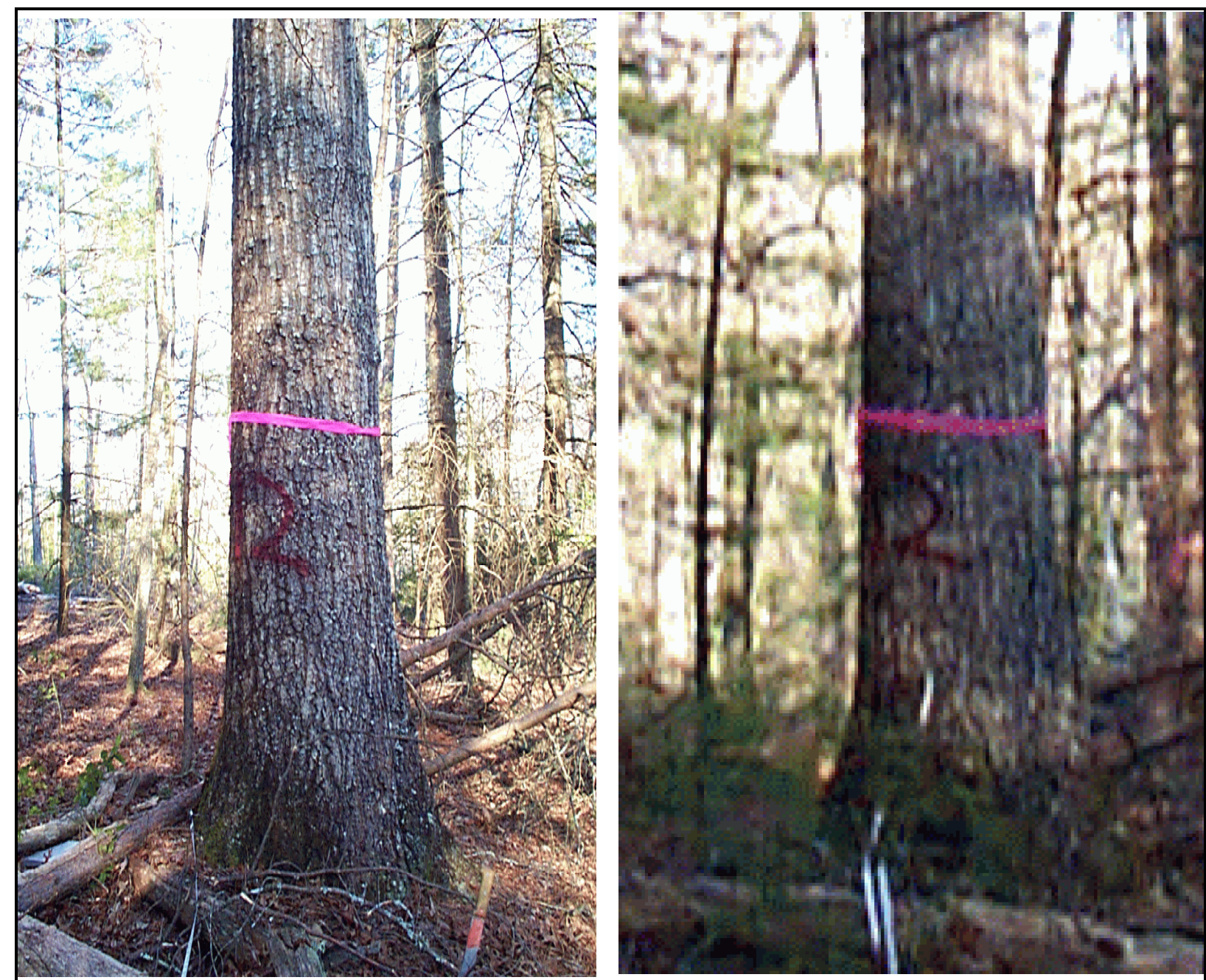

Figure 12. Lower section of stem at the 3 and the 15 meter camera stations (left to right). Pixel resolution at the edge of the stem at the flagging is 2.2 and 11 millimeters for the 3 and 15 meter distances, respectively.

\subsubsection{Height Model}

Over-stratification may have been the causal factor of the significant effect in the all-

inclusive model. To circumvent this problem a coarser stratification was applied. The data were grouped into four classes - (1-5 m), (6-10 m), (11-15 m), and $(16-20 \mathrm{~m})$. Using only these heights as effects in the model did not show any significant diameter biases. Table 4 shows that the standard deviations of these observations increase about $1 \mathrm{~cm}$ for every $5 \mathrm{~m}$ increase in height. A transformation of the data by some function of height could be used to normalize the 
variance, but interpretation of the results would be problematic.

This variance difference is readily apparent in the scatter plots of the errors by height at various distances (Figure 13). Visual interpretation of these plots shows that in every case the variance increases with height. The errors at the 3 meter distance are more dispersed than any other distance. The $95 \%$ ranges derived from the ordered differences bring the magnitude of these results to light (Table 4). There also appears to be a negative trend in error with increased height. This is supported by the decrease of median values with increasing heights (Table 4).

\subsubsection{Distance Model}

The model using only the distance as the only effect did not show any significant differences at the $5 \%$ level. It can be noted however that the average deviation from zero at the 3 meter distance was greater than any other distance (Table 4). The largest standard deviation was also at the 3 meter distance.

\subsubsection{Diameter Model}

The diameter only model failed to reject the null hypothesis that the means were the same for each diameter class. The least squares means were $-0.48,-0.03,0.02$, and $-0.02 \mathrm{~cm}$ for the $16-30 \mathrm{~cm}, 31-45 \mathrm{~cm}, 46-60 \mathrm{~cm}$, and $>60 \mathrm{~cm}$ classes, respectively. Because the entire stem was included in these categories rather than just measurements that fell within each of these classes the interpretation of this test would say more about the morphology of stems in these classes than about the effectiveness of the instrument in measuring differing diameters. We could compare the measurements that fell within these classes, but sample sizes would vary greatly among the groups for obvious reasons. There is also an issue of covariance in that larger measurements occur at lower heights on the bole.

There were 35 diameter estimates $>60 \mathrm{~cm}$ as measured by the calipers with errors 


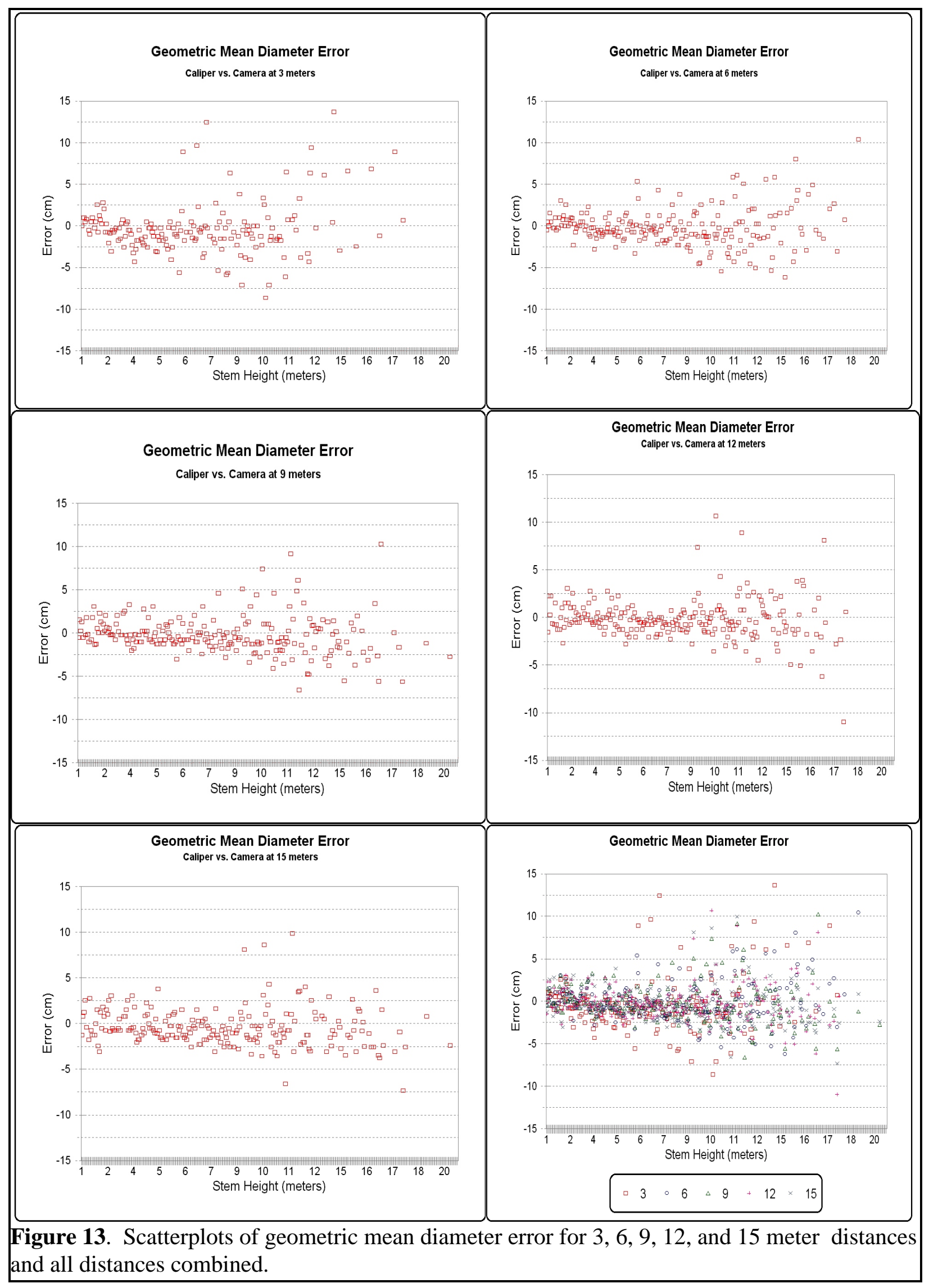


ranging from -3.0 to $3.2 \mathrm{~cm}$ with an arithmetic mean of $-0.17 \mathrm{~cm}$ and standard deviation \pm 1.63

$\mathrm{cm}$. These numbers are not quite as good as the $1-6$ meter measurements overall (where most of the $>60 \mathrm{~cm}$ measurements occurred), not taking sample size in to account. Suppostionally, size should have no effect. This disregards any increase in proportional deviation from the assumed circular cross section as well as any effects from longer image distances or image distances farther from the principal point.

\subsection{Volume Estimation}

The strength of using the camera as opposed to other instruments lies in the amount of data that it is able to collect in a short period of time. For single diameter measurements there are assuredly other instruments that are as capable and more efficient. Volume estimation is one of the proposed advantages of this tool. Estimation of the volume of a stem can be approximated from a single diameter and length measurement used in a simple geometric model or within an empirical equation already established. The more data input into the model the more reliable it will be provided that the input data is accurate. Hence a model that uses diameters at five heights should be more reliable than a model using only two diameters. There is a trade off at a certain point where the cost of collecting the data will surpass the benefit gained from having a more reliable estimate.

Volumes were calculated as described in section 3.6. Volumes were calculated for each of the 20 stems at each of the distances, regardless of missing camera observations. In the case where there were missing field observations, only the section of the stem measured in the field was compared. For example, if a diameter at 1.4 meters was not recorded, only the stem section from 2.6 meters to the measured top was evaluated for both camera and caliper. For the stem that split when felled, single caliper measurements were used up to 9.8 meters. The GMDs were 
used when possible for the camera estimates, but directional diameters were used where the GMDs could not be calculated.

Again a repeated measures analysis of variance model was used in SAS. The measurement variables were volume differences in cubic meters and percent. Only distance was used as a factor because height is intrinsically related to volume and diameter was not found to have any significant effect. The test of the null hypothesis that the means for all of the distances were the same was marginally not rejected at the $95 \%$ confidence level (p-value 0.0576 ). Orthogonal contrasts, comparing each distance to the other four, showed only the 3 meter distance to be significantly different for volume in cubic meters and percent. The least squares mean deviations were -0.153 cubic meters or $-9.6 \%$ for the 3 meter distance. The least squares means for the other distances ranged from -0.025 to 0.017 cubic meters or -2.8 to -0.1 percent.

Table 5 shows the means, standard deviations, and maximum anticipated errors (E) by distance as well as diameter. The 12 meter distance resulted in the mean closest to zero and had the lowest standard deviation though it is not significantly different from other distances in the 6 to 15 meter range. The maximum anticipated errors and standard deviations in cubic meters are lowest for the smallest diameter. This is not surprising because this error should be proportional to size. Lack of significant differences among the maximum anticipated errors in percent support this assumption.

\subsubsection{Volume Estimation Comparison}

In order to better see the advantages of this system the results were compared to results from an alternative method. The alternative method was using volume equations developed specifically for northern red oak in the southern Appalachian mountains. The equations used were for cubic foot volume (Equations 8 and 9), later converted to cubic meters, for wood and 
Table 5. Summary statistics for volume estimates by distance and diameter for 20 red oak stems. Reported are the arithmetic means, standard deviations, and the maximum anticipated error (E) using the chi-square test for accuracy.

\begin{tabular}{|c|c|c|c|c|c|c|}
\hline & \multirow[b]{2}{*}{3 meter } & \multicolumn{4}{|c|}{ Nominal Distance } & \multirow[b]{2}{*}{ all } \\
\hline & & 6 meter & 9 meter & 12 meter & 15 mete & \\
\hline \multicolumn{7}{|c|}{ cubic meters } \\
\hline Mean & -0.170 & -0.042 & -0.023 & 0.000 & -0.032 & -0.053 \\
\hline Std. Dev. & \multirow{2}{*}{$\begin{array}{l}0.320 \\
0.558\end{array}$} & 0.145 & 0.141 & 0.140 & \multirow{2}{*}{$\begin{array}{l}0.155 \\
0.242\end{array}$} & 0.199 \\
\hline \multirow[t]{2}{*}{$E$} & & 0.232 & 0.219 & 0.215 & & 0.361 \\
\hline & \multirow[b]{2}{*}{-10.4} & & percent & & & \\
\hline Mean & & -3.7 & -2.3 & -1.1 & \multirow{2}{*}{$\begin{array}{l}-3.4 \\
8.1\end{array}$} & -4.2 \\
\hline Std. Dev. & \multirow{2}{*}{$\begin{array}{l}15.3 \\
28.5\end{array}$} & 7.6 & 6.6 & 6.6 & & 9.8 \\
\hline $\mathrm{E}$ & & 13.1 & 10.8 & 10.3 & $\begin{array}{c}8.1 \\
13.5\end{array}$ & 18.7 \\
\hline \multirow{2}{*}{ 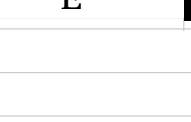 } & \multirow{2}{*}{\multicolumn{5}{|c|}{$\begin{array}{l}\text { Nominal Diameter } \\
31-45 \mathrm{~cm}\end{array}$}} & \\
\hline & & & & & & $>60 \mathrm{~cm}$ \\
\hline \multirow[t]{2}{*}{$\mathrm{n}$} & \multicolumn{2}{|c|}{25} & 25 & \multirow{2}{*}{\multicolumn{2}{|c|}{25}} & 25 \\
\hline & & \multirow[b]{2}{*}{-0.021} & cubic meters & & & \\
\hline \multicolumn{2}{|l|}{ Mean } & & -0.043 & \multicolumn{2}{|c|}{-0.090} & -0.059 \\
\hline \multirow{3}{*}{$\begin{array}{l}\text { Std. Dev. } \\
\text { E }\end{array}$} & \multicolumn{2}{|c|}{0.022} & 0.120 & \multicolumn{2}{|c|}{0.281} & 0.258 \\
\hline & \multicolumn{2}{|c|}{0.048} & 0.201 & \multicolumn{2}{|c|}{0.465} & 0.416 \\
\hline & & & percent & & & \\
\hline Mean & \multicolumn{2}{|c|}{-7.1} & -4.2 & \multicolumn{2}{|c|}{-3.7} & -1.6 \\
\hline \multirow{2}{*}{$\begin{array}{l}\text { Std. Dev. } \\
F\end{array}$} & \multirow{2}{*}{\multicolumn{2}{|c|}{$\begin{array}{r}6.1 \\
14.9\end{array}$}} & 10.2 & \multirow{2}{*}{\multicolumn{2}{|c|}{$\begin{array}{l}12.9 \\
20.7\end{array}$}} & 8.8 \\
\hline & & & 17.4 & & & 14.3 \\
\hline
\end{tabular}

bark using DBH and height to a 4-inch top as independent variables (Clark and Schroeder 1986).

This equation still requires an instrument to take upper stem measurements in order to obtain an estimate with any precision. For the comparison in this study the GMDs from the caliper estimates were used along with the field taped heights for equation input parameters. Because these are the same measurements used in "actual" volume calculation, any error comes from the model. Height to a four-inch top was not acquired in the field for every tree. Diameter data was only collected for some trees up to the end of the merchantable stem. This is seen particularly in the larger diameter stems where the equations produce an underestimate of volume. For many of these stems without a clear dominant main stem, it is often difficult to determine which four-inch top should be measured. 


$$
\begin{gathered}
\text { volume }_{<11^{\prime \prime}}=0.03592\left(d^{2} h\right)^{0.73586} \\
\text { volume }_{>11^{\prime \prime}}=0.01199\left(d^{2}\right)^{0.95561} h^{0.73586}
\end{gathered}
$$

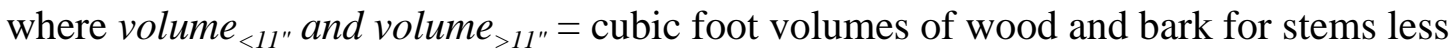
than 11 inches and greater than 11 inches, respectively.

$d=$ diameter at breast height in inches

$h=$ height to a four-inch top in feet

Table 6. Volume estimates for 20 red oak stems comparing camera estimates from the 12 meter distance $(12 \mathrm{~m})$, the average of all camera estimates (avg-cal), and the equation estimates (equ-cal) to the "true" caliper estimates. The left four columns show the stem volumes in cubic meters, the next three columns from the left show the cubic meter differences, and the final

\begin{tabular}{|c|c|c|c|c|c|c|c|c|c|c|}
\hline & \multicolumn{3}{|c|}{ cubic meters } & & \multicolumn{3}{|c|}{ cubic meters } & \multicolumn{3}{|c|}{ percent } \\
\hline & caliper & $12 \mathrm{~m}$ & AVG & equation & 12-cal & avg-cal & equ-cal & 12-cal & avg-cal & equ-cal \\
\hline 1 & 0.80 & 0.77 & 0.74 & 0.77 & -0.03 & -0.06 & -0.03 & -3.6 & -8.0 & -3.6 \\
\hline 2 & 0.78 & 0.79 & 0.77 & 0.70 & 0.01 & -0.01 & -0.08 & 1.5 & -1.0 & -9.9 \\
\hline 3 & 0.29 & 0.27 & 0.27 & 0.33 & -0.02 & -0.01 & 0.05 & -7.2 & -4.7 & 15.8 \\
\hline 4 & 2.44 & 2.53 & 2.32 & 1.85 & 0.09 & -0.12 & -0.59 & 3.7 & -4.9 & -24.1 \\
\hline 5 & 3.74 & 3.61 & 3.50 & 2.72 & -0.13 & -0.24 & -1.01 & -3.4 & -6.3 & -27.2 \\
\hline 6 & 0.76 & 0.75 & 0.76 & 1.09 & -0.02 & 0.00 & 0.32 & -2.0 & 0.1 & 42.3 \\
\hline 7 & 1.60 & 1.55 & 1.55 & 1.75 & -0.05 & -0.04 & 0.16 & -2.8 & -2.5 & 9.9 \\
\hline 8 & 0.17 & 0.18 & 0.16 & 0.20 & 0.00 & -0.01 & 0.03 & 1.7 & -6.4 & 17.0 \\
\hline 9 & 0.36 & 0.32 & 0.32 & 0.43 & -0.03 & -0.03 & 0.07 & -8.6 & -9.7 & 20.4 \\
\hline 10 & 0.55 & 0.51 & 0.52 & 0.56 & -0.04 & -0.03 & 0.01 & -7.8 & -5.4 & 1.3 \\
\hline 11 & 3.45 & 3.75 & 3.70 & 3.15 & 0.30 & 0.25 & -0.29 & 8.8 & 7.3 & -8.5 \\
\hline 12 & 2.70 & 2.72 & 2.25 & 2.21 & 0.02 & -0.44 & -0.49 & 0.9 & -16.5 & -18.0 \\
\hline 13 & 1.40 & 1.21 & 1.18 & 1.30 & -0.18 & -0.22 & -0.10 & -13.2 & -15.4 & -7.0 \\
\hline 14 & 4.84 & 4.48 & 4.62 & 3.69 & -0.37 & -0.22 & -1.15 & -7.5 & -4.5 & -23.7 \\
\hline 15 & 1.42 & 1.36 & 1.45 & 1.41 & -0.06 & 0.03 & -0.01 & -4.1 & 2.4 & -0.7 \\
\hline 16 & 3.26 & 3.51 & 3.37 & 2.81 & 0.25 & 0.10 & -0.46 & 7.5 & 3.2 & -14.0 \\
\hline 17 & 2.39 & 2.40 & 2.29 & 1.91 & 0.00 & -0.10 & -0.49 & 0.1 & -4.1 & -20.4 \\
\hline 18 & 0.51 & 0.54 & 0.50 & 0.52 & 0.02 & -0.01 & 0.01 & 4.9 & -2.8 & 1.3 \\
\hline 19 & 1.13 & 1.23 & 1.16 & 1.05 & 0.10 & 0.03 & -0.08 & 8.5 & 2.3 & -7.3 \\
\hline 20 & 2.51 & 2.69 & 2.62 & 2.21 & 0.17 & 0.10 & -0.30 & 6.9 & 4.1 & -12.0 \\
\hline Mean & 1.75 & 1.76 & 1.70 & 1.53 & 0.00 & -0.05 & -0.22 & -0.79 & -3.65 & -3.42 \\
\hline Std. Dey & 1.34 & 1.34 & 1.32 & 1.02 & 0.14 & 0.15 & 0.38 & 6.31 & 6.08 & 17.56 \\
\hline
\end{tabular}
three columns report the percent differences.

There can be several observations drawn from the data in Table 6. Firstly, the equation provides a reasonably accurate assessment of volume for the reduced amount of work and man hours required. The cubic meter estimates for the equation and the camera average of all distances is less than the "actual", with the equation estimates resulting in the most negative bias. 
The standard deviations of the cubic meter differences are at least twice as large for the equation estimates than for the camera estimates. The explanation for this is that the camera and caliper estimates are of the same sample, whereas the equation estimates are derived from volumes expected within a larger sample region.

The percent differences show this standard deviation discrepancy as well. The percent difference numbers should be interpreted with caution since equal cubic foot errors can result in drastically different percent errors varying with stem size.

Site characteristics can have a notable effect on application of volume tables or equations. Twelve of the stems collected in this study were from an area on the Pisgah National Forest in southwestern North Carolina which is the location of some of the sample stems used in creating the volume equation. The remaining eight stems were located in the ridge and valley region of Virginia. The North Carolina stems were all on flat to moderately sloped terrain that appeared to be well drained. There was one stem in the smallest diameter class that was growing in a floodplain area. The Virginia stems were all located on a ridge top with very shallow, welldrained soil.

\begin{tabular}{|c|c|c|c|c|c|c|c|}
\hline & \multicolumn{3}{|c|}{ cubic meters } & & & \multicolumn{2}{|l|}{ percent } \\
\hline & $12 \mathrm{~m}$ & AVG & equation & & $12 \mathrm{~m}$ & $\overline{A V G}$ & equation \\
\hline & & & \multicolumn{3}{|c|}{ North Carolina } & & \\
\hline Mean & 0.02 & -0.04 & -0.27 & & -0.31 & -3.27 & -7.40 \\
\hline \multirow[t]{2}{*}{ Std. Dev. } & 0.18 & 0.18 & 0.34 & & 7.74 & 7.64 & 12.00 \\
\hline & & & & Virginia & & & \\
\hline Mean & -0.02 & -0.06 & -0.14 & & -1.52 & -4.22 & 2.54 \\
\hline Std. Dev. & 0.06 & 0.08 & 0.44 & & 3.58 & 2.81 & 23.30 \\
\hline
\end{tabular}


The mean errors for the camera estimates did not vary widely between locations, whereas the equation underestimation bias of the North Carolina sample is nearly double that of the Virginia sample (Table 7). The standard deviations of the camera estimates were two to three times as large on the North Carolina stems as on the Virginia stems. The equation estimates had slightly larger standard deviations on the Virginia stems than the North Carolina stems. The standard deviations for the differences in both cubic meters and percent were 1.5 to 6 times larger for the equation estimates than for the camera estimates. This supports the fact that selection of volume tables or equations is crucial to the reliability of the output. Reliability of estimation is directly proportional to ability in matching the range (e.g., location, species, diameter classification resolution, form information, etc.) and resolution (e.g., region level data to stand level data, intensity of data input in model formulation) of the model used for estimation to the actual phenomena being estimated.

\subsection{Sources of Errors}

There are many factors that contribute to error using the methods described in this paper. There is the error of collecting and recording incorrect field data assumed to be "true." Errors inherent in this type include the following: error in the measurement of field reference data; mislocation of points used for diameter determination because of height, line of sight, and tangential differences; and various camera related sources of error.

\subsubsection{Mislocation Error}

Unlike similar studies which test the capabilities of an instrument rather than the reliability of a total method, the heights of the diameters were not marked but were determined by the camera system and the conventional system independently. While not the best way to make comparisons to other methods, it provides more realistic results of expected 


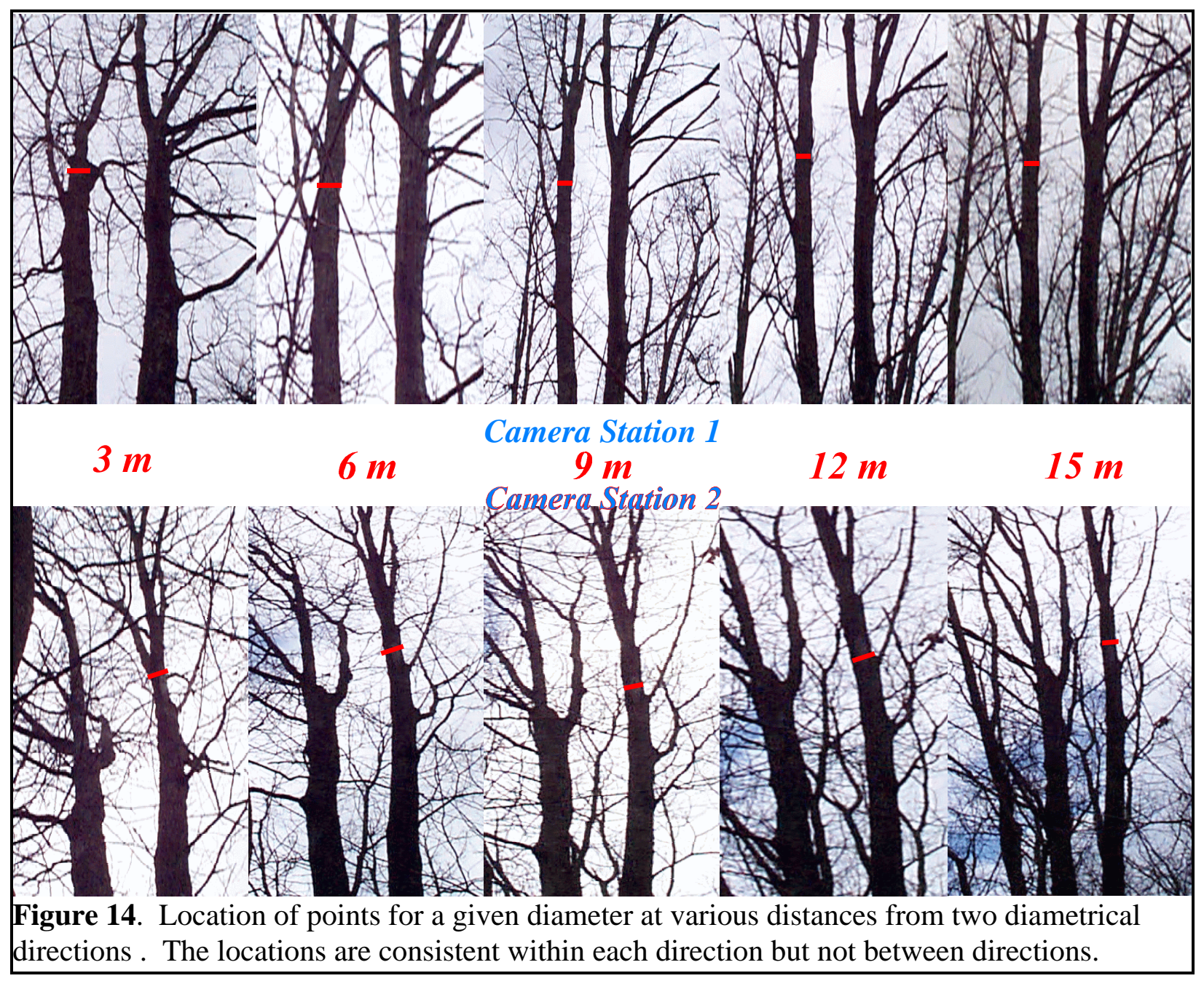

performance. The hypsometric component of a dendrometer is an extremely important factor of its usefulness. Figure 14 shows the location of a diameter measurement at the different distances for two opposing camera stations. The location of the diameters is fairly consistent by direction, but differs between the two camera stations. Using the branch as a marker it is apparent that the points measured from the camera station 2 direction are higher on the stem than the points measured from the diametrical direction. This is primarily a result of lean which is not accounted for using the methods set forth and used in this study. Measurement of inclination angle and camera factors can also contribute to this mislocation, but to a lesser extent.

It should also be noted that both bole portions in each frame of Figure 14 are on the 
same stem. This poses problems with the method presented in this paper. If the camera station orientations are orthogonal to the fork as in Figure 15, overlap, as well as lean, becomes a problem when attempting to distinguish the edge points.

Within the selected height plane there are two other mislocation problems. One is the difference in the line of sight around the stem axis between the camera station and the direction measured by the calipers. The second problem is that of measuring points on the circumference that are farther from the caliper measured points with decreasing camera station distance (Figures 9a and 9b, pages 29-30).

\subsubsection{Camera Related Errors}

Interior orientation and the precision

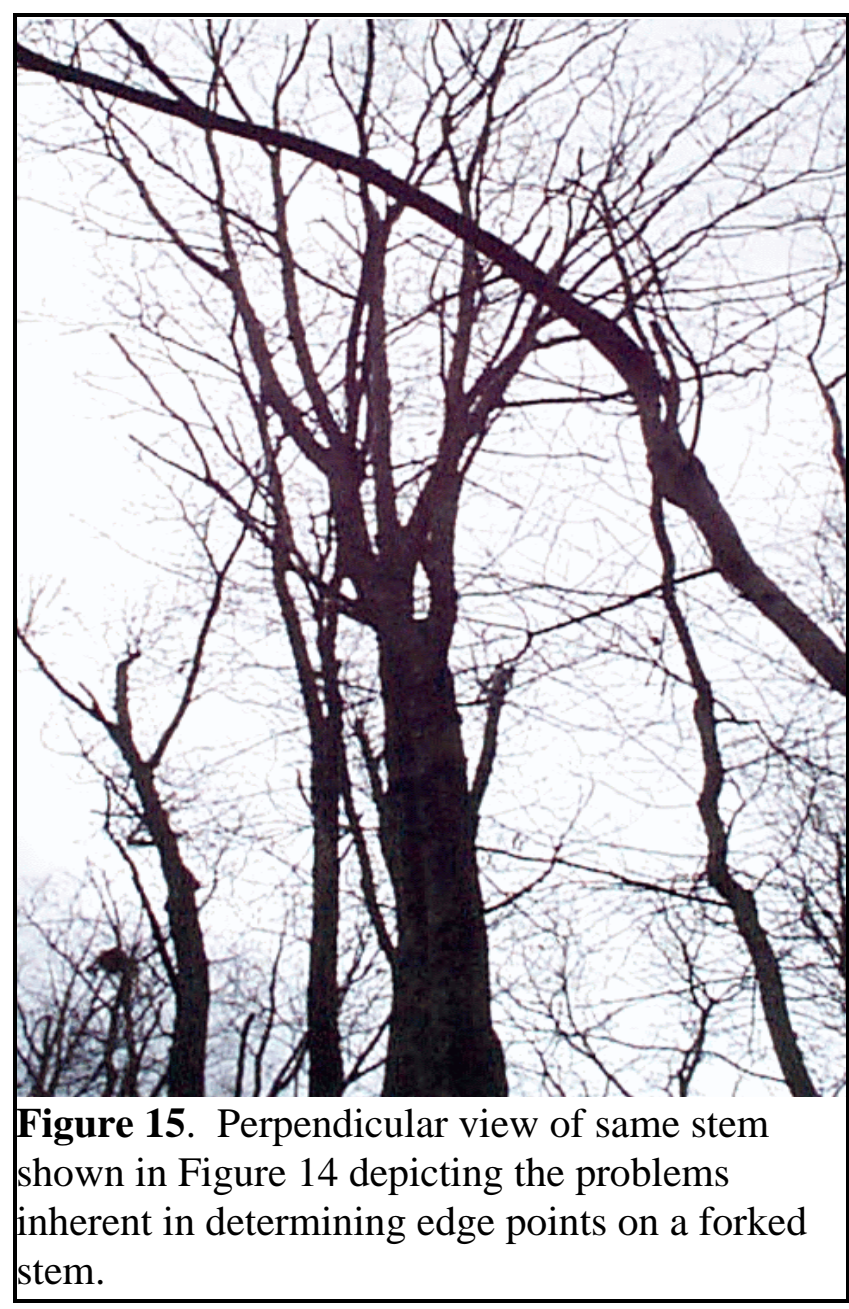

of the CCD are sources of error introduced by the camera. Interior orientation is a term that describes the "parameters that model the passage of light rays through the lens and onto the image plane" (Fryer 1989). Lens distortion, image plane deformation, and the orientation of the CCD array to the focal point are included in this definition.

$$
\text { Lens distortions are caused by }
$$

aberrations in the manufacture of the lens that refract light in a manner not predicted. Usually lens distortions are broken down into radial and tangential components. 
These components are assumed to be systematic and can be corrected for, though this was not done in this study. Radial lens distortion is often significant near the edges of the image.

Image plane deformation would occur if any of the $\mathrm{CCD}$ elements were out of alignment. This would likely only have a limited effect at a certain location (row and column) in the image, and should be inconsequential.

Lastly the CCD array itself could be tilted or misaligned in relation to an axial ray coming directly through the center of the lens. Ideally this ray should pass through the principal point on the image plane. This could perhaps explain some of the negative bias in the upper stem heights resulting from the correlation of these heights with image space location. Considering the small size of the CCD array and the short focal length a small amount of tilt could have substantial

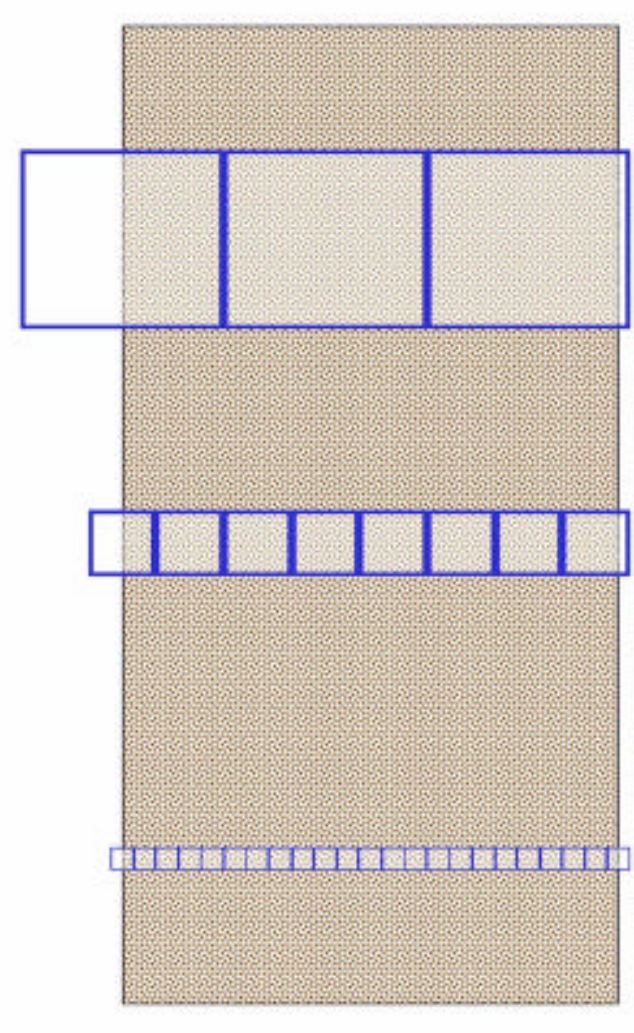

Figure 16. Diagram showing error increase due to loss of precision.

( effects. An added complication is that the nonmetric nature of the camera may cause this effect to be random rather than systematic, making corrections impossible.

Though the consequences of some of these camera parameters are unknown, the precision issue (Figure 16) has a definite effect. Precision of pentaprism calipers is regulated by the gradations on the instrument. In a similar way, the precision of rangefinder dendrometers is constrained by the precision of angular measurement. This also is the case with the 
camera. Thus, overall precision is directly related to object space distance from the measurement points on the stem to the camera. This partially explains increased variance at upper stem heights and, theoretically, with increased distances to the tree; although lean effects overshadowed the precision issue with the methods used in this study.

\subsubsection{Procedural Errors}

Procedural errors occur from data capture to information output. These can be errors in the measurement or transfer of ancillary data, image capture, image measurement, and calculation. These errors vary in magnitude and frequency of occurrence. One surety is that increasing complexity leads to increased error potential.

Camera station set-up and ancillary data collection errors can occur at any stage in the process. Distance measurement with the nylon tape is hampered by underbrush that keeps the

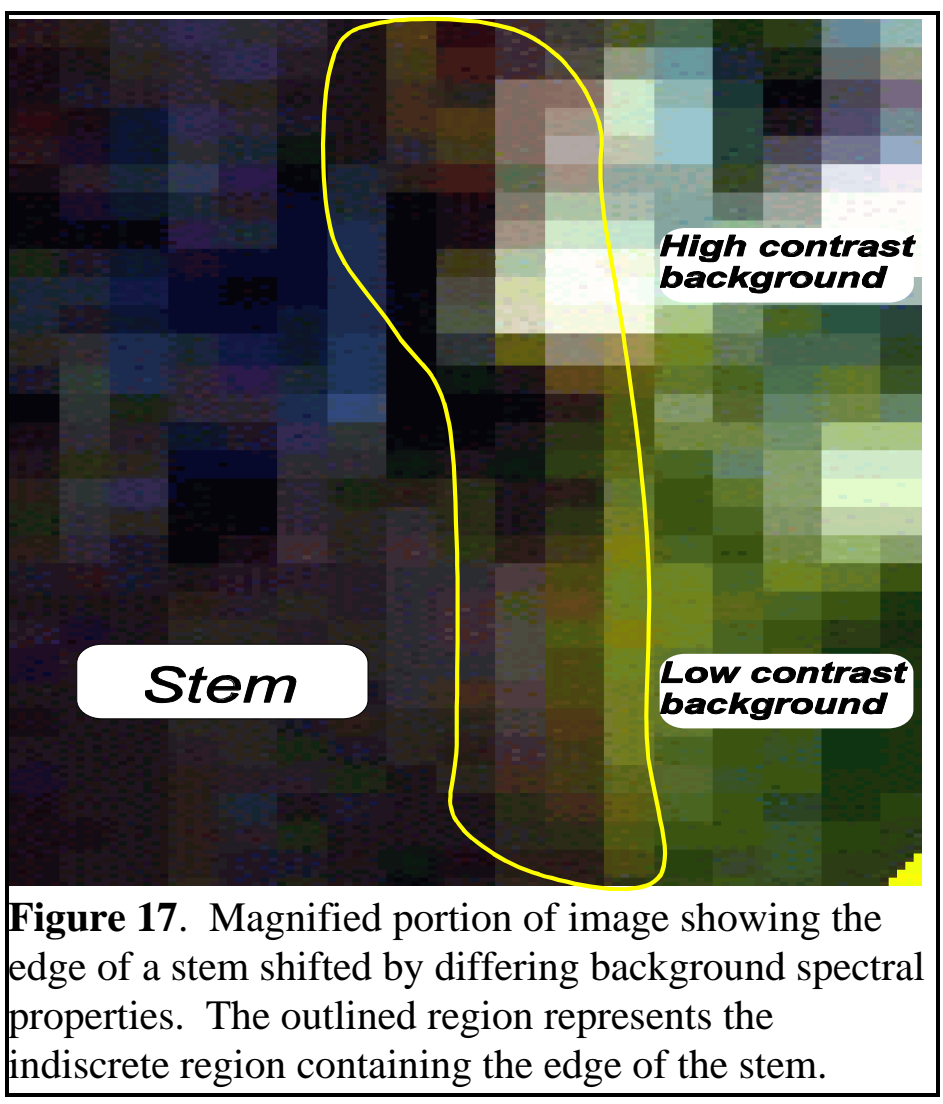

tape from being pulled taut for an accurate reading. Movement of the tape end at $B_{o}$ can cause error. The tape end was secured to the stem using a nail for this study to avoid this problem. Due to the pivot point of the tripod, the orientation of the focal point to the stem changes slightly as inclination angle is adjusted.

\section{Incorrect inclination angle}

measurement could be caused by misreading or inconsistent positioning 
of the clinometer relative to the camera body, or a mistake during recording or transferring data. The clinometer used in this study reported the angle with decreasing precision in percent up to $150 \%$ and graduations were present at a $\mathrm{x} / 66 \mathrm{ft}$ scale up to $200 \mathrm{ft}$. Data were recorded in the field to indicate which scale was being used. All angular data were later converted to radians using a spreadsheet before input to the diameter extraction program. Thus, incorrect recording in the field or entering into the computer are possible sources of error.

Image capture errors are primarily related to incorrect exposure or focus and were previously discussed in detail. Subjective (e.g. observer) error can occur at the image measurement stage. Attention was paid to locate the points used for diameter as near as possible to the pixel where there was the greatest amount of contrast from tree stem to background. In this experimental situation, where diameters at pre-defined heights had to be obtained, there were sometimes difficulties in locating edges due to obstructions or contrast situations where the edges were questionable (Figure 17). Even when there appears to be a clearly defined edge, the question remains whether to select the background pixel or the stem pixel coordinates. To offset any overlap problem an attempt was made to select towards the stem on one edge and away from the stem on the other. Often a larger "window" (increased area coverage) had to be used to incorporate contextual information to help infer point placement.

Finally, error can be caused by improper calculation. There may be more efficient or better ways to perform the diameter calculations. Inputs such as the row value for the base point $B_{o}$ could be changed to an image coordinate value to correct for angular error from the point to the lens center and to the stem axis rather than the parallel tangent. Alternate methods for diameter calculation could be applied as well as recognizing that a stem cross-section can only be referred to as a closed convex region rather than a circle or an ellipse (Brickell 1970). There is 
also the issue of rounding error, especially given the minute angle measurements. Double precision (15 significant digits) data types were used for the storage and calculations of floating point values. Given the other possible sources of error this should be minimal.

\section{Chapter 5}

\section{CONCLUSION}

\subsection{Collection Protocol}

One of the objectives of this project was to set forth data collection protocols. The method by which the data are collected may be optimized for differing informational needs. The methods tested in this paper were for total stem data capture and the only variable examined to establish a protocol was the distance between the camera station and the stem. In order to optimize the collection protocol certain data requirements must be decided. What portions of the bole need coverage? How many observations are needed per stem? Is spectral information required? Is there any possibility that these data will be used in the future or for another project? The answers to these questions are helpful for determining the number of images per stem, orientation to the stem, location on the stem, and camera settings.

\subsubsection{Image Scale}

Common sense must drive the decision of collection protocol. If there is some degree of probability that data collected today may serve the purposes of a future or separate project, then perhaps data should be collected with increased coverage, accepting a loss of precision and, possibly, time. For instance, in the past there have been changes in merchantability standards driven by technology developments which have allowed for increased utilization of the resource. Considering that this may happen again in the future, rather than capturing data at a large scale 
that may only cover 1 meter of the bole and increase precision by $5 \mathrm{~mm}$, the observer may opt to capture 10 meters of the bole (with a decreased precision), and allow for future comparisons or reduce future data collection costs.

With the camera model used for this study there are only two focal lengths that are "known"- $7 \mathrm{~mm}$ and $21 \mathrm{~mm}$. This only offers two options unless alternate methods are used for scale determination. The $21 \mathrm{~mm}$ focal length was not studied at the time of this publication. Unless there are complications with the interior camera parameters, this magnification should simply increase the possible measurement precision by a factor of 3 . This magnification could be used in conjunction with alternate means of distance determination to collect more precise diameters on certain portions on the stem - for instance, diameters at random heights for importance sampling (Gregoire et al. 1987, Van Deusen and Lynch 1987).

\subsubsection{Spectral Issues}

The need for obtaining spectral information may also drive collection protocol. Here incident energy (light) is of major importance. Time of day, time of year, weather conditions, orientation, and camera settings are concerns for spectral sensitivity. Twilight or overcast conditions as well as canopy conditions limit the incident energy. This is not necessarily a disadvantage as the shutter speed can be slowed to compensate and to capture more consistent reflectance than is often attainable under direct lighting conditions. There is also the option of using the camera flash, though the effective range reported in the camera manual is only 5 meters.

Backlit conditions are unfavorable for collecting spectral information (Figure 18). For collecting data with a backlit condition, manual exposure must be utilized to select a shutter speed of sufficient duration to capture the required signal strength. Auto-exposure will not allow 
for sufficiently long shutter speeds. If an object can be used to shield the direct radiation there is a better chance of capturing the spectral information that is desired (Figure 18). Direct radiation usually drowns out any reflective radiation that is desired and there is no recourse for this problem.

Direct lighting can provide the most detail for spectral discrimination. Problems can still occur if the exposure time is too long. Then the CCD can be saturated to an intolerable extent. Side lighting, causing uneven reflectance on the convex stem, can be troublesome if using any automated digital process requiring normalized reflectance.

\subsubsection{Seasonal Consideration}

The images collected for this study were collected in "pure" deciduous stands in the leafoff condition. This provided for a maximum level of contrast and visibility. It follows that seasonal considerations should also be made when determining the mode of collection. A previous study (Clark et al. 1998), using leaf-on images, done with the same camera and a slightly different method of diameter calculation resulted in a slight positive bias rather than the negative bias found in this study. The discrepancy between these results may partially be due to a higher frequency of low contrast situations caused by background vegetation.

\subsection{Comparison to Results of Other Studies}

Table 1 shows a sample of results from previous dendrometer tests. These results were reported in many different formats from units to percent and at different confidence levels from the standard deviation to $95 \%$ confidence tests for the normal and chi-square distributions. These tests were performed with different sample sizes from 26 to 300 diameter estimates taken from 1 to 87 stems at heights from 0 to 27.5 meters. Softwood species were the most prevalent 


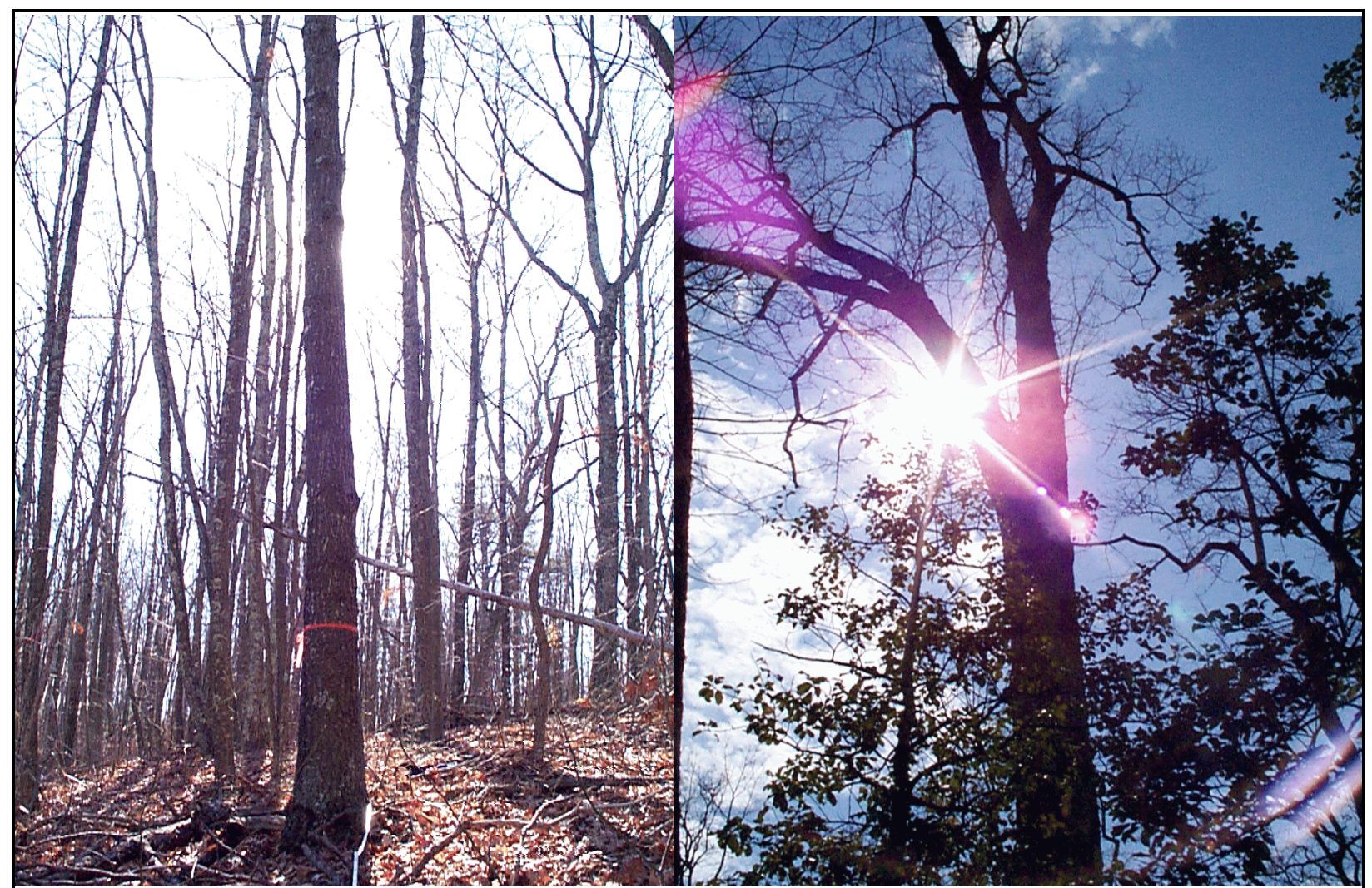

Figure 18. Two examples of a backlit condition. The left image demonstrates the technique of blocking the direct radiation and using an appropriate shutter speed to capture reflectance from the stem. This image can later be enhanced to more clearly distinguish the captured reflectance values. The right image is exposed to direct radiation which inundates the reflective radiation disallowing any useful spectral data collection.

among these tests and most of them marked the heights on the stem by some means. These

procedural differences warrant caution when making comparisons to other models that do not

account for the height determination error. The deliquescent branching structure in the hardwood stems observed in this study can have a severe impact on diameter over a short height interval (Figure 19).

The maximum anticipated error result for this camera (using the methods set forth in this paper) for heights from 1 to 5 meters is $25 \mathrm{~mm}$ (Table 4). This error is twice as large as Wheeler's (1962) pentaprism results and a little over three times that obtained by Bell \& Groman (1971) using the Barr and Stroud FP-12. 


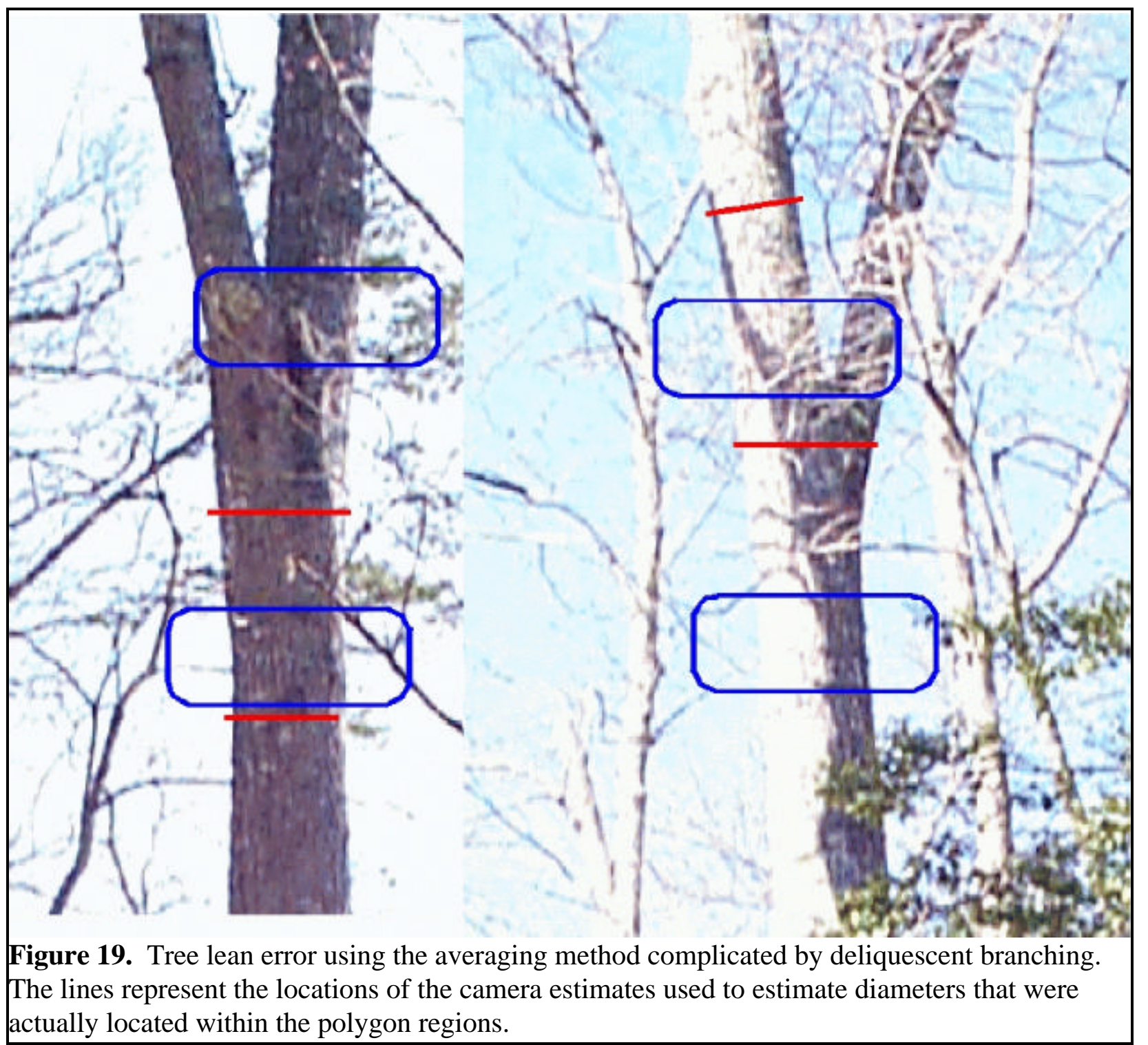

The range of deviations for heights up to $10 \mathrm{~m}$ is -86 to $106 \mathrm{~mm}$ which is considerably wider than the -17.8 to $33.0 \mathrm{~mm}$ pentaprism range on 120 conifer diameter observations acquired by Robbins and Young (1968) or even the $\pm 63.5 \mathrm{~mm}$ range obtained by Marsh (1952).

Standard deviation was the final statistic reported for many of the studies. Brickell (1976), Jeffers (1955), Bradshaw (1972), and Takahashi (1997) report standard deviations of 6.4, 15, 9.9, and $4.6 \mathrm{~mm}$, respectively. It appears that these deviations are correlated with the height range of the observations. The standard deviation of the $1.4 \mathrm{~m}$ results using the digital camera is 
$\pm 13.6 \mathrm{~mm}$. This is at least twice as large as findings using the dendrometers previously listed.

Garrett et al. (1997), using "percent inaccuracy”, calculated 95\% confidence intervals about the mean percent difference errors, with a normal distribution assumption. Assuming that this is calculated by multiplying the standard error by a $t$ value with the appropriate degrees of freedom for the sample collected, \pm 15.4 percent would be the result of all 1048 GMD estimates from this study. This is approximately double that of most instruments studied by Garrett et al. If only breast height percentage measurements are considered, the percent inaccuracy of the 90 observations (18 stems x 5 distances) captured in this study drops to \pm 7.9 percent which ranks well among the errors at breast height as reported by Garrett et al. Percent differences from different samples do not result in accurate comparisons as demonstrated by the volume case study (Section 4.5).

Grosenbaugh (1963) infers that two out of three volume estimates using the Barr \& Stroud FP9 dendrometer will be within 4.1 percent of estimates using a diameter tape. Under the normality assumption, using the 12 meter distance readings we can expect that two-thirds of the measurements will be within 7.7 percent. Again there is the problem of percentage comparisons, though it is not apparent from the data in Table 6 that stems with smaller volumes are correlated with larger percentage differences. This increased variation may be partially due to instrument precision or increased height range of samples in this study (merchantable heights of Grosenbaugh's 8 sample trees $15 \mathrm{~m}$ [1], $10 \mathrm{~m}$ [2], $5 \mathrm{~m}$ [5]).

\subsection{Areas For Further Development}

Operational implementation of this tool and procedure will require further research and development. Although data collection is rapid, data transfer and transformation in the post processing phase is currently problematic and time consuming. Ancillary data is recorded in the 
field by hand and then has to be manually transferred into a digital format. These steps are both time consuming and error prone. These problems could easily be overcome by interfacing the camera with digital clinometric and range finding devices which could be configured to output to each image's header file. With this associated information, software could be modified to automate much of the subsequent image registration processes.

Additionally, image reduction techniques for perpendicular camera stations should be developed to compensate for deviations of the stem axis from the datum (e.g., tree lean). This should provide more accurate stem height measurements and help adjust the camera-stem distance to improve the accuracy of the individual diameter estimates.

Camera calibrations should be performed in attempt to better describe lens distortions and interior orientation parameters. The non-metric nature of the camera reduces the reliability that these parameters remain constant. For this reason this calibration should be performed periodically to determine the extent and variability of the interior instabilities.

Efforts should be made to model edge displacement more effectively. Edge detection methods, such as those proposed by Jahne (1997), can be used to locate the image coordinates and subsequently the object space values of edges more precisely. Edge detection is vital to exploring automation of the stem measurement processes. If the computer can be trained to differentiate the appropriate stem, variation due to observer should be greatly reduced, and information extraction can be automated. To take advantage of the spatial information captured by the camera, the term "information" can be expanded beyond diameter and volume to include elements such as crown diameter, live crown ratio, form information (e.g. sweep/crook), as well as limited defect detection and characterization (Figure 20). 


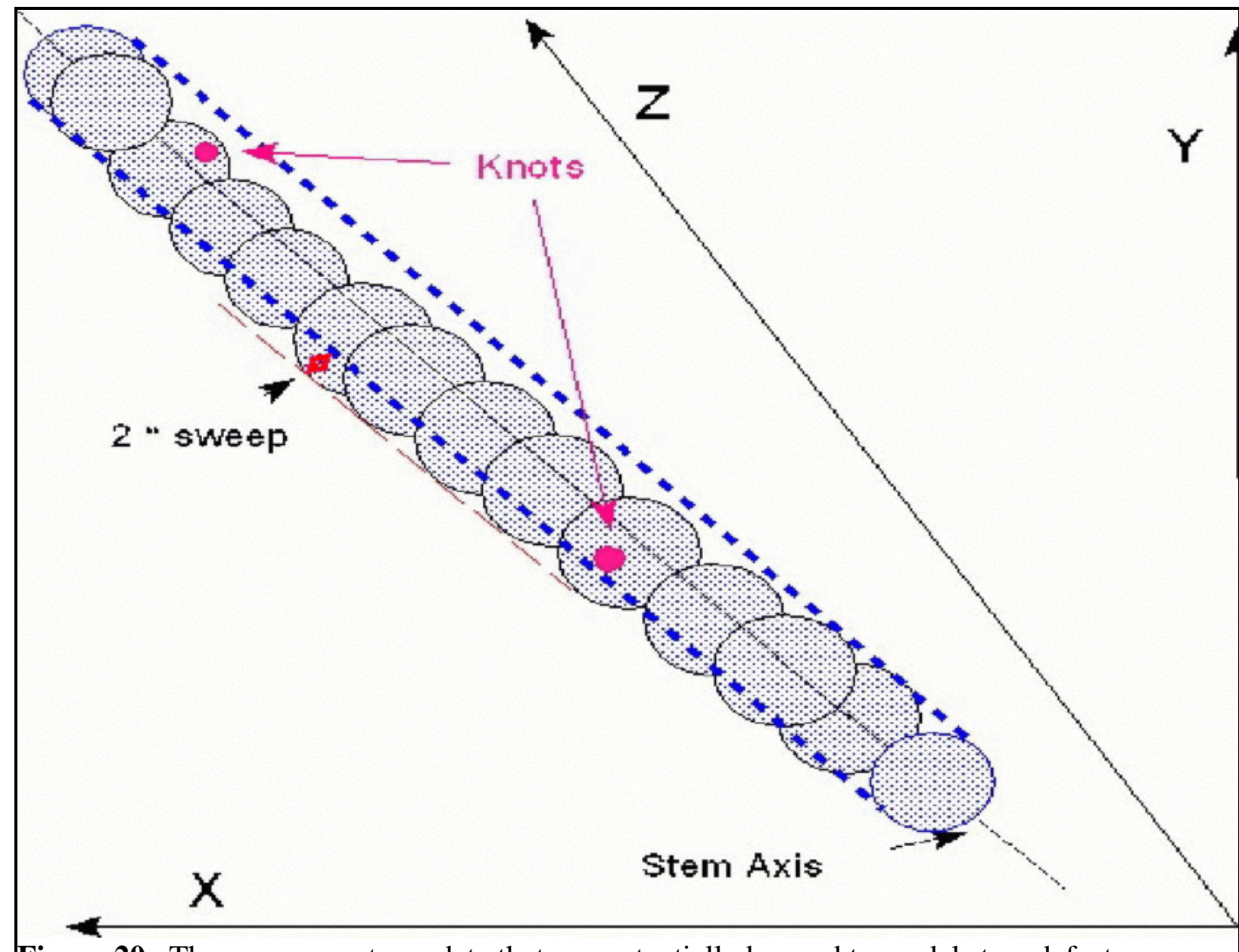

Figure 20. The camera captures data that can potentially be used to model stem defects.

A few modifications to the camera would be needed to improve its use for this purpose. A longer "panoramic" chip and associated lens, along with increased spatial resolution (which is rapidly occurring), would be helpful to optimize data capture in the direction of interest. As previously mentioned, angle- and range-finding components would be extremely helpful if integrated into a camera. A more durable (e.g. shock / weather resistant) casing would be needed for the camera to be serviceable for actual data collection on a regular basis. If these changes could be dealt with successfully, use of the camera in combination with hand-held field computers could provide 'on-the-fly' tree, stand, and even merchantability information. 


\subsection{Summary and Conclusion}

Detailed collection protocols need to be determined with consideration given to informational requirements. The conclusions of this study reveal that camera station distance should roughly be equal to the uppermost sample height to be sampled using the averaging method set forth in this study. Compensation for stem lean using perpendicular images may improve estimates obtained at nearer distances, though this is unexplored. It is doubtful, however, that error due to perspective at extreme inclination angles can be overcome. In general, shorter distances, when the inclination from the camera station to the measured point is less than 45 degrees, produce the most precise results. Longer distances provide more consistent estimates with a decrease in precision at lower stem heights.

The maximum anticipated error is $\pm 4 \mathrm{~cm}$ at the $95 \%$ confidence level. This value applies to geometric mean diameters for stem heights up to $20 \mathrm{~m}$ on upland red oak stems at a camera distance from the stem greater than $12 \mathrm{~m}$. This maximum anticipated error increases with height from $\pm 3 \mathrm{~cm}$ to $\pm 7 \mathrm{~cm}$ at stem heights from 1 to 20 meters. In this same height interval with a $12 \mathrm{~m}$ camera station distance the precision varies from 0.84 to $1.6 \mathrm{~cm}$.. The accuracy to instrument precision ratio is about 3 which is consistent with most other instruments assuming $2.5 \mathrm{~mm}$ increments.

Volume estimates will be within 8 percent of taped measurements of individual stems two times out of three, which is an improvement over the 20 to 28 percent possible using applicable volume equations. Often applicable volume equations are not easily obtained, especially if one considers the bias resulting from differing ranges and resolutions. The method used in this paper provides the ability to readily derive appropriate scaled volume equations.

More work needs to be done to control variation. Further experimentation using marked 
diameters and compensation for stem axis deviation from datum should remedy the gross errors greater than twice the image precision. Variation due to instrument precision can be reduced by pixel interpolation and increasing the focal length. The cost of this increased precision lies in collecting more images per stem to provide the same area coverage. Comparisons should be made between different species (e.g. softwood, bottomland hardwood) and stand conditions (e.g. densities, leaf-on) as well as differing lighting conditions (e.g. overcast, full sun). Software improvements should be implemented for greater automation and increased informational functionality. 


\section{LITERATURE CITED}

Adobe 1998. [WWW document]. URL

http://www.adobe.com/supportservice/custsupport/NOTES/2596.htm

Ashley, M.D., and R.E. Roger. 1969. Tree heights and upper stem diameters. Photogrammetric Engineering 35(6):136-146.

Avery, T.E., and H.E. Burkhart. 1983. Forest Measurements $\left(3^{\text {rd }}\right.$ ed.) McGraw-Hill, Inc., New York, 331 pages.

Bell, J.F., and W.A. Groman. 1971. A field test of the accuracy of the Barr and Stroud Type FP-12 optical dendrometer. The Forestry Chronicle 47(2):69-74.

Binot, J., D. Pothier, and J. Lebel. 1995. Comparison of relative accuracy and time requirement between the caliper, the diameter tape and an Electronic Tree Measuring Fork. The Forestry Chronicle 71(2):197-200.

Bitterlich, W. 1984. The Relascope Idea: Relative Measurements in Forestry. Commonwealth Agricultural Bureaux. Farnham Royal, England. 242 pp.

Bradshaw, F.J. 1972. Upper stem diameter measurements with the aid of $35 \mathrm{~mm}$ photographs. Australian Forest Research. 6(1):17-20.

Brickell, J.E. 1970. More on diameter tape and calipers. Journal of Forestry 68(3):169-170.

Brickell, J.E. 1976. Bias and Precision of the Barr and Stroud Dendrometer Under Field Conditions. USDA Forest Service Research Paper INT-186. Intermountain Forest and Range Experiment Station. Ogden, Utah. 46 pp.

Clark, A., III and J.G. Schroeder. 1986. Weight, Volume, and Physical Properties of Major Hardwood Species in the Southern Appalachian Mountains. USDA Forest Service Research Paper SE-253. Southeast Forest Experiment Station. Asheville, North Carolina. 63 pp.

Clark, N., R.H. Wynne, D.L. Schmoldt, P.A. Araman, and M. Winn. 1998. Use of a non-metric digital camera for tree stem evaluation. Technical Papers, $64^{\text {th }}$ Annual Meeting of the American Society for Photogrammetry and Remote Sensing. Tampa, Florida, 13 pp.

Clarke, T.A. 1995. An analysis of the prospects for digital close-range photogrammetry. ISPRS Journal of Photogrammetry and Remote Sensing 50(3):4-7.

Cost, N.D. 1979. Multiresource Inventories: A Technique for Measuring Volumes in Standing Trees. USDA Forest Service Research Paper SE-196. Southeast Forest Experiment Station. Asheville, North Carolina. 18 pp. 
Crosby, P., J.P. Barrett, and R. Bocko. 1983. Photo estimates of upper stem diameters. Journal of Forestry 81(12):795-797.

Evans, D., and P. Hardwick. 1997. Digital camera and enhancements. Technical Papers, $63^{\text {rd }}$ Annual Meeting of the American Society for Photogrammetry and Remote Sensing. Seattle, Washington, Vol. 4, pp. 486-492.

Fairweather, S.E. 1994. Field tests of the Criterion 400 for hardwood tree diameter measurements. The Compiler 12(1):27-29.

Forbes, R.D. 1955. Forestry Handbook. The Ronald Press Company. New York. 23 sections.

Freese, F. 1960. Testing accuracy. Forest Science 6:139-145.

Fryer, John G. 1989. Camera Calibration in Non-Topographic Photogrammetry. pp. 59-69. In Non-Topographic Photogrammetry, $2^{\text {nd }}$ Edition. H.M. Karara, editor, American Society for Photogrammetry and Remote Sensing. Falls Church, Virginia.

Garrett, S., J.J. Kempf, and R.L. Copstead. 1997. Instruments for Measuring Stem Diameters. Publication \# 9724 1801. San Dimas Technology and Development Center.

Gregoire, T.G., H.T. Valentine, and G.M. Furnival. 1987. Sampling methods for estimating stem volume and volume increment. Forest Ecology and Management 21:311-323.

Gregoire, T.G., M.R. Reynolds. 1988. Accuracy testing and estimation alternatives. Forest Science 34(2):302-320.

Grosenbaugh, L.R. 1963. (Optical Dendrometers For Out-Of-Reach Diameters: A Conspectus And Some New Theory.) Forest Science. Monograph \#4. Society of American Foresters. 47 pages.

Holland, A. 1997. Solid State Devices Group, part of the X-ray astronomy group at the Dept. of Physics and Astronomy, University of Leicester, UK.[WWW document]. URL http://www.star.le.ac.uk/solidstate/ccd.html

Jähne, Bernd. 1997. Practical Handbook on Image Processing for Scientific Applications. CRC Press LLC. Boca Raton, Florida. 589 pages.

Jeffers, J.N.R. 1955. Barr \& Stroud Dendrometer Type F.P.7. Great Britain Forestry Commission Report on Forest Research for the Year Ended March, 1955. pp. 127-136.

KODAK 1997. [WWW document]. URL http://www.kodak.com/global/en/service/faqs/faq1518.shtml\#q15

Marsh, E.K. 1952. The Measurement of Standing Sample Trees. Union of South Africa Department of Forestry, Pretoria. 8 pp. 
McClure, J.P. 1969. The Mirror Caliper, A New Optical Dendrometer. USDA Forest Service Research Note SE-112. Southeast Forest Experiment Station. Asheville, North Carolina.

Ott, R.L. 1993. An Introduction to Statistical Methods and Data Analysis( $4^{\text {th }}$ ed). Duxbury Press. Belmont, California. 1183 pages.

Parker, R.C. 1997. Nondestructive sampling applications of the Tele-Relaskop in forest inventory. Southern Journal of Applied Forestry 21(2):75-83.

Rennie, J.C., and J.D. Leake, Jr. Determining Girard form class in central hardwoods. Northern Journal of Applied Forestry 14(4):202-206.

Robbins, W.C., and H.E. Young. 1968. An evaluation of the McClure and Wheeler optical calipers. The Forestry Chronicle 44(4):16-18.

Takahashi, M., K. Saito, N. Shiraishi, T. Iehara, and F. Takahashi. 1997. A photo based measurement system using a measuring camera. Japan Society of Forest Planning Press 3:1-9.

TWAIN. 1998. [WWW document]. URL http://www.twain.org/about.htm

University of Texas Health Science Center at San Antonio. 1998. [WWW document]. URL http://ddsdx.uthscsa.edu/dig/itdesc.html

Van Deusen, P.C., and T.B. Lynch. 1987. Efficient unbiased tree-volume estimation. Forest Science 33:583-590.

Wheeler, P.R. 1962. Penta prism caliper for upper-stem diameter measurements. Journal of Forestry 60:877-878.

Williams, M.S., W.A. Bechtold, and V.J. LaBau 1994. Five instruments for measuring tree height: An evaluation. Southern Journal of Applied Forestry 18(2):76-82. 


\section{Appendix A - Measurement Derivation}

The inputs required to calculate a diameter at a certain height are:

From Field Data:

$\theta=$ camera inclination angle

$\mathrm{L}_{\mathrm{o}}=$ distance from center of lens to point $\mathrm{B}_{\mathrm{o}}$ in inches to the nearest 0.5 "

From ImagelUser input:

$B_{i}=$ row value of point $B_{o}$ tree height at point $B_{o}$ $s_{\text {image }}=$ image distance representing the diameter at stem height $i$

$h_{o}=$ stem height of desired measurement

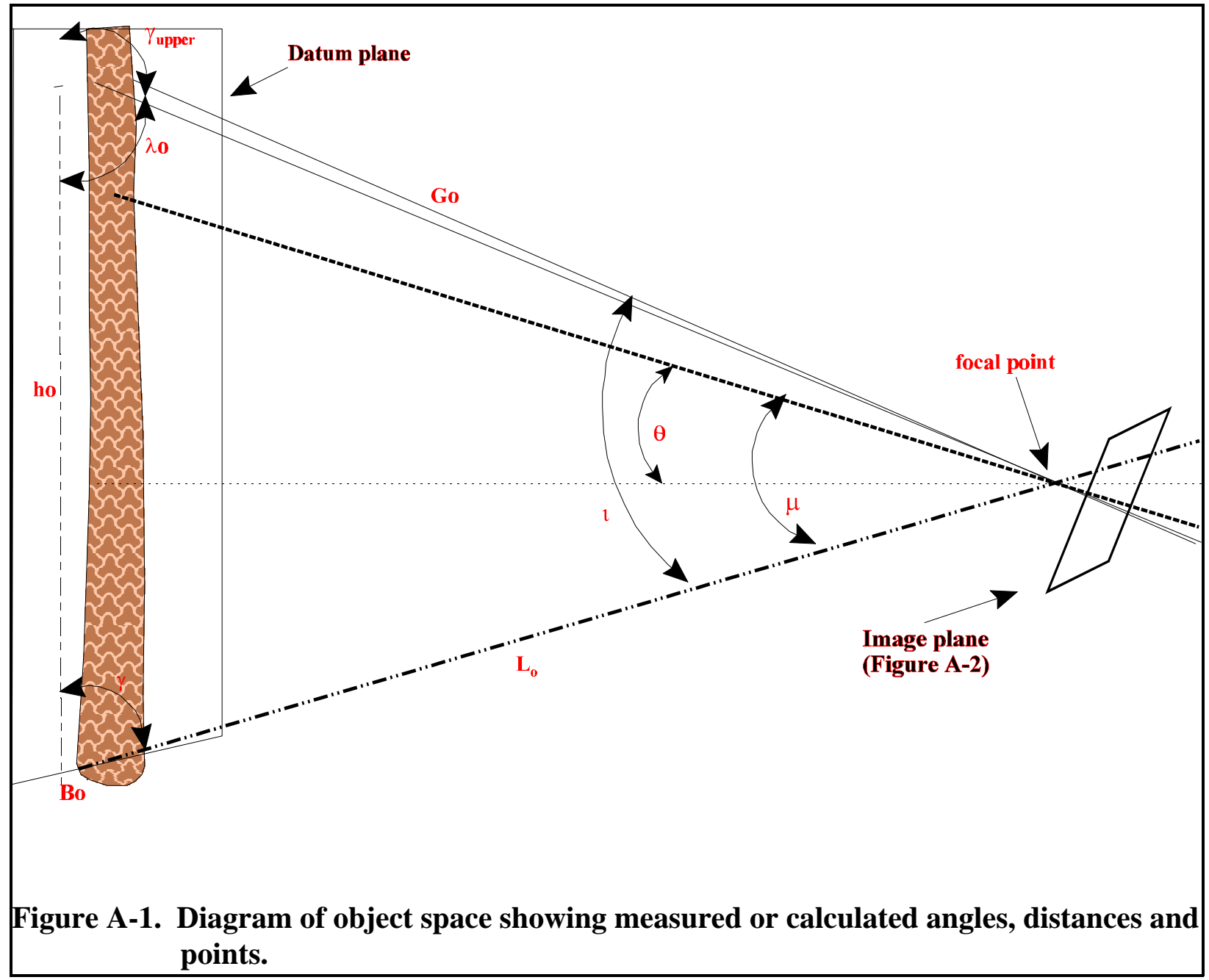


Photographic measurement derives its proof from the law of similar triangles. The magnitude and direction of the angle between two points in object space with vertex at the focal point is equal to the angle between those same points on the image with vertex at the focal point. Since oblique imagery is used, the legs of the triangle formed by these points in object space are not congruent to these legs in image space. The datum planes (of the two perpendicular images) are assumed to intersect along a plumb line through a point corresponding to the stem axis at tree height $\left(B_{o}\right)$. Lens and CCD aberrations are assumed to be negligible given other factors and are not accounted for in these calculations.

Directional diameter estimates are calculated by doubling the radius calculated from a single image (Equation A-1).

$$
\text { Diameter }_{\text {directional }}=2 r
$$

The radius is the sine of the angle $(\phi)$ (Figure A-1) formed by the stem axis, the focal point, and the edge of the stem in the plane containing the focal point and the two edge points perceived to represent the diameter, multiplied by the object space distance between the focal point and the stem $\operatorname{axis}\left(G_{o}\right)($ Equation A-5).

$$
r=\sin \frac{\phi}{2} \times G_{o}
$$




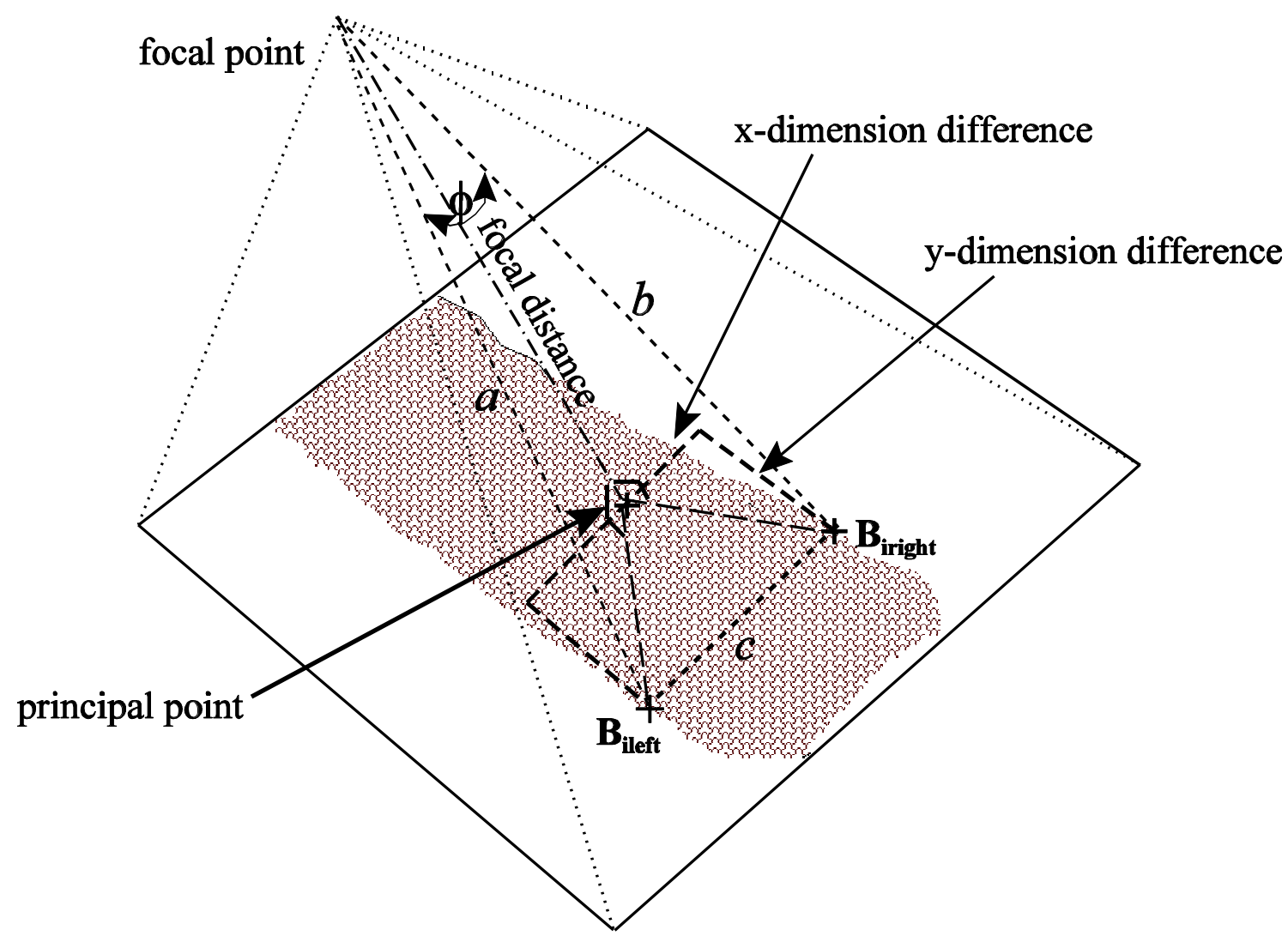

Figure A-2. Image space showing relationship of angle $\phi$ and image points $\mathrm{B}_{\text {ileft }}$ and $\mathrm{B}_{\text {iright }}$.

By Law of sines:

$$
\phi=\arcsin \left[\frac{2 \sqrt{s(s-a)(s-b)(s-c)}}{a b}\right]
$$

where $a, b, c=$ lengths of segments between the focal point and the image points $\mathrm{B}_{\text {ileft }}$ and $\mathrm{B}_{\text {iright}}$, these are all calculated using the Euclidean distance formula (Equation A-4)

$$
s=1 / 2(a+b+c)
$$

$$
h=\sqrt{i^{2}+j^{2}}
$$

substituting the focal length for $i$ and the distance from the principle point for $j$ 
\{again using the Euclidean distance formula, with $i$ as the difference between the $\mathrm{x}$-dimensions and $j$ the difference between the $\mathrm{y}$ dimensions of the principle point and the image point $(i)$.$\} , to find the image distance between the focal point and$ the image points. And substituting the difference in the image x-dimensions for $i$ and difference in the image $y$-dimensions for $j$ in order to calculate the distance between the left and right points on the image.

$$
G_{o}=\sqrt{L_{o}^{2}+h_{o}^{2}-2 L_{o} h_{o} \cos \gamma}
$$

where $h_{o}=$ stem distance between defined height and $B_{o}$

$\gamma=$ angle between datum plane and ray $L_{o}$ (Equation A-6)

If $B_{o}$ is below the principle point on the image then

$$
\begin{aligned}
& \theta<0 \rightarrow \gamma=90^{\circ}-(\mu-\theta) \\
& \theta>0 \wedge \mu<\theta \rightarrow \gamma=180^{\circ}-\left(90^{\circ}-(\theta-\mu)\right) \\
& \left.\theta>0 \wedge \mu>\theta \rightarrow \gamma=180^{\circ}-\left(90^{\circ}-\theta\right)+\mu\right)
\end{aligned}
$$

If $B_{o}$ is above the principle point on the image then

$$
\begin{aligned}
& \theta>0 \rightarrow \gamma=180^{\circ}-\left(\left(90^{\circ}-\theta\right)+\mu\right) \\
& \theta<0 \wedge \mu>\theta \rightarrow \gamma=180^{\circ}-\left(90^{\circ}-(\theta+\mu)\right) \\
& \theta<0 \wedge \mu<\theta \rightarrow \gamma=\left(90^{\circ}-(\mu-\theta)\right)
\end{aligned}
$$

where $\mu=$ angle between rays $L_{i}$ and $f$ which is also equivalent to the angle between the camera axis and $B_{o}$ (Equation A-7)

$$
\mu=\arctan \left(\frac{m_{i}}{f}\right)
$$

where $f=$ focal length (at maximum wide angle $7.0 \mathrm{~mm}$ )

$m_{i}=$ image distance from principle point to point $B_{i}$

$$
\lambda_{o}=180^{\circ}-(\gamma+1)
$$

Calculation of Image Row Values 
The row values $\left(y_{i}\right)$ for each $h_{o}$ are calculated by Equation A-9

$$
y_{i}=B_{i}-\left(\frac{h_{i}}{p}\right)
$$

where $h_{i}=$ image measurement in inches between image point $B_{i}$ and a desired point

(Equation A-10)

$p=$ the image pixel dimension $(.005 \mathrm{~mm})$

$$
h_{i}=\frac{\sin 1 \times L_{i}}{\sin \lambda_{i}}(\mathrm{~A}-10)
$$

where $l=$ angle between rays $L_{i}$ and $G_{i}$ or between $L_{o}$ and $G_{o}$ (Equation A-11)

$L_{i}=$ distance from the focal point to the point $B_{i}$ on the image plane (Equation A-12)

$\lambda_{i}=$ angle between the image plane and ray to stem axis at height $i$ (Equation A-13)

$$
\begin{gathered}
\imath=\arcsin \left(\frac{\sin \gamma \times h_{o}}{G_{o}}\right) \\
L_{i}=\frac{f}{\cos \mu} \\
\lambda_{i}=180^{\circ}-(\Gamma+\imath)
\end{gathered}
$$

where $\Gamma=$ angle between the image plane and ray to point $\left(B_{i}\right)$ (Equation A-14)

$$
\Gamma=90^{\circ}-\mu
$$

Calculation of $G_{o a}$ and $\gamma_{\text {upper }}$ For a Given Row Value

$G_{o a}$ (Equation A-15) and $\gamma_{\text {upper }}$ (Equation A-20) must be calculated for images not containing the point $B_{o}$.

$$
G_{o a}=\frac{L_{o} \times \sin \gamma_{\text {lower }}}{\sin \lambda_{o a}}
$$

where $\lambda_{o a}=$ angle between datum plane and ray intersecting point $a$ in the lower image (Equation A-16)

$L_{o}=$ distance from center of lens to point $\mathrm{B}_{\mathrm{o}}$ in inches to the nearest 0.5 " for the lower image

$\gamma_{\text {lower }}=$ angle between datum plane and ray $L_{o}$ for the lower image (Equation A-6)

$G_{o a}$ then serves as $L_{o}$ for the upper image 


$$
\lambda_{\text {oa }}=180^{\circ}-\left(\mathrm{l}_{a}+\gamma_{\text {lower }}\right)
$$

where $\mathbf{l}_{a}=$ angle between ray intersecting $B_{o}$ and ray intersecting $a$ (Equation A-17)

$$
\iota_{a}=\frac{\arcsin \left(\sin \Gamma \times h_{i a}\right)}{G_{i a}}
$$

where $h_{i a}=$ image measurement in inches between $B_{i}$ and $a_{\text {image }}$ (Equation A-18)

$G_{i a}=$ image space distance between focal point and $a_{\text {image }}($ Equation A-19)

$$
h_{i a}=\left(y_{i a}-y_{B_{i}}\right) \times p
$$

where $y_{i a}=$ row value corresponding to $a_{\text {image }}$

$y_{B i}=$ row value corresponding to $B_{i}$

$p=$ the image pixel dimension $(.005 \mathrm{~mm})$

$$
\begin{gathered}
G_{i a}=\sqrt{{h_{i a}{ }^{2}+L_{i}^{2}-2 h_{i a} L_{i} \cos \Gamma}^{2}} \\
\gamma_{\text {upper }}=180-\lambda_{\text {oa }}
\end{gathered}
$$

$\gamma_{u p p e r}$ serves as $\gamma$ in the calculations involving the upper image 


\title{
Appendix B - Diameter Extraction Source Code (C++)
}

\author{
//1010101010101010101010101010101010101010101010101010 \\ //10 Neil Clark $\quad 10$ \\ //1010101010101010101010101010101010101010101010101010 \\ // \\ //PROGRAM NAME: Diameter Extraction Software \\ //
}

//SYSTEM: Borland C++ v. 5.0 run on Intel Pentium $(166 \mathrm{mH})$ under Windows 95

//

//DATE: 8/20/98

$/ /$

//DESCRIPTION:

//

//This menu driven program uses an image data file and a stem/height file as

//inputs. The image data file must have the following structure: image

//name[int], tree number [int], side number [int], distance [float], nominal

//distance [int], inclination angle in radians [float], row value of point $\mathrm{Bi}$

//in image [int], object space height of point Bi [int[, uppermost object space

//height [f], lowest object space height [float], gamma [float], capgamma

//[float], and L_i [float]. The last five values are computed by the program,

//but values must be present in the input file in order that the file should be

//properly read in regardless of the order of menu choices. The first record

//should be a set of dummy values.

// The stem/height file must have the following structure: tree number [int]

//and height [float].

// call libraries to be used

\#include < fstream.h>
\#include <iomanip.h>
\#include < math.h>
\#include <string.h>
\#include < stdlib.h>
\#include < assert.h>

// used for file $\mathrm{I} / \mathrm{O}$

// used for output formatting

$/ /$ used for trig functions

// used for string manipulations

// declare global variables and define structs

const MAXHEIGHTS $=1000$;

const MOSTIMAGES = 1500;

const double $\mathrm{PI}=3.1415927$;

double $\mathrm{f}=7000.0$, pixdim $=5.0$;

double height[MAXHEIGHTS];

char response;

char file[80];

char basefile[80];

char diamfile[80];

typedef struct

\{ int imagenum;

int treenum;
// maximum number of stem/height records allowed

// maximum number of image records allowed

// constants for focal length and pixel

// dimension in microns

// array of height records

// one character user input variable

// string array for filename

// string array for filename

// string array for filename

//definition of data structure for each student

// identification number for each image

// identification number for each stem 


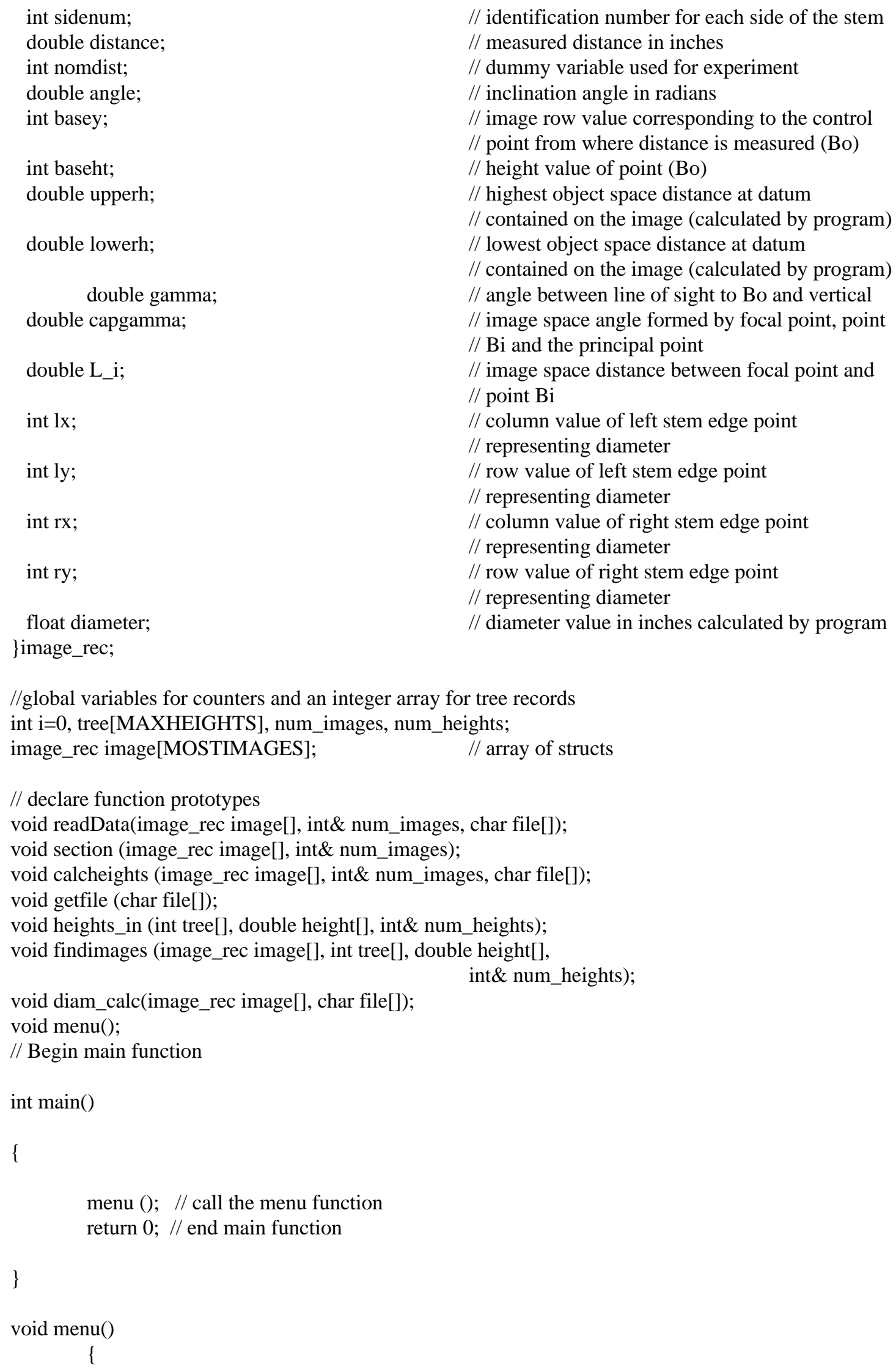


char choice; $\quad$ // local variable for single character input

// menu options output to screen

cout $<<" \quad$ Tree Measurement System" $<<$ endl

$<$ " $\wedge \wedge \wedge \wedge \wedge \wedge \wedge \wedge \wedge \wedge \wedge \wedge \wedge \wedge \wedge \wedge \wedge \wedge \wedge \wedge \wedge \wedge \wedge \wedge \wedge \wedge \wedge \wedge \wedge \wedge \wedge \wedge \wedge \wedge \wedge \wedge \wedge \wedge \wedge \wedge \wedge \wedge \wedge \wedge \wedge \wedge \wedge \wedge \wedge \wedge \wedge \wedge \wedge \wedge \wedge \wedge \wedge \wedge \wedge \wedge \wedge \wedge \wedge \wedge "$

$<$ endl

$<<\quad|||||||||||||||||||||||||||||||||||||||||||||||||||||||| \mid "$

$<<$ endl

$<<" \quad$ MENU "

$<$ endl

$<<$ endl

$<<" 1)$ Select image input file"

$<<$ endl

$<<" 2$ ) Select tree/height file"

$<$ endl

$<<" 3)$ Register images"

$<<$ endl

$<<" 4)$ Find images and output row values"

$<<$ endl

$<<$ " 5) Calculate diameters"

$<<$ endl

$<<$ " 6) Quit"

$<<$ endl

$<<$ endl;

// read input character

cin $>$ choice;

// evaluate input choice

switch (choice) \{

// if the user enters the character '1' call readData function to

// read in the image data file then return to the menu

case '1' : readData (image, num_images, basefile);

$/ /$ call function to read data from file

menu ();

//and store in struct

break;

// if the user enters the character ' 2 ' intialize tree and height

// arrays and call heights_in function to read in the stem/height

// file. Then call menu function.

case '2' : for ( $\mathrm{i}=0$; $\mathrm{i}<\mathrm{MAXHEIGHTS;} \mathrm{++i)}$

$\{$ tree $[\mathrm{i}]=0$;

height $[i]=0$;

\}

heights_in (tree,height,num_heights);

menu ();

break;

// if the user enters the character ' 3 ' call section function

// which calculates some image orientation variables, control

// moves on to the calcheights function which can be used to 
// modify certain variables interactively, control returns to

// the menu function.

case ' 3 ' : section (image, num_images);

calcheights (image,num_images, file);

menu ();

break;

// if the user enters the character ' 4 ' the findimages function

// is called which searches the image records for the trees and

// heights that were read into their appropriate arrays by the

// heights_in function. An output file is produced which lists

// the image names and row values that correspond to the tree and

// height values.

case '4' : findimages (image,tree,height,num_heights);

menu ();

break;

// if the user enters the character ' 5 ' the diam_calc function is

// called which reads the output file created by the findimages

// function and calculates the diameters for each record and

// outputs these diameters to another file.

case '5' : diam_calc(image, diamfile);

menu ();

break;

// if the user enters the character ' 6 ' control is returned to

$/ /$ the main function and the program ends.

case '6' : return;

// if the user input does not match any of the above conditions

// control cycles back to the menu function until an acceptable

// value is entered.

default : menu ();

\}

\}

// This function prompts the user to enter the filename of the image input data // file. Upon successful filename entry the function reads data into the // image struct. It also counts the number of imagess and passes the value by // reference to the num_images memory location.

$/ /$

// This function takes a image_rec struct, a integer variable, and a character

// array as parameters.

void readData(image_rec image[], int\& num_images, char file[])

\{

// declare and initialize local variables

// Index = integer variable at serves as a counter for image records.

// tnum, snum, by, bh, and nomdist are temporary integer variables 
// that hold the input values for tree number, side number, the row // value of the base point, the object space height of the base point, // and the dummy variable of the nominal distance, respectively.

// dist, ang, upperh, lowerh, gamma, capgamma, and L_i are temporary // double precision variables for the transfer of distance, angle, // highest object space height, lowest object space height, object // space angle formed by vertical plane, basepoint, and line of sight // to basepoint, image space angle formed by focal point, basepoint on // image and principal point, and the image space distance between $/ /$ the focal point and the basepoint on the image.

// imgname is a temporary integer variable for the transfer of the // image identification number from the input file to the struct.

// fieldin is a local string for holding the filename input

int Index $=0$, tnum $=0$, snum $=0$, by $=0$, bh $=0$, nomdist; double dist $=0.0$, ang $=0.0$, upperh $=0.0$, lowerh $=0.0$, gamma $=0.0$, capgamma $=0.0$, int imgname $=0$;

char fieldin[80];

ifstream imgfile;

// prompt user to input the image filename cout << "Enter image file name: "; getfile(fieldin); // run filename function imgfile.open(fieldin); // open the input file

//PRIME READ to read first record

$$
\begin{aligned}
& \text { imgfile }>\text { imgname }>>\text { tnum }>\text { snum }>\text { nomdist }>\text { dist }>\text { ang }>\text { by }>\text { bh } \\
& \text { >> upperh } \gg \text { l lowerh }>>\text { gamma } \gg \text { capgamma > L_i; }
\end{aligned}
$$

// read until the end of the file is encountered while (imgfile)

\{

$$
\text { imgfile.ignore (100, 'In'); // advance to next record }
$$

image[Index].imagenum = imgname; // assign read variables to

appropriate struct locations

image [Index].treenum = tnum;

image[Index].sidenum = snum;

image [Index].distance $=$ dist;

image[Index].nomdist $=$ nomdist;

image[Index $]$.angle $=$ ang;

image[Index].basey = by;

image[Index].baseht $=$ bh;

image[Index].upperh = upperh;

image[Index].lowerh = lowerh;

image[Index].gamma = gamma; 


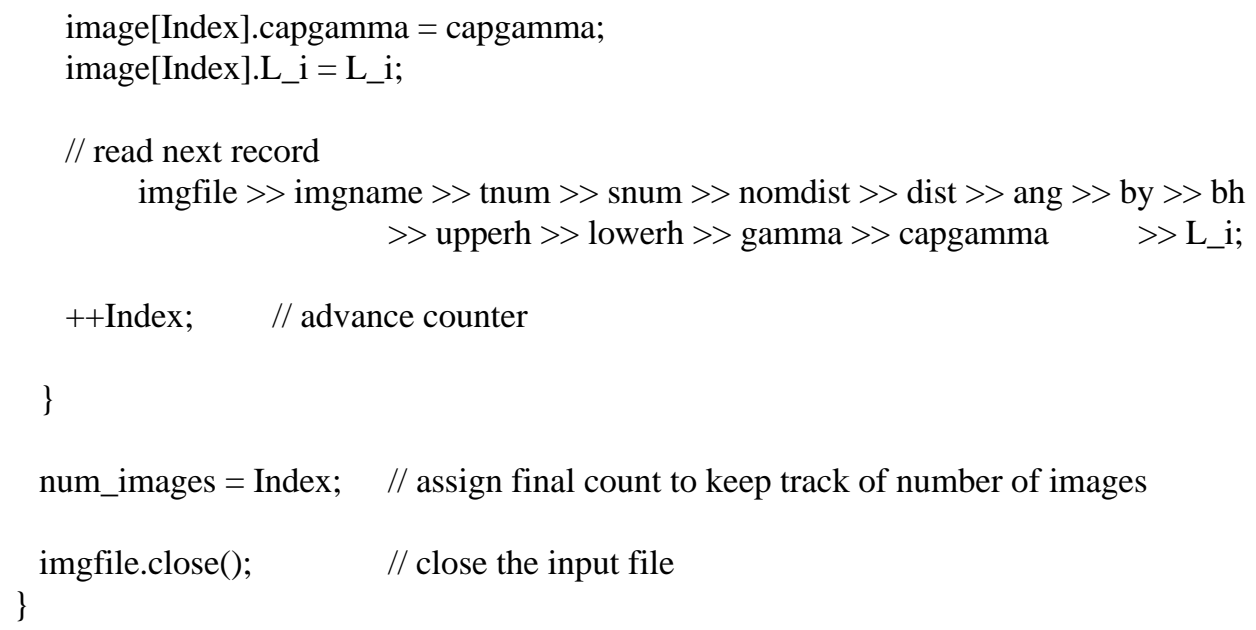

// This function goes through all of the image records and recalculates and // assigns values to the upperh, lowerh, gamma, capgamma, and L_i.

void section (image_rec image[], int\& num_images)

\{

// declare and initialize local variables

// $\mathrm{ANG}=$ angle between principal point and point lying in the principal

// $\quad$ plane at the vertical edge (rows 0 and 1280) of image

// iota_upper $=$ angle formed by the focal point, a point having the row

// value corresponding to the basepoint $(\mathrm{Bi})$ on the image and lying on the // principal axis, and a point on the nearest vertical edge also lying on // the principal axis.

// iota_lower $=2 \times$ ANG - iota_upper

// gamma = // angle between line of sight to Bo and vertical

$/ / \mathrm{mu}=$ angle formed by principal point, focal point and point lying on

// the principal axis with row value corresponding to point $\mathrm{Bi}$

// lambda_o_upper = object space angle formed by the datum plane and the $/ /$ ray imaged on the lowest row $(0)$

// lambda_o_lower = object space angle formed by the datum plane and the $/ /$ ray imaged on the highest row (1280)

// h_o = object space length between point Bo and highest and lowest

// points on datum that can be imaged by the camera

// cgamma = dummy variable

const double $\mathrm{ANG}=\mathrm{PI} / 2.0$-atan(7.0/3.2);

double iota_upper, iota_lower, gamma, mu, lambda_o_upper, lambda_o_lower; double h_o, cgamma;

// calculate for all image records for $(\mathrm{i}=0 ; \mathrm{i}<$ num_images; $++\mathrm{i})$

// calculations when $\mathrm{Bi}$ lies above the principal point in image if (image[i].basey < 641)

\{

// mu equals the arctangent of the image space distance between the

// principal point divided by the focal length

$\mathrm{mu}=\operatorname{atan}((640$-image[i].basey $) * \operatorname{pixdim} / \mathrm{f})$;

// gamma calculations dependent on location of Bo in relation to

// horizontal plane containing focal point,gamma greater than PI/2 
// if Bo above focal point height

// if inclination is positive, then gamma is the supplementary

// angle of the sum of mu and the inclination angle of the camera

if (image[i].angle $>0$ )

gamma = PI - (PI/2.0 - (image[i] $\cdot$ angle $+\mathrm{mu})$ );

// if inclination is negative and the mu is greater than the

// absolute value of the inclination angle, then gamma is

// calculated in the same way as above

else if $(\mathrm{mu}>(-1 *$ image[i].angle $))$

gamma = PI - (PI/2.0 - (mu + image[i].angle $)$;

// if inclination is negative of greater magnitude than mu, then

// gamma is the complement of the difference of those two angles

else gamma $=\mathrm{PI} / 2.0-(\mathrm{mu}-$ image[i]. angle $)$;

// the angle between the base point and the highest imaged point

// along the principal axis is calculated by the difference between

// ANG (which is angle between the principal point and the image

// edge lying in the principal plane) and mu (the angle between the

$/ /$ principal point and the image point $\mathrm{Bi}$ also lying in the

// principal plane.

iota_upper $=\mathrm{ANG}-\mathrm{mu}$;

// lambda_o_upper is computed as 180 degrees minus the sum of gamma

// and iota_upper

lambda_o_upper = PI - iota_upper - gamma;

// for cases where the sum of iota_upper and gamma are greater than

// 180 degrees a sufficiently small value is assigned for

// lambda_o_upper

if (lambda_o_upper < 0 )

lambda_o_upper $=0.0001$;

// to calculate the stem distance from the base point to the upper

// edge of the stem, the law of sines is used with the image

// distance as a leg and iota_upper and lambda_o_upper as angles

h_o = (sin(iota_upper) $*$ image[i].distance)/sin(lambda_o_upper);

// add this stem length to the base height for the actual object

// space height at datum

image[i].upperh $=$ h_o + image[i].baseht;

// the angle between the base point and the lowest imaged point

// along the principal axis is calculated by the sum of

// ANG (which is angle between the principal point and the image

// edge lying in the principal plane) and mu (the angle between the

// principal point and the image point $\mathrm{Bi}$ also lying in the

// principal plane.

iota_lower = mu + ANG;

// lambda_o_lower is computed as 180 degrees minus the sum of

// the supplement of gamma and iota_upper

lambda_o_lower =PI - ((PI - gamma) + iota_lower);

// to calculate the stem distance from the base point to the lower

// edge of the stem, the law of sines is used with the image 
// distance as a leg and iota_lower and lambda_o_lower as angles

$\mathrm{h} \_\mathrm{o}=(\sin ($ iota_lower $) *$ image $[\mathrm{i}]$.distance $) / \sin ($ lambda_o_lower $)$;

// subtract this stem length to the base height for the actual object

// space height at datum

image[i].lowerh = image[i]. baseht $-\mathrm{h} \_\mathrm{o}$;

// assign new gamma to struct

image[i].gamma = gamma;

// 180 degrees minus the complement of mu is assigned to the

// capgamma of the image

image[i].capgamma $=$ PI $-(\mathrm{PI} / 2.0-\mathrm{mu})$;

// the image space distance from the focal point to the basepoint is

// the quotient of the focal point over the cosine of mu.

\}

image[i].L_i $=\mathrm{f} / \cos (\mathrm{mu})$;

// calculations when Bi lies below the principal point in image

else

\{

$/ /$ mu equals the arctangent of the image space distance between the

// principal point divided by the focal length

$\mathrm{mu}=\operatorname{atan}(($ image$[\mathrm{i}] \cdot$ basey-640)*pixdim/f);

// if inclination is negative, then gamma is the complement of the

// difference of mu and the inclination angle of the camera

if (image[i].angle $<0$ )

gamma $=\mathrm{PI} / 2.0$ - (mu - image[i].angle $)$;

// if $\mathrm{mu}$ is less than the inclination angle, then gamma is 180

// degrees minus the complement of the difference between the

$/ /$ inclination angle and mu.

else if (mu < image[i].angle)

gamma $=$ PI $-(P I / 2.0$ - (image [i].angle $-\mathrm{mu})$ );

// if the inclination angle is positive and less than mu, then

// gamma is the complement of the difference between the two angles. else gamma = PI/2.0 - (mu - image[i].angle);

// iota upper becomes the sum of the two angles

iota_upper $=\mathrm{mu}+\mathrm{ANG}$;

// lambda_o_upper is 180 degrees less the sum of gamma and

// iota_upper

lambda_o_upper = PI - iota_upper - gamma;

// for cases where the sum of iota_upper and gamma are greater than

// 180 degrees a sufficiently small value is assigned for

// lambda_o_upper

if (lambda_o_upper $<0$ )

lambda_o_upper $=0.0001$;

// The length of stem imaged above the basepoint is calculated

// by the law of sines using iota_upper and lambda_o_upper as

$/ /$ angles and the image distance as a leg

$\mathrm{h} \_\mathrm{o}=(\sin ($ iota_upper $) *$ image[i].distance) $/ \sin ($ lambda_o_upper $)$;

// h_o added to the initial base height results in the greatest

// object space height captured on the image

image[i].upperh = h_o + image[i].baseht;

// iota_lower is the difference between ANG and mu

iota_lower = ANG - mu;

// cgamma is a temporary variable defined as the supplement of gamma

cgamma = PI - gamma;

// lambda_o_lower is 180 degrees minus the sum of the other angles 


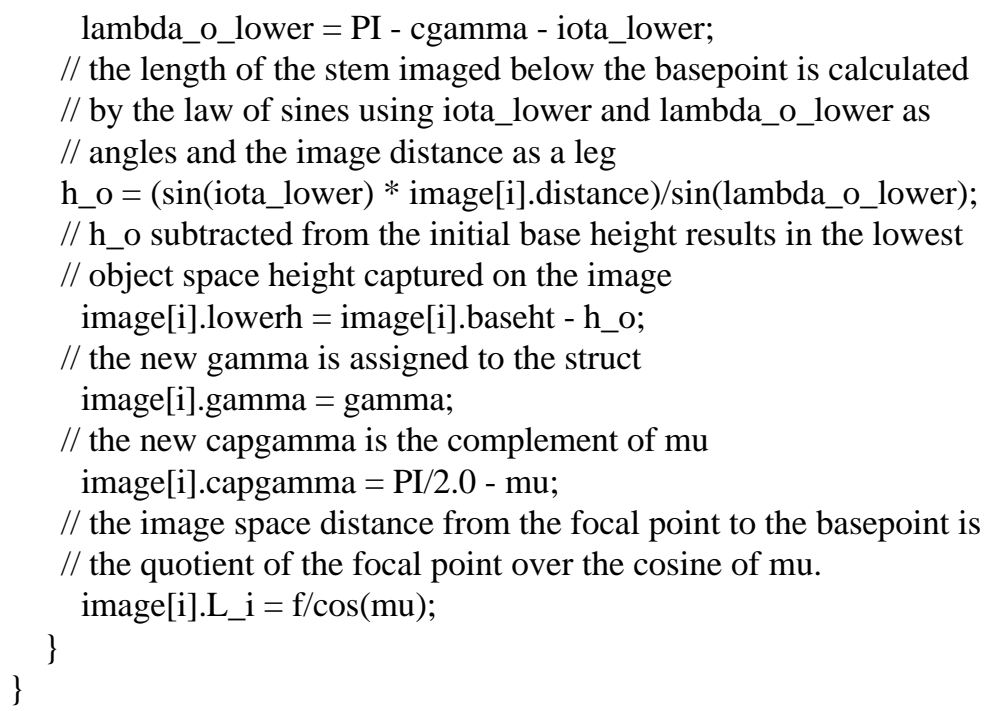

// This function takes the image_rec structure as an arguement after

// an input image file has been read. This function also allows

// interactive user input via keyboard/screen I/O in order to "register"

// upper and lower images.

void calcheights (image_rec image[], int\& num_images, char file[])

\{

// declare and initialize local variables

// selected_l = lower image number selected by search algorithm

$/ / \mathrm{c}=$ counter

// picnum_l = lower image number input by user via keyboard

// picnum_u = upper image number input by user via keyboard

// y_in_u = y value of upper image input by user via keyboard

// y_in_l = y value of lower image input by user via keyboard

// selected_u = upper image number input by user via keyboard

$/ / \mathrm{a}$ and $\mathrm{b}=$ variables used for Boolean test

$/ / \mathrm{bht}=$ user defined baseheight entered via keyboard

$/ /$ length = variable to temporarily store string length

// imlength = image space distance on lower image between

$/ / \quad$ base y value and user input $y_{-}$in_l

// G_i = image space distance between focal point and image point Bi

// G_o = object space distance between focal point and point Bo

// lambda_o = object space angle having plumb line within datum plane

// as one leg and line from an object space point and the focal point

$/ /$ iota $=$ angle with $\mathrm{Bi}, \mathrm{y} \_$in_l, and the focal point as vertices

// height_o = object space height corresponding to y_in_1

$/ /$ fileout = string array for holding filename

$/ /$ suffix = string array to append to create new filename

$/ /$ more $=$ user input character variable

$/ /$ response $=$ user input character variable

int selected $\_l=0, \mathrm{c}$, picnum $\_\mathrm{l}=0$, picnum $\_\mathrm{u}=0, \mathrm{y} \_$in $\_\mathrm{u}=0, \mathrm{y} \_$in $\_\mathrm{l}=0$, selected_u $=0, a=0, b=0$, bht, length;

double imlength, G_i, G_o, lambda_o, iota, height_o;

char fileout[85], suffix[9] = "_out.txt", more, response;

// check to see if input data file has been read 
if (image[0].imagenum $==0$ )

\{

// if no input data file, print message and run input data file function

cout $<<$ "You must read image input file first" < < endl;

readData (image, num_images, basefile);

\}

// initial check to inquire whether user wishes to edit data

cout $<<$ "Do you wish to make any changes $(\mathrm{y} / \mathrm{n}) "<<$ endl;

cin $>$ response;

switch (response) \{

// if the user enters the character ' $y$ ' then they are prompted to enter four integers case ' $y$ ' :

cout $<<$ "Enter lower picture number: ";

cin $\gg$ picnum_l;

cout $\ll<$ "Enter upper picture number: ";

cin $\gg>$ picnum_u;

cout $<<$ "Enter lower picture y-value: ";

cin $>$ y_in_l;

cout $<<$ "Enter upper picture y-value: ";

cin $>$ y_in_u;

// initialize variables to be used in search algorithm

int notfound $=1$;

$\mathrm{c}=0$

$\mathrm{a}=0$

$\mathrm{b}=0$;

// loop until both images are found or until all image records have been searched while (notfound \&\& c < num_images)

\{

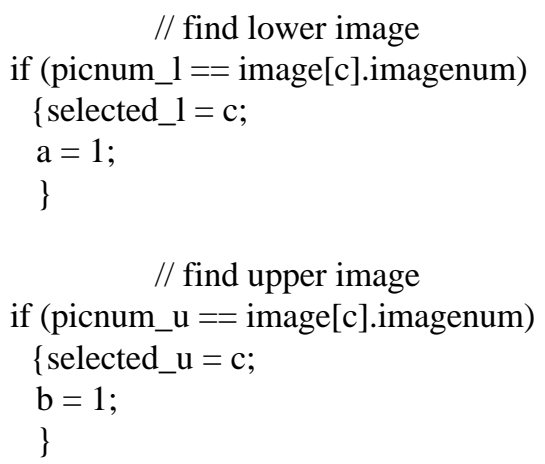

// find upper image

if (picnum_u $==$ image[c].imagenum)

$\{$ selected_u $=\mathrm{c}$;

$\mathrm{b}=1$;

\}

// increment $\mathrm{c}$ to examine next image number

else $++\mathrm{c}$;

// if upper and lower are found then assign value of 0 to notfound

// thus exiting the loop

if $(a+b==2)$

notfound $=0$; 
// only run this section if image was found if (picnum_l $>0$ )

\{

// imlength calculated from the difference between the selected images base y value and the user input y value

// for the lower image multiplied by the pixel dimension imlength $=$ double(image[selected_l].basey - y_in_l $) *$ pixdim;

$/ / G \_i$ is calculted using law of cosines using imlength and the selected image distance from focal point to base

// point $(\mathrm{Bi})$ as sides and the capgamma of the selected image as the angle.

G_i $=\operatorname{sqrt}($ pow $(i m l e n g t h, 2)+\operatorname{pow}($ image[selected_1].L_i, 2$)-2 *$ imlength $*$ image[selected_l].L_i $*$ cos(image[selected_l].capgamma));

G_i as sides

// calculate iota from law of sines using capgamma from the selected image as the angle and imlength and iota $=\operatorname{asin}\left(\sin \left(\right.\right.$ image$[$ selected_l].capgamma $) *$ imlength $\left./ \mathrm{G} \_\mathrm{i}\right)$;

// lambda_o calculated by subtracting the sum of iota and the gamma of the selected image from 180 degrees

$$
\text { lambda_o = PI - (iota + image[selected_1].gamma); }
$$

// height_o can then be calculated by adding the object space stem length from the reference point to the $/ /$ desired height to the base height height_o $=\sin ($ iota $) *$ image[selected_l].distance / sin(lambda_o)+image[selected_1].baseht;

// G_o is then calculated from law of sines using gamma from the selected image and lambda_o as angles and the

// selected image distance as the side

G_o $=\sin ($ image[selected_l].gamma) * image[selected_l].distance / $\sin ($ lambda_o);

// values for the upper image assigned from calculated values.

// the base height of the upper image is height_o

// the distance for the upper image is G_o

// the base y value is y_in_u

image[selected_u].baseht $=$ int $($ height_o);

image[selected_u].distance $=$ G_o;

image[selected_u].basey $=\mathrm{y} \_$in_u;

// prompt user to input values for lower image, this should probably have been done

// previous calculations, but correct calculation will take place regardless, because

$/ /$ of recursion below

cout $<<$ "Do you want to enter base height and yval for lower image? $(\mathrm{y} / \mathrm{n}) "$;

cin $>$ response;

// if the user enters the character 'y' then they are prompted to enter the base height

$/ /$ and y value for the lower image.

if (response $==-y$ ')

\{

cout $<<$ "Base height = (integer)"; 


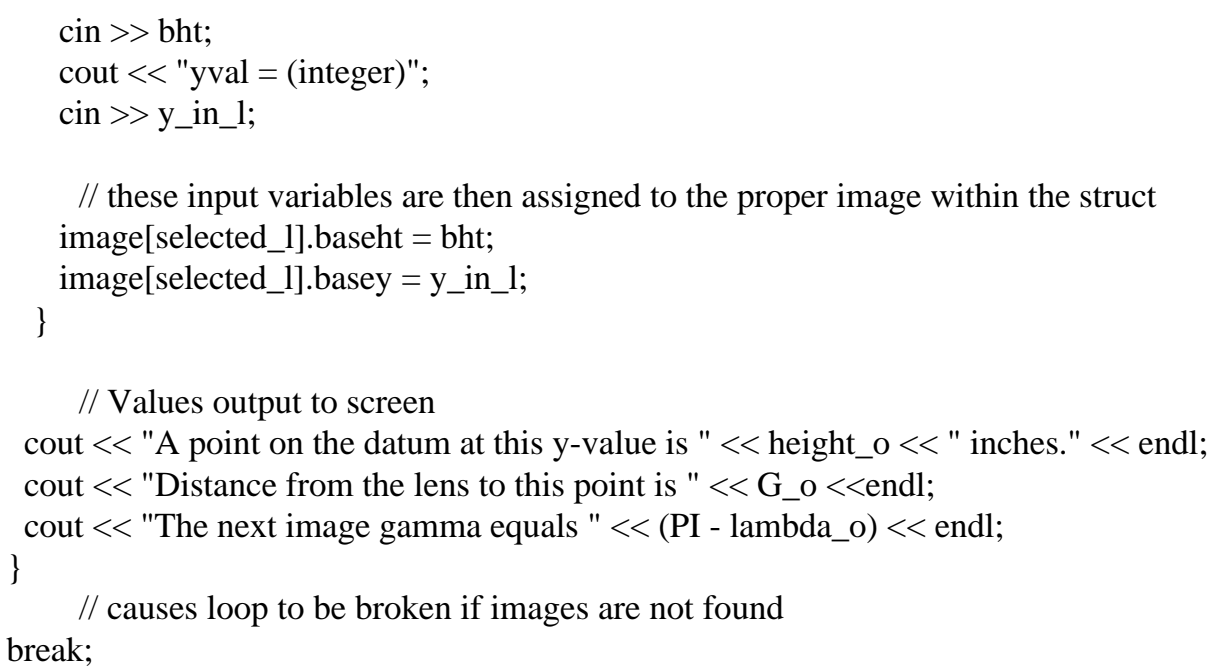

// ask user if they want to do another image, view the current values, or exit cout $\ll$ "Press [n] when ready for next image," $<$ endl

$$
<" \quad[\text { p] for current values or" } \quad<\text { endl }
$$

$<$ [d] when you're done" $<<$ endl;

$/ /$ read input

cin $>$ response;

switch (response) \{

// if they wish to enter another image run section function and calcheights // to calculate correct values

case ' $n$ ' : $\quad$ section (image, num_images);

break; calcheights (image,num_images, file);

// if they wish to view the current values loop through each record in groups of 20 // and output to the screen the image number, tree number, side number, nominal // distance, distance, base y value, and base height.

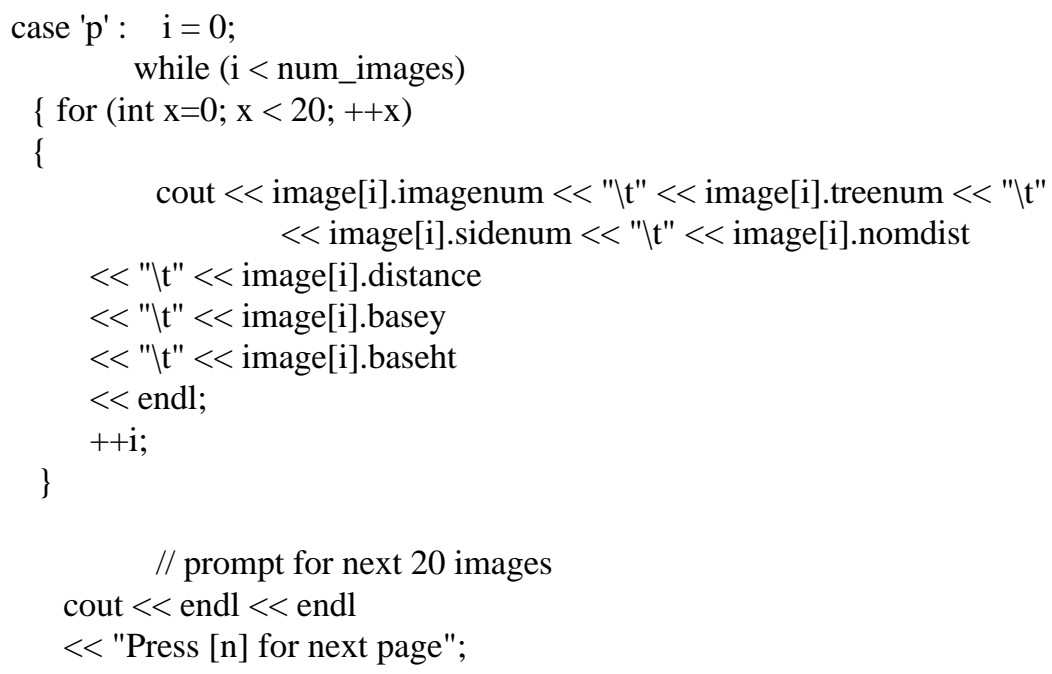

$/ /$ prompt for next 20 images

cout $<<$ endl $<<$ endl

$<<$ "Press [n] for next page"; 
cin $>>$ more;

\}

// run section and calcheights fuctions again to calculate correct values

section (image, num_images);

calcheights (image,num_images, file);

break;

// if the user enters the character 'd', an output file is created with the same name // as the input file, but with an_out.txt extension.

case ' $d$ ' : ofstream outfile; // create outfile stream

strcpy(fileout, basefile); // copy filename from global

if (!fileout) // if there is no input file then an error

$/ /$ message is shown $\{* *$ note this was

$/ /$ merged from a separate module and

$/ /$ is probably no longer needed since there

// was a similar error check earlier on in

// the function

\{

cout $<<$ "You must read image input file first" < < endl;

readData (image, num_images, basefile);

section (image, num_images);

\}

// assign new file name

length $=$ strlen (fileout) -4 ;

for $(\mathrm{c}=0 ; \mathrm{c}<$ length; $++\mathrm{c})$

fileout $[\mathrm{c}]=$ fileout $[\mathrm{c}]$;

int $\mathrm{x}=0$;

for $(; \mathrm{c}<85 ;++\mathrm{c})$

\{

fileout $[\mathrm{c}]=\operatorname{suffix}[\mathrm{x}]$;

$++\mathrm{x}$;

\}

outfile.open(fileout); // creates output file

outfile.setf(ios::floatfield); // functions used to output decimal point

outfile.setf(ios::showpoint); // and desired precision

for $(\mathrm{i}=0 ; \mathrm{i}<$ num_images; $++\mathrm{i}) / /$ for all image records

// write output to file

\{

outfile $<<$ image[i].imagenum $<<$ "\t" $<<$ image[i].treenum $<<$ "\t"

$<$ image[i].sidenum $<<$ "|t" $<<$ image[i].nomdist $<<$ "|t"

$<\operatorname{setw}(12)<<$ setprecision $(6)<<$ image[i].distance

$<$ "ไt" $<<\operatorname{setw}(22)<<$ setprecision $(16)<<$ image[i].angle

$<<$ "ไt" $<<$ image[i].basey

$<<$ "It" $<<$ image[i].baseht

$<$ "\t" < setw(22) < s setprecision (16) < image[i].upperh

$<$ "\t" $<<\operatorname{setw}(22)<<$ setprecision $(16)<<$ image[i].lowerh

$<$ "\t" $<$ setw $(22)<$ setprecision $(16)<$ image[i].gamma

$<$ "\t" $<$ setw $(22)<$ setprecision $(16)<$ image[i].capgamma 


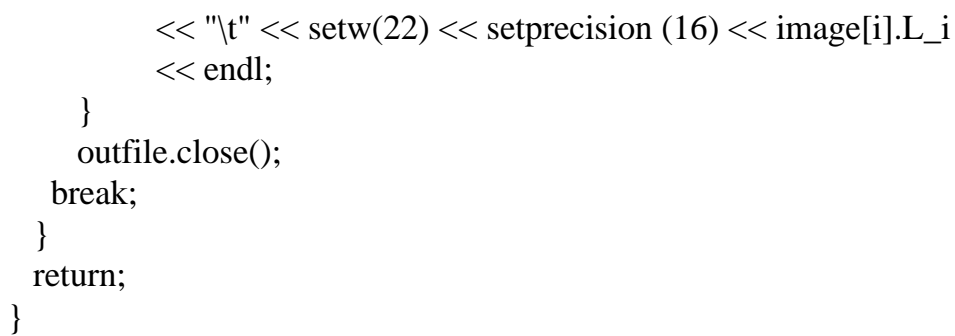

// this function is used to read in user keyboard input and pass the file value back to // the function call void getfile (char file[])

\{

// initialize string for input file char infile[80];

// read keyboard input

cin $>$ infile;

// If keyboard input is the character ' $n$ ' then the function is exited if (infile $==" n "$ ) return;

// test to see if the file exists

ifstream test;

test.open(infile, ios::nocreate);

if (test.fail())

// if the file does not exist, prompt user for new filename

\{

cout $\quad<<$ "File does not exist or cannot be opened." $<<$ endl $<<$ "try another? Enter [n] to return to menu."

getfile(file); $<$ endl;

\}

// if file does exist, close file, pass filename

else

\{test.close();

strcpy (file, infile);

\}

return;

\}

// This function reads another data file containing the tree number (int) and // height (double) and places these values into two parallel arrays,

$/ /$ it also tallies the number of records in the file and passes this value by // reference.

//

void heights_in (int tree[],double height[], int\& num_heights)

// declare and initialize local variables

$/ /$ tnum $=$ tree number

// Index = counter

$/ / \mathrm{ht}=$ height 


\section{$/ /$ heightin $=$ input tree/height file name}

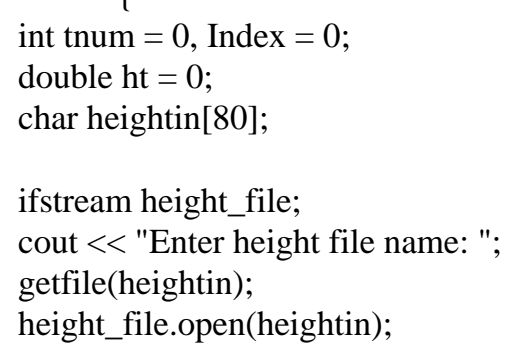

// The findimages function takes the image_rec struct and the parallel arrays // tree and height as well as a PBR integer for the number of heights.

// The function then loops through the parallel arrays of trees and heights and // finds all of the images that contain that tree and height. It also calculates

// the image row value of that height for each image and outputs that to a data // file. void findimages (image_rec image[], int tree[],double height[], int\& num_heights)

// Declare and initialize local variables

// $\quad$ c and $\mathrm{x}=$ counters

// $\quad$ length = variable to set size of string array

// heightin = height of diameter measurement in inches

// section_length = length of stem between point of measurement and base height

// $\quad$ iota = angular measurement between legs formed by the focal point rays

// $\quad$ pointing to the base point and to the height of the desired diameter measurement

// lambda_i = image space angle with principal axis as one leg and ray

$/ /$

/I extending from image point at desired diameter and passing through the focal point as the other leg 
// $\quad$ h_i_pixels = image space distance in pixels

// $\quad$ yval $=\mathrm{y}$ value of desired height

// $\quad$ G_o = object space distance between focal point and height of desired diameter

// $\quad$ suffix $=$ character array specifying a file name suffix

// fileout $=$ character array to hold the output filename

int $\mathrm{c}, \mathrm{x}$, length;

double heightin, section_length, iota,lambda_i, h_i;

double h_i_pixels, yval;

double G_o;

char suffix[9] = ".yvl.txt";

char fileout[85];

ofstream yval_file;

// create outfile stream

strcpy(fileout, basefile); // copy filename from global

if (!fileout) // error message if input file has not yet

function called in

// order to read the input file, and then

// the section function is called to ensure

// that all calculations are correct

\{

cout $<<$ "You must read image input file first" < < endl;

readData (image, num_images, basefile);

section (image, num_images);

\}

length = strlen (fileout)-4; // subtract last four characters from input

for $(\mathrm{c}=0 ; \mathrm{c}<$ length $;++\mathrm{c})$

fileout $[\mathrm{c}]=$ fileout $[\mathrm{c}]$;

$\mathrm{x}=0$;

for $(; \mathrm{c}<85 ;++\mathrm{c}) \quad / /$ append new suffix to filename

\{

fileout $[\mathrm{c}]=\operatorname{suffix}[\mathrm{x}]$;

$++\mathrm{x}$

\}

strcpy (diamfile, fileout);

yval_file.open(fileout); // open the output file

// first line of output to label values

yval_file $<<$ "image number" $<<$ "|t" $<<$ "|t" $<<$ "tree" $<<$ "|t" $\quad<<$ "side"

$<<$ "left-y"

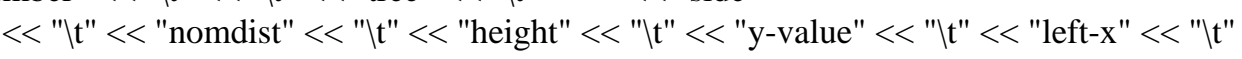

<< "lt" < < "right-x" < < "tt" < < "right-y" < < endl;

// for each tree/height record output the image number and y-value

$/ /$ corresponding to the tree and height inputs

for $(\mathrm{x}=0 ; \mathrm{x}<$ num_heights; $++\mathrm{x}) \quad$ // for every tree/height record

\{

$\mathrm{c}=0$;

// continue the loop while the tree array variable is greater than the image tree number

$/ / * * * * * \quad$ THIS REQUIRES THAT THE IMAGE TREE NUMBERS ARE IN ASCENDING ORDER 


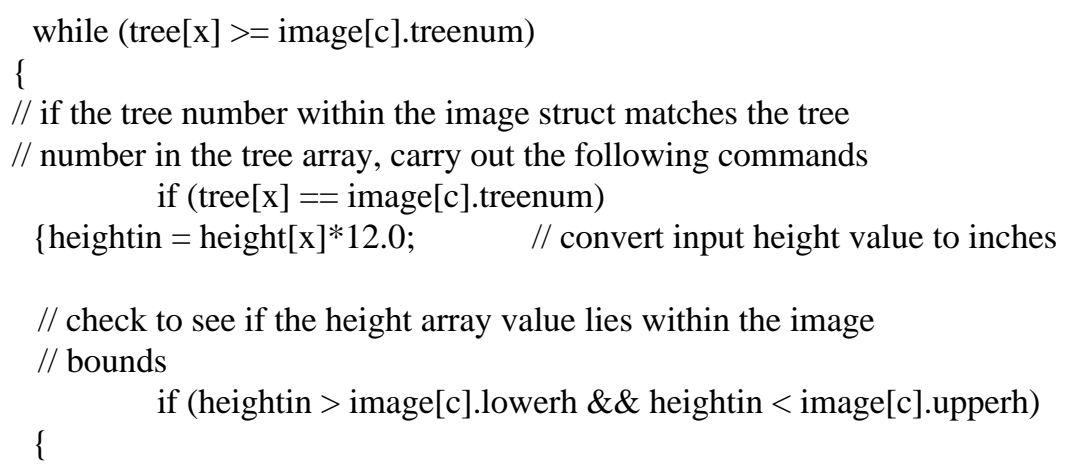

$/ /$ if it is in the image output the image number and the face $/ /$ number to the screen

cout $<<$ image[c].imagenum $<$ " face \#" < < image[c].sidenum;

// calculate the section length between the height entered and the

// base height by subtracting the base height from the height of

// the desired diameter

section_length = heightin - image[c].baseht;

// Calculate the distance from the focal point to the point on

// the datum at the desired height using law of cosines with

// the image distance (from focal point to base height from the

$/ /$ struct $\})$ and the section length as sides and gamma as the angle

G_o $=\operatorname{sqrt}($ pow $($ section_length,2) + pow $($ image[c].distance, 2$)-2 *$ section_length * image[c].distance * $\cos ($ image $[\mathrm{c}]$.gamma));

// calculate iota from law of sines using gamma from the selected

// image as the angle and section length and G_o as sides

iota $=$ asin $\left(\right.$ section_length $* \sin ($ image $[\mathrm{c}]$.gamma $\left.) / \mathrm{G} \_0\right)$;

// calculate the lambda_i by subtraction the sum of capgamma and

// iota from 180 degrees.

lambda_i = PI - image[c].capgamma - iota;

// calculate image distance representing the section length using

// law of sines with iota and lambda_i as angles and the distance

// from the focal point to the the base image point (Bi) as the

// side

h_i $=\sin ($ iota $) *$ image $[\mathrm{c}] . L \_\mathrm{i} / \sin ($ lambda_i);

// convert distance from microns to pixels

h_i_pixels $=$ h_i / pixdim;

// The base y value minus the image distance in pixels results

$/ /$ in the y value that corresponds to the desired diameter.

yval = image[c].basey - h_i_pixels;

// output the y value to the screen

cout $<$ " y-value " < y yal < endl;

// output the image number, tree number, side number, nominal

// distance, height and y value to output file 


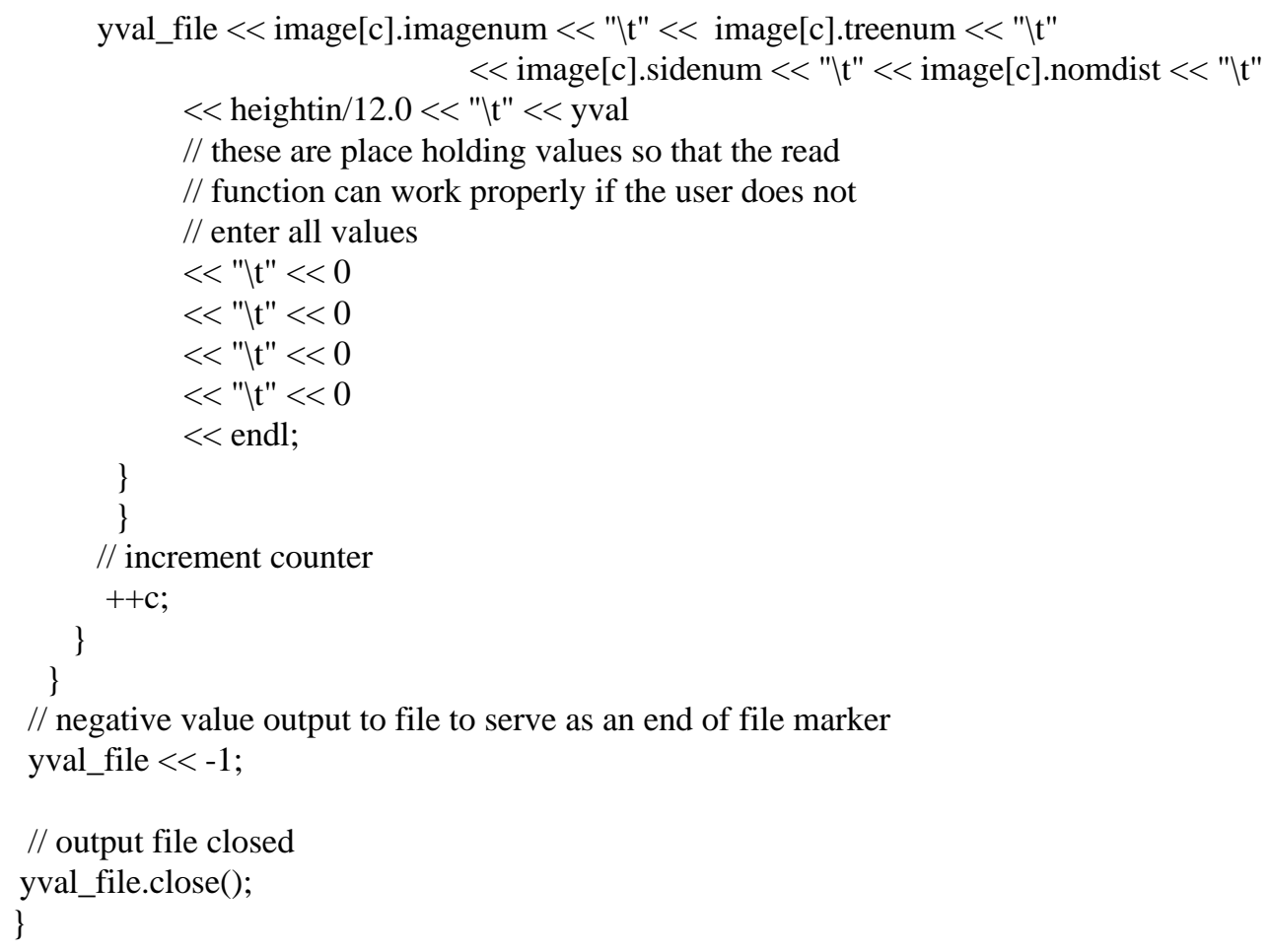

// this function takes the image_rec structure and an character array // containing the input filename (should be the .yvl.txt file that was $/ /$ produced in the findimages function and modified to contain the // correct lx, ly, rx, ry values) as arguements. It reads values from the // struct and calculates the diameters for each record. \{ void diam_calc(image_rec image[], char file[])

//declare local variables.

// $\quad \mathrm{i}=$ counter

// tnum, snum, lx, ly, rx, ry, imgname, nomdist, height, and ii_y are local

// variables that correspond to values in the input file

// length = variable to define string length

// lambda_o = object space angle having plumb line within datum plane

// as one leg and line from an object space point to the focal point

// as the second leg

// $\quad$ iota $=$ angular measurement between legs formed by the focal point rays

// $\quad$ pointing to the base point and to the height of the desired diameter measurement

// $\quad \mathrm{a}, \mathrm{b}$, and $\mathrm{c}=$ image space distances that form a triangle with vertices

// of the focal point and two image points representing the diameter

// $\quad$ tempdist = temporary storage variable

// $\quad \mathrm{s}=$ one half of the perimeter of triamge $\mathrm{abc}$

// phi $=$ angle between two rays from focal point to two diameter points

// G_o = object space distance between the focal point of the camera and

// the midpoint between the diameter points at datum

// $\quad$ xoff $=$ offset corrective distance to correct for perpendicular stem lean

// look = temporary storage variable

// $\quad$ mpx, mpy = image space midpoint coordinates used to calculate

// $\quad$ perpendicular stem lean

// $\quad$ diameter $=$ calculated diameter 
$/ / \quad$ filein and fileout $=$ string arrays to hold filenames

// $\quad$ suffix = string array containing output file suffix

int $\mathrm{i}$, tnum $=0$, snum=0,lx, ly, rx, ry, nomdist, length;

double lambda_o, iota, a, b, c, tempdist,s,phi, G_o,xoff, look;

double height $=0$,ii_y, mpx, mpy, diameter;

int imgname $=0$;

char filein[85], fileout[85], suffix[10] = ".diam.txt";

$\begin{array}{lc}\begin{array}{l}\text { ifstream yfile; } \\ \text { yfile.open(file); }\end{array} & \text { // create infile stream } \\ & / / \text { open input file } \\ \text { ofstream d_out; } & / / \text { create outfile stream } \\ \text { strcpy(fileout, file); } & / / \text { copy filename to fileout array } \\ \text { if (!fileout) } & / / \text { if fileout does not exist send them back }\end{array}$

// to the menu to find images and output row

vals

\{

cout $<<$ "You must find images and output row values first" $<<$ endl;

return;

\}

length $=$ strlen(fileout)-9; $\quad / /$ cut old suffix off of filename

for $(\mathrm{i}=0 ; \mathrm{i}<$ length; ++i)

fileout $[\mathrm{i}]=$ fileout $[\mathrm{i}]$;

int $\mathrm{x}=0$;

for $(; \mathrm{i}<85 ;++\mathrm{i})$

\{

fileout $[\mathrm{i}]=\operatorname{suffix}[\mathrm{x}] ; \quad$ // put new suffix on output filename

$++\mathrm{x}$

\}

d_out.open(fileout); $\quad$ // open output file

if (image[0].imagenum $==0$ ) // check to make sure input file has been read

function

\{

cout $<<$ "You must read image input file first" $<<$ endl;

readData (image, num_images, basefile);

section (image, num_images);

\}

\section{//PRIME READ}

yfile $>>$ imgname $>>$ tnum $>$ snum $>$ nomdist $>>$ height $>>$ ii_y $>>1 x>>$ ly $>>$ rx $>>$ ry;

// initialize counter

$\mathrm{i}=0$;

$/ /$ perform for all records in file

while (yfile)

\{

// continue search ORDERED struct imagenumbers until correct record is found

$/ / * * * * * \quad$ THIS REQUIRES THAT THE IMAGE NUMBERS WITHIN THE STRUCT ARE IN ASCENDING ORDER 
while (image[i].imagenum $<=$ imgname)

\{

// if the image numbers match perform the calculate the diameter

if (image[i].imagenum $==$ imgname)

\{

// calculate midpoint coordinates

$\operatorname{mpx}=$ double $((\mathrm{lx}+\mathrm{rx}) / 2.0)$;

mpy = double $((\mathrm{ly}+\mathrm{ry}) / 2.0)$;

// calculate distances for the computation of phi

tempdist $=\operatorname{sqrt}(\operatorname{pow}((480-1 \mathrm{x}) * \operatorname{pixdim}, 2)+\operatorname{pow}((640-1 \mathrm{y}) * \operatorname{pixdim}, 2))$;

// distance from focal point to left diameter point coordinate

$\mathrm{a}=\operatorname{sqrt}(\operatorname{pow}(\mathrm{f}, 2)+\operatorname{pow}($ tempdist, 2$))$;

tempdist $=\operatorname{sqrt}(\operatorname{pow}((480-\mathrm{rx}) * \operatorname{pixdim}, 2)+\operatorname{pow}((640-\mathrm{ry}) * \operatorname{pixdim}, 2))$;

// distance from focal point to right diameter point coordinate

$\mathrm{b}=\operatorname{sqrt}(\operatorname{pow}(\mathrm{f}, 2)+\operatorname{pow}($ tempdist, 2$))$;

tempdist $=\operatorname{sqrt}(\operatorname{pow}((\operatorname{rx}-1 \mathrm{x}-1) * \operatorname{pixdim}, 2)+\operatorname{pow}((\mathrm{ry}-\mathrm{ly}) * \operatorname{pixdim}, 2))$;

// distance between the left and right diameter points on image plane

$\mathrm{c}=$ tempdist;

// calculate phi from oblique triangle formula using three known sides

$\mathrm{s}=0.5 *(\mathrm{a}+\mathrm{b}+\mathrm{c})$;

look $=\operatorname{sqrt}(s *(s-a) *(s-b) *(s-c))$;

phi $=2 * \operatorname{look} /(\mathrm{a} * \mathrm{~b})$;

phi $=\operatorname{asin}($ phi $)$;

// calculate object space angle between basepoint and diameter height iota $=\operatorname{asin}(\sin ($ image$[i] . c a p g a m m a) *((i m a g e[i] . b a s e y-m p y) *$ pixdim $) \quad /$ image[i].L_i);

// calculate complement of declination angle from diameter height lambda_o = PI - iota - image[i].gamma;

// calculate distance from diameter height at datum to focal point G_o $=(\sin ($ image $[i]$.gamma $) *$ image $[i]$.distance/sin $($ lambda_o $))$;

// adjust for $\mathrm{x}$ offset

tempdist $=\operatorname{sqrt}(\operatorname{pow}(480-\mathrm{mpx}, 2)+\operatorname{pow}(640-\mathrm{mpy}, 2))$;

$\mathrm{a}=\operatorname{sqrt}(\operatorname{pow}(\mathrm{f}, 2)+\operatorname{pow}($ tempdist, 2$))$;

$\mathrm{b}=\operatorname{sqrt}(\operatorname{pow}(\mathrm{f}, 2)+\operatorname{pow}(640-\mathrm{mpy}, 2))$;

xoff $=a \cos (b / a)$;

$\mathrm{G} \_\mathrm{o}=\mathrm{G} \_\mathrm{o} / \cos (\mathrm{xoff})$;

// calculate diameter

diameter $=2.0 *(\sin (\mathrm{phi} / 2.0)) * \mathrm{G}_{-} \mathrm{o}$;

// output 0 for value if no coordinates available if $(\mathrm{lx}==0)$

diameter $=0$;

// output values to file

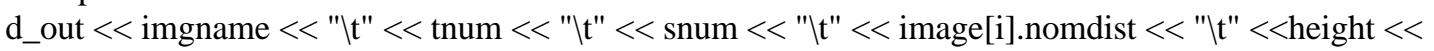


"It" < < diameter $<<$ "|t" << G_o $<<" \mid t " ~<<$ phi $<<$ endl;

$/ /$ read next record

yfile $>$ imgname $>>$ tnum $>>$ snum $>>$ nomdist $>$ height $>$ ii_y $>$ > lx $>$ ly $>$ rx $>$ ry;

// decrement counter for next record search

// To avoid the need of having the struct ordered this can be set equal

// to zero each time and the while loop can sequentially search through

$/ /$ all of the records

$\mathrm{i}-=1$;

\}

$++\mathrm{i} ; \quad$ // increment counter to compare next record

\}

\}

yfile.close();

d_out.close();

// close input file

// close output file

\} 


\section{Appendix C - SAS files}

\section{INPUT DATA}

options nodate pageno $=1$;

data tm;

input tree nomdiam nomdist height cam cal diff; datalines;

$\begin{array}{lllllll}8 & 1 & 3.05 & 1.37 & 15.49 & 16.51 & -1.02 \\ 3 & 1 & 3.05 & 1.37 & 20.83 & 19.56 & 1.27 \\ 9 & 1 & 3.05 & 1.37 & 21.84 & 21.84 & 0 \\ 18 & 1 & 3.05 & 1.37 & 26.92 & 26.42 & 0.51 \\ 10 & 1 & 3.05 & 1.37 & 28.7 & 27.69 & 1.02 \\ 8 & 1 & 3.05 & 2.44 & 14.99 & 15.75 & -0.76 \\ 3 & 1 & 3.05 & 2.44 & 17.78 & 18.54 & -0.76 \\ 9 & 1 & 3.05 & 2.44 & 19.3 & 19.56 & -0.25 \\ 18 & 1 & 3.05 & 2.44 & 23.88 & 25.15 & -1.27 \\ 10 & 1 & 3.05 & 2.44 & 25.4 & 25.65 & -0.25 \\ 8 & 1 & 3.05 & 3.66 & 13.46 & 15.24 & -1.78 \\ 3 & 1 & 3.05 & 3.66 & 17.02 & 18.03 & -1.02 \\ 9 & 1 & 3.05 & 3.66 & 18.03 & 19.05 & -1.02 \\ 18 & 1 & 3.05 & 3.66 & 23.62 & 23.88 & -0.25 \\ 10 & 1 & 3.05 & 3.66 & 23.88 & 24.38 & -0.51 \\ 8 & 1 & 3.05 & 4.88 & 13.46 & 13.72 & -0.25 \\ 3 & 1 & 3.05 & 4.88 & 17.53 & 17.53 & 0 \\ 9 & 1 & 3.05 & 4.88 & 17.27 & 17.78 & -0.51 \\ 18 & 1 & 3.05 & 4.88 & 21.84 & 24.38 & -2.54 \\ 10 & 1 & 3.05 & 4.88 & 22.86 & 24.64 & -1.78 \\ 8 & 1 & 3.05 & 6.1 & 11.68 & 13.46 & -1.78 \\ 3 & 1 & 3.05 & 6.1 & 17.53 & 17.27 & 0.25 \\ 18 & 1 & 3.05 & 6.1 & 21.08 & 22.35 & -1.27 \\ 10 & 1 & 3.05 & 6.1 & 23.88 & 24.64 & -0.76 \\ 8 & 1 & 3.05 & 7.32 & 12.45 & 12.7 & -0.25 \\ 3 & 1 & 3.05 & 7.32 & 15.49 & 15.75 & -0.25 \\ 18 & 1 & 3.05 & 7.32 & 21.84 & 21.08 & 0.76 \\ 10 & 1 & 3.05 & 7.32 & 21.84 & 23.11 & -1.27 \\ 8 & 1 & 3.05 & 8.53 & 10.16 & 11.68 & -1.52 \\ 3 & 1 & 3.05 & 8.53 & 14.99 & 15.24 & -0.25 \\ 18 & 1 & 3.05 & 8.53 & 19.56 & 20.07 & -0.51 \\ 10 & 1 & 3.05 & 8.53 & 21.84 & 22.61 & -0.76 \\ 3 & 1 & 3.05 & 9.75 & 13.72 & 14.22 & -0.51 \\ 9 & 1 & 3.05 & 9.75 & 13.46 & 14.73 & -1.27 \\ 10 & 1 & 3.05 & 9.75 & 20.32 & 20.32 & 0 \\ 3 & 1 & 3.05 & 10.97 & 13.46 & 13.46 & 0 \\ 9 & 1 & 3.05 & 10.97 & 12.95 & 13.46 & -0.51 \\ 10 & 1 & 3.05 & 10.97 & 17.27 & 19.05 & -1.78 \\ 3 & 1 & 3.05 & 12.19 & 12.95 & 13.21 & -0.25 \\ 8 & 1 & 6.1 & 1.37 & 16.26 & 16.51 & -0.25 \\ 3 & 1 & 6.1 & 1.37 & 19.56 & 19.56 & 0 \\ 9 & 1 & 6.1 & 1.37 & 22.35 & 22.35 & 0 \\ 18 & 1 & 6.1 & 1.37 & 26.67 & 26.42 & 0.25 \\ 10 & 1 & 6.1 & 1.37 & 27.18 & 27.69 & -0.51 \\ 8 & 1 & 6.1 & 2.44 & 14.99 & 15.75 & -0.76\end{array}$




$\begin{array}{lllllll}3 & 1 & 6.1 & 2.44 & 18.8 & 18.54 & 0.25 \\ 9 & 1 & 6.1 & 2.44 & 19.81 & 19.81 & 0 \\ 18 & 1 & 6.1 & 2.44 & 25.15 & 25.15 & 0 \\ 10 & 1 & 6.1 & 2.44 & 25.65 & 25.65 & 0 \\ 8 & 1 & 6.1 & 3.66 & 13.72 & 15.24 & -1.52 \\ 3 & 1 & 6.1 & 3.66 & 17.78 & 18.03 & -0.25 \\ 9 & 1 & 6.1 & 3.66 & 19.3 & 19.56 & -0.25 \\ 18 & 1 & 6.1 & 3.66 & 23.88 & 23.88 & 0 \\ 10 & 1 & 6.1 & 3.66 & 23.62 & 24.38 & -0.76 \\ 8 & 1 & 6.1 & 4.88 & 12.7 & 13.72 & -1.02 \\ 3 & 1 & 6.1 & 4.88 & 16.76 & 17.53 & -0.76 \\ 9 & 1 & 6.1 & 4.88 & 19.05 & 18.29 & 0.76 \\ 10 & 1 & 6.1 & 4.88 & 23.88 & 24.64 & -0.76 \\ 8 & 1 & 6.1 & 6.1 & 11.68 & 13.46 & -1.78 \\ 3 & 1 & 6.1 & 6.1 & 16.51 & 17.27 & -0.76 \\ 9 & 1 & 6.1 & 6.1 & 17.78 & 17.78 & 0 \\ 10 & 1 & 6.1 & 6.1 & 24.13 & 24.64 & -0.51 \\ 8 & 1 & 6.1 & 7.32 & 12.19 & 12.7 & -0.51 \\ 3 & 1 & 6.1 & 7.32 & 14.99 & 15.75 & -0.76 \\ 9 & 1 & 6.1 & 7.32 & 16.76 & 16.76 & 0 \\ 10 & 1 & 6.1 & 7.32 & 21.34 & 23.11 & -1.78 \\ 8 & 1 & 6.1 & 8.53 & 10.16 & 11.68 & -1.52 \\ 3 & 1 & 6.1 & 8.53 & 14.73 & 15.24 & -0.51 \\ 9 & 1 & 6.1 & 8.53 & 15.75 & 16 & -0.25 \\ 10 & 1 & 6.1 & 8.53 & 22.1 & 22.61 & -0.51 \\ 8 & 1 & 6.1 & 9.75 & 9.14 & 10.41 & -1.27 \\ 3 & 1 & 6.1 & 9.75 & 12.95 & 14.22 & -1.27 \\ 9 & 1 & 6.1 & 9.75 & 15.49 & 14.73 & 0.76 \\ 10 & 1 & 6.1 & 9.75 & 19.05 & 20.32 & -1.27 \\ 3 & 1 & 6.1 & 10.97 & 13.21 & 13.46 & -0.25 \\ 9 & 1 & 6.1 & 10.97 & 13.21 & 13.72 & -0.51 \\ 18 & 1 & 6.1 & 10.97 & 20.83 & 17.27 & 3.56 \\ 10 & 1 & 6.1 & 10.97 & 18.54 & 19.05 & -0.51 \\ 3 & 1 & 6.1 & 12.19 & 11.43 & 13.21 & -1.78 \\ 9 & 1 & 6.1 & 12.19 & 13.21 & 13.46 & -0.25 \\ 18 & 1 & 6.1 & 12.19 & 16.76 & 16.51 & 0.25 \\ 9 & 1 & 6.1 & 13.41 & 11.94 & 12.19 & -0.25 \\ 8 & 1 & 9.14 & 1.37 & 18.29 & 16.51 & 1.78 \\ 3 & 1 & 9.14 & 1.37 & 19.81 & 19.56 & 0.25 \\ 9 & 1 & 9.14 & 1.37 & 21.08 & 21.34 & -0.25 \\ 18 & 1 & 9.14 & 1.37 & 26.42 & 26.42 & 0 \\ 10 & 1 & 9.14 & 1.37 & 26.67 & 27.69 & -1.02 \\ 8 & 1 & 9.14 & 2.44 & 15.49 & 15.75 & -0.25 \\ 3 & 1 & 9.14 & 2.44 & 18.8 & 18.54 & 0.25 \\ 9 & 1 & 9.14 & 2.44 & 19.81 & 19.81 & 0 \\ 18 & 1 & 9.14 & 2.44 & 24.89 & 25.15 & -0.25 \\ 10 & 1 & 9.14 & 2.44 & 25.65 & 25.65 & 0 \\ 8 & 1 & 9.14 & 3.66 & 13.46 & 15.24 & -1.78 \\ 3 & 1 & 9.14 & 3.66 & 16.76 & 18.03 & -1.27 \\ 9 & 1 & 9.14 & 3.66 & 19.56 & 20.07 & -0.51 \\ 18 & 1 & 9.14 & 3.66 & 23.88 & 23.88 & 0 \\ 10 & 1 & 9.14 & 3.66 & 23.62 & 24.38 & -0.76 \\ 8 & 1 & 9.14 & 4.88 & 12.45 & 13.72 & -1.27 \\ 3 & 1 & 9.14 & 4.88 & 16.51 & 17.53 & -1.02 \\ & & & & & & \end{array}$




$\begin{array}{lllllll}9 & 1 & 9.14 & 4.88 & 19.05 & 18.54 & 0.51 \\ 18 & 1 & 9.14 & 4.88 & 23.88 & 24.38 & -0.51 \\ 10 & 1 & 9.14 & 4.88 & 23.88 & 24.64 & -0.76 \\ 8 & 1 & 9.14 & 6.1 & 12.19 & 13.46 & -1.27 \\ 3 & 1 & 9.14 & 6.1 & 16.26 & 17.27 & -1.02 \\ 9 & 1 & 9.14 & 6.1 & 18.8 & 18.8 & 0 \\ 10 & 1 & 9.14 & 6.1 & 23.88 & 24.64 & -0.76 \\ 8 & 1 & 9.14 & 7.32 & 11.94 & 12.7 & -0.76 \\ 3 & 1 & 9.14 & 7.32 & 15.49 & 15.75 & -0.25 \\ 9 & 1 & 9.14 & 7.32 & 16.76 & 16 & 0.76 \\ 10 & 1 & 9.14 & 7.32 & 22.1 & 23.11 & -1.02 \\ 8 & 1 & 9.14 & 8.53 & 10.41 & 11.68 & -1.27 \\ 3 & 1 & 9.14 & 8.53 & 14.99 & 15.24 & -0.25 \\ 9 & 1 & 9.14 & 8.53 & 15.24 & 16 & -0.76 \\ 10 & 1 & 9.14 & 8.53 & 21.34 & 22.61 & -1.27 \\ 8 & 1 & 9.14 & 9.75 & 9.4 & 10.41 & -1.02 \\ 3 & 1 & 9.14 & 9.75 & 13.46 & 14.22 & -0.76 \\ 9 & 1 & 9.14 & 9.75 & 13.72 & 14.48 & -0.76 \\ 10 & 1 & 9.14 & 9.75 & 18.03 & 20.32 & -2.29 \\ 8 & 1 & 9.14 & 10.97 & 8.13 & 9.91 & -1.78 \\ 3 & 1 & 9.14 & 10.97 & 12.7 & 13.46 & -0.76 \\ 9 & 1 & 9.14 & 10.97 & 13.21 & 13.97 & -0.76 \\ 18 & 1 & 9.14 & 10.97 & 17.53 & 17.27 & 0.25 \\ 10 & 1 & 9.14 & 10.97 & 18.03 & 19.05 & -1.02 \\ 9 & 1 & 9.14 & 12.19 & 12.19 & 12.7 & -0.51 \\ 3 & 1 & 9.14 & 12.19 & 12.19 & 13.21 & -1.02 \\ 18 & 1 & 9.14 & 12.19 & 17.27 & 16.51 & 0.76 \\ 3 & 1 & 9.14 & 13.41 & 11.43 & 10.67 & 0.76 \\ 9 & 1 & 9.14 & 13.41 & 10.16 & 10.92 & -0.76 \\ 18 & 1 & 9.14 & 13.41 & 15.49 & 15.24 & 0.25 \\ 9 & 1 & 9.14 & 14.63 & 9.4 & 9.91 & -0.51 \\ 9 & 1 & 9.14 & 15.85 & 7.11 & 8.13 & -1.02 \\ 8 & 1 & 12.19 & 1.37 & 18.03 & 16.51 & 1.52 \\ 3 & 1 & 12.19 & 1.37 & 20.07 & 19.56 & 0.51 \\ 9 & 1 & 12.19 & 1.37 & 20.57 & 20.83 & -0.25 \\ 18 & 1 & 12.19 & 1.37 & 26.42 & 26.42 & 0 \\ 10 & 1 & 12.19 & 1.37 & 26.42 & 27.69 & -1.27 \\ 8 & 1 & 12.19 & 2.44 & 15.24 & 15.75 & -0.51 \\ 3 & 1 & 12.19 & 2.44 & 18.03 & 18.54 & -0.51 \\ 9 & 1 & 12.19 & 2.44 & 20.07 & 20.32 & -0.25 \\ 18 & 1 & 12.19 & 2.44 & 25.4 & 25.15 & 0.25 \\ 10 & 1 & 12.19 & 2.44 & 25.4 & 25.65 & -0.25 \\ 8 & 1 & 12.19 & 3.66 & 14.73 & 15.24 & -0.51 \\ 3 & 1 & 12.19 & 3.66 & 17.27 & 18.03 & -0.76 \\ 9 & 1 & 12.19 & 3.66 & 18.8 & 19.3 & -0.51 \\ 18 & 1 & 12.19 & 3.66 & 24.64 & 23.88 & 0.76 \\ 10 & 1 & 12.19 & 3.66 & 24.13 & 24.38 & -0.25 \\ 8 & 1 & 12.19 & 4.88 & 13.97 & 13.72 & 0.25 \\ 3 & 1 & 12.19 & 4.88 & 16.76 & 17.53 & -0.76 \\ 9 & 1 & 12.19 & 4.88 & 18.8 & 19.05 & -0.25 \\ 18 & 1 & 12.19 & 4.88 & 23.11 & 24.38 & -1.27 \\ 10 & 1 & 12.19 & 4.88 & 23.37 & 24.64 & -1.27 \\ 8 & 1 & 12.19 & 6.1 & 12.19 & 13.46 & -1.27 \\ 3 & 1 & 12.19 & 6.1 & 16 & 17.27 & -1.27\end{array}$




\begin{tabular}{|c|c|c|c|c|c|c|}
\hline 9 & 1 & 12.19 & 6.1 & 17.02 & 17.53 & -0.51 \\
\hline 10 & 1 & 12.19 & 6.1 & 24.13 & 24.64 & -0.51 \\
\hline 8 & 1 & 12.19 & 7.32 & 12.19 & 12.7 & -0.51 \\
\hline 3 & 1 & 12.19 & 7.32 & 15.49 & 15.75 & -0.25 \\
\hline 9 & 1 & 12.19 & 7.32 & 16.26 & 16.76 & -0.51 \\
\hline 10 & 1 & 12.19 & 7.32 & 22.35 & 23.11 & -0.76 \\
\hline 8 & 1 & 12.19 & 8.53 & 10.92 & 11.68 & -0.76 \\
\hline 3 & 1 & 12.19 & 8.53 & 13.97 & 15.24 & -1.27 \\
\hline 9 & 1 & 12.19 & 8.53 & 14.73 & 15.75 & -1.02 \\
\hline 10 & 1 & 12.19 & 8.53 & 21.34 & 22.61 & -1.27 \\
\hline 8 & 1 & 12.19 & 9.75 & 10.67 & 10.41 & 0.25 \\
\hline 3 & 1 & 12.19 & 9.75 & 13.46 & 14.22 & -0.76 \\
\hline 9 & 1 & 12.19 & 9.75 & 14.48 & 15.49 & -1.02 \\
\hline 18 & 1 & 12.19 & 9.75 & 20.83 & 19.81 & 1.02 \\
\hline 10 & 1 & 12.19 & 9.75 & 19.81 & 20.32 & -0.51 \\
\hline 8 & 1 & 12.19 & 10.97 & 9.14 & 9.91 & -0.76 \\
\hline 9 & 1 & 12.19 & 10.97 & 12.95 & 13.46 & -0.51 \\
\hline 3 & 1 & 12.19 & 10.97 & 12.19 & 13.46 & -1.27 \\
\hline 18 & 1 & 12.19 & 10.97 & 20.32 & 17.27 & 3.05 \\
\hline 10 & 1 & 12.19 & 10.97 & 18.54 & 19.05 & -0.51 \\
\hline 9 & 1 & 12.19 & 12.19 & 11.94 & 12.95 & -1.02 \\
\hline 3 & 1 & 12.19 & 12.19 & 11.94 & 13.21 & -1.27 \\
\hline 18 & 1 & 12.19 & 12.19 & 18.29 & 16.51 & 1.78 \\
\hline 10 & 1 & 12.19 & 12.19 & 18.54 & 20.57 & -2.03 \\
\hline 3 & 1 & 12.19 & 13.41 & 10.92 & 10.67 & 0.25 \\
\hline 9 & 1 & 12.19 & 13.41 & 11.94 & 12.7 & -0.76 \\
\hline 18 & 1 & 12.19 & 13.41 & 16 & 15.24 & 0.76 \\
\hline 9 & 1 & 12.19 & 14.63 & 9.91 & 10.92 & -1.02 \\
\hline 9 & 1 & 12.19 & 15.85 & 8.38 & 9.4 & -1.02 \\
\hline 8 & 1 & 15.24 & 1.37 & 19.3 & 16.51 & 2.79 \\
\hline 3 & 1 & 15.24 & 1.37 & 21.08 & 19.56 & 1.52 \\
\hline 9 & 1 & 15.24 & 1.37 & 20.57 & 20.83 & -0.25 \\
\hline 18 & 1 & 15.24 & 1.37 & 26.16 & 26.42 & -0.25 \\
\hline 10 & 1 & 15.24 & 1.37 & 26.42 & 27.69 & -1.27 \\
\hline 8 & 1 & 15.24 & 2.44 & 14.99 & 15.75 & -0.76 \\
\hline 3 & 1 & 15.24 & 2.44 & 19.56 & 18.54 & 1.02 \\
\hline 9 & 1 & 15.24 & 2.44 & 19.3 & 19.56 & -0.25 \\
\hline 18 & 1 & 15.24 & 2.44 & 25.91 & 25.15 & 0.76 \\
\hline 10 & 1 & 15.24 & 2.44 & 24.89 & 25.65 & -0.76 \\
\hline 8 & 1 & 15.24 & 3.66 & 13.21 & 15.24 & -2.03 \\
\hline 3 & 1 & 15.24 & 3.66 & 17.53 & 18.03 & -0.51 \\
\hline 9 & 1 & 15.24 & 3.66 & 18.03 & 18.54 & -0.51 \\
\hline 18 & 1 & 15.24 & 3.66 & 23.88 & 23.88 & 0 \\
\hline 10 & 1 & 15.24 & 3.66 & 22.86 & 24.38 & -1.52 \\
\hline 8 & 1 & 15.24 & 4.88 & 12.45 & 13.72 & -1.27 \\
\hline 9 & 1 & 15.24 & 4.88 & 16.76 & 17.27 & -0.51 \\
\hline 3 & 1 & 15.24 & 4.88 & 16.51 & 17.53 & -1.02 \\
\hline 18 & 1 & 15.24 & 4.88 & 23.11 & 24.38 & -1.27 \\
\hline 10 & 1 & 15.24 & 4.88 & 22.86 & 24.64 & -1.78 \\
\hline 8 & 1 & 15.24 & 6.1 & 11.94 & 13.46 & -1.52 \\
\hline 9 & 1 & 15.24 & 6.1 & 16.51 & 16.51 & 0 \\
\hline 3 & 1 & 15.24 & 6.1 & 17.02 & 17.27 & -0.25 \\
\hline 10 & 1 & 15.24 & 6.1 & 22.86 & 24.64 & -1.78 \\
\hline 8 & 1 & 15.24 & 7.32 & 12.45 & 12.7 & -0.25 \\
\hline
\end{tabular}




\begin{tabular}{|c|c|c|c|c|c|c|}
\hline 3 & 1 & 15.24 & 7.32 & 15.49 & 15.75 & -0.25 \\
\hline 9 & 1 & 15.24 & 7.32 & 16.26 & 16.76 & -0.51 \\
\hline 10 & 1 & 15.24 & 7.32 & 21.59 & 23.11 & -1.52 \\
\hline 8 & 1 & 15.24 & 8.53 & 12.19 & 11.68 & 0.51 \\
\hline 3 & 1 & 15.24 & 8.53 & 14.22 & 15.24 & -1.02 \\
\hline 9 & 1 & 15.24 & 8.53 & 15.24 & 15.49 & -0.25 \\
\hline 10 & 1 & 15.24 & 8.53 & 20.07 & 22.61 & -2.54 \\
\hline 8 & 1 & 15.24 & 9.75 & 10.16 & 10.41 & -0.25 \\
\hline 3 & 1 & 15.24 & 9.75 & 14.99 & 14.22 & 0.76 \\
\hline 9 & 1 & 15.24 & 9.75 & 14.48 & 15.24 & -0.76 \\
\hline 10 & 1 & 15.24 & 9.75 & 18.29 & 20.32 & -2.03 \\
\hline 8 & 1 & 15.24 & 10.97 & 7.87 & 9.91 & -2.03 \\
\hline 9 & 1 & 15.24 & 10.97 & 12.19 & 13.21 & -1.02 \\
\hline 3 & 1 & 15.24 & 10.97 & 13.72 & 13.46 & 0.25 \\
\hline 10 & 1 & 15.24 & 10.97 & 18.29 & 19.05 & -0.76 \\
\hline 9 & 1 & 15.24 & 12.19 & 11.68 & 12.45 & -0.76 \\
\hline 10 & 1 & 15.24 & 12.19 & 18.8 & 20.57 & -1.78 \\
\hline 9 & 1 & 15.24 & 13.41 & 10.16 & 11.18 & -1.02 \\
\hline 9 & 1 & 15.24 & 14.63 & 10.16 & 10.67 & -0.51 \\
\hline 9 & 1 & 15.24 & 15.85 & 7.62 & 8.64 & -1.02 \\
\hline 1 & 2 & 3.05 & 1.37 & 29.97 & 29.97 & 0 \\
\hline 2 & 2 & 3.05 & 1.37 & 34.29 & 33.27 & 1.02 \\
\hline 19 & 2 & 3.05 & 1.37 & 41.15 & 38.35 & 2.79 \\
\hline 13 & 2 & 3.05 & 1.37 & 39.12 & 38.35 & 0.76 \\
\hline 15 & 2 & 3.05 & 1.37 & 43.94 & 44.7 & -0.76 \\
\hline 1 & 2 & 3.05 & 2.44 & 27.18 & 27.94 & -0.76 \\
\hline 2 & 2 & 3.05 & 2.44 & 30.73 & 30.73 & 0 \\
\hline 13 & 2 & 3.05 & 2.44 & 35.81 & 36.07 & -0.25 \\
\hline 19 & 2 & 3.05 & 2.44 & 36.83 & 36.58 & 0.25 \\
\hline 15 & 2 & 3.05 & 2.44 & 39.88 & 39.37 & 0.51 \\
\hline 1 & 2 & 3.05 & 3.66 & 26.92 & 27.43 & -0.51 \\
\hline 2 & 2 & 3.05 & 3.66 & 29.21 & 29.72 & -0.51 \\
\hline 19 & 2 & 3.05 & 3.66 & 36.07 & 36.32 & -0.25 \\
\hline 13 & 2 & 3.05 & 3.66 & 34.29 & 36.32 & -2.03 \\
\hline 15 & 2 & 3.05 & 3.66 & 37.85 & 39.12 & -1.27 \\
\hline 1 & 2 & 3.05 & 4.88 & 25.4 & 26.16 & -0.76 \\
\hline 2 & 2 & 3.05 & 4.88 & 27.69 & 28.7 & -1.02 \\
\hline 19 & 2 & 3.05 & 4.88 & 34.54 & 34.8 & -0.25 \\
\hline 13 & 2 & 3.05 & 4.88 & 33.78 & 36.07 & -2.29 \\
\hline 15 & 2 & 3.05 & 4.88 & 36.07 & 36.32 & -0.25 \\
\hline 1 & 2 & 3.05 & 6.1 & 24.64 & 25.4 & -0.76 \\
\hline 2 & 2 & 3.05 & 6.1 & 27.69 & 28.7 & -1.02 \\
\hline 13 & 2 & 3.05 & 6.1 & 32.77 & 33.53 & -0.76 \\
\hline 19 & 2 & 3.05 & 6.1 & 44.96 & 35.31 & 9.65 \\
\hline 15 & 2 & 3.05 & 6.1 & 33.78 & 35.31 & -1.52 \\
\hline 1 & 2 & 3.05 & 7.32 & 24.89 & 25.4 & -0.51 \\
\hline 2 & 2 & 3.05 & 7.32 & 26.42 & 27.18 & -0.76 \\
\hline 13 & 2 & 3.05 & 7.32 & 30.99 & 32.51 & -1.52 \\
\hline 15 & 2 & 3.05 & 7.32 & 36.58 & 33.78 & 2.79 \\
\hline 19 & 2 & 3.05 & 7.32 & 34.8 & 36.32 & -1.52 \\
\hline 1 & 2 & 3.05 & 8.53 & 25.91 & 24.13 & 1.78 \\
\hline 19 & 2 & 3.05 & 8.53 & 27.94 & 27.43 & 0.51 \\
\hline 2 & 2 & 3.05 & 8.53 & 25.91 & 27.43 & -1.52 \\
\hline 13 & 2 & 3.05 & 8.53 & 28.96 & 30.99 & -2.03 \\
\hline
\end{tabular}




$\begin{array}{lllllll}15 & 2 & 3.05 & 8.53 & 37.34 & 33.27 & 4.06 \\ 1 & 2 & 3.05 & 9.75 & 22.86 & 23.88 & -1.02 \\ 2 & 2 & 3.05 & 9.75 & 24.89 & 24.89 & 0 \\ 19 & 2 & 3.05 & 9.75 & 27.18 & 26.16 & 1.02 \\ 13 & 2 & 3.05 & 9.75 & 29.46 & 30.48 & -1.02 \\ 15 & 2 & 3.05 & 9.75 & 35.31 & 31.75 & 3.56 \\ 1 & 2 & 3.05 & 10.97 & 20.32 & 22.1 & -1.78 \\ 2 & 2 & 3.05 & 10.97 & 22.35 & 23.37 & -1.02 \\ 15 & 2 & 3.05 & 10.97 & 35.56 & 28.96 & 6.6 \\ 13 & 2 & 3.05 & 10.97 & 27.18 & 30.99 & -3.81 \\ 2 & 2 & 3.05 & 12.19 & 20.57 & 19.3 & 1.27 \\ 13 & 2 & 3.05 & 12.19 & 23.62 & 26.92 & -3.3 \\ 15 & 2 & 3.05 & 12.19 & 37.59 & 31.24 & 6.35 \\ 15 & 2 & 3.05 & 13.41 & 37.08 & 23.37 & 13.72 \\ 15 & 2 & 3.05 & 14.63 & 36.58 & 20.83 & 15.75 \\ 1 & 2 & 6.1 & 1.37 & 29.46 & 29.97 & -0.51 \\ 2 & 2 & 6.1 & 1.37 & 33.53 & 33.27 & 0.25 \\ 19 & 2 & 6.1 & 1.37 & 40.89 & 38.35 & 2.54 \\ 13 & 2 & 6.1 & 1.37 & 39.37 & 38.35 & 1.02 \\ 15 & 2 & 6.1 & 1.37 & 44.7 & 44.7 & 0 \\ 1 & 2 & 6.1 & 2.44 & 28.19 & 27.94 & 0.25 \\ 2 & 2 & 6.1 & 2.44 & 31.5 & 30.73 & 0.76 \\ 13 & 2 & 6.1 & 2.44 & 36.58 & 36.07 & 0.51 \\ 19 & 2 & 6.1 & 2.44 & 38.1 & 36.58 & 1.52 \\ 15 & 2 & 6.1 & 2.44 & 39.88 & 39.37 & 0.51 \\ 1 & 2 & 6.1 & 3.66 & 26.42 & 27.43 & -1.02 \\ 2 & 2 & 6.1 & 3.66 & 28.7 & 29.72 & -1.02 \\ 19 & 2 & 6.1 & 3.66 & 36.58 & 36.32 & 0.25 \\ 13 & 2 & 6.1 & 3.66 & 35.05 & 36.32 & -1.27 \\ 15 & 2 & 6.1 & 3.66 & 38.61 & 39.12 & -0.51 \\ 1 & 2 & 6.1 & 4.88 & 25.4 & 26.16 & -0.76 \\ 2 & 2 & 6.1 & 4.88 & 27.94 & 28.7 & -0.76 \\ 19 & 2 & 6.1 & 4.88 & 35.05 & 34.8 & 0.25 \\ 13 & 2 & 6.1 & 4.88 & 34.29 & 36.07 & -1.78 \\ 15 & 2 & 6.1 & 4.88 & 37.59 & 36.32 & 1.27 \\ 1 & 2 & 6.1 & 6.1 & 24.89 & 25.4 & -0.51 \\ 2 & 2 & 6.1 & 6.1 & 28.7 & 28.7 & 0 \\ 13 & 2 & 6.1 & 6.1 & 32 & 33.53 & -1.52 \\ 15 & 2 & 6.1 & 6.1 & 35.56 & 35.31 & 0.25 \\ 19 & 2 & 6.1 & 6.1 & 34.8 & 35.31 & -0.51 \\ 1 & 2 & 6.1 & 7.32 & 25.15 & 25.4 & -0.25 \\ 2 & 2 & 6.1 & 7.32 & 27.18 & 27.18 & 0 \\ 13 & 2 & 6.1 & 7.32 & 30.73 & 32.51 & -1.78 \\ 15 & 2 & 6.1 & 7.32 & 34.04 & 33.78 & 0.25 \\ 19 & 2 & 6.1 & 7.32 & 38.86 & 36.32 & 2.54 \\ 1 & 2 & 6.1 & 8.53 & 24.13 & 24.13 & 0 \\ 19 & 2 & 6.1 & 8.53 & 28.7 & 27.43 & 1.27 \\ 2 & 2 & 6.1 & 8.53 & 26.42 & 27.43 & -1.02 \\ 13 & 2 & 6.1 & 8.53 & 28.45 & 30.99 & -2.54 \\ 15 & 2 & 6.1 & 8.53 & 33.78 & 33.27 & 0.51 \\ 1 & 2 & 6.1 & 9.75 & 22.86 & 23.88 & -1.02 \\ 2 & 2 & 6.1 & 9.75 & 26.42 & 24.89 & 1.52 \\ 19 & 2 & 6.1 & 9.75 & 25.15 & 26.16 & -1.02 \\ 13 & 2 & 6.1 & 9.75 & 29.21 & 30.48 & -1.27\end{array}$




$\begin{array}{lllllll}15 & 2 & 6.1 & 9.75 & 28.7 & 31.75 & -3.05 \\ 1 & 2 & 6.1 & 10.97 & 21.08 & 22.1 & -1.02 \\ 2 & 2 & 6.1 & 10.97 & 24.89 & 23.37 & 1.52 \\ 19 & 2 & 6.1 & 10.97 & 23.62 & 23.37 & 0.25 \\ 15 & 2 & 6.1 & 10.97 & 27.69 & 28.96 & -1.27 \\ 13 & 2 & 6.1 & 10.97 & 28.45 & 30.99 & -2.54 \\ 2 & 2 & 6.1 & 12.19 & 20.07 & 19.3 & 0.76 \\ 19 & 2 & 6.1 & 12.19 & 21.08 & 22.35 & -1.27 \\ 1 & 2 & 6.1 & 12.19 & 19.81 & 23.11 & -3.3 \\ 13 & 2 & 6.1 & 12.19 & 24.64 & 26.92 & -2.29 \\ 15 & 2 & 6.1 & 12.19 & 30.23 & 31.24 & -1.02 \\ 1 & 2 & 6.1 & 13.41 & 18.03 & 19.56 & -1.52 \\ 15 & 2 & 6.1 & 13.41 & 29.46 & 23.37 & 6.1 \\ 13 & 2 & 6.1 & 13.41 & 23.88 & 27.69 & -3.81 \\ 1 & 2 & 6.1 & 14.63 & 16.51 & 18.54 & -2.03 \\ 15 & 2 & 6.1 & 14.63 & 28.96 & 20.83 & 8.13 \\ 13 & 2 & 6.1 & 14.63 & 19.05 & 23.37 & -4.32 \\ 1 & 2 & 6.1 & 15.85 & 15.49 & 16.51 & -1.02 \\ 1 & 2 & 6.1 & 17.07 & 12.7 & 14.22 & -1.52 \\ 1 & 2 & 6.1 & 18.29 & 12.7 & 11.94 & 0.76 \\ 1 & 2 & 9.14 & 1.37 & 29.46 & 29.97 & -0.51 \\ 2 & 2 & 9.14 & 1.37 & 34.8 & 33.27 & 1.52 \\ 19 & 2 & 9.14 & 1.37 & 40.64 & 38.35 & 2.29 \\ 13 & 2 & 9.14 & 1.37 & 40.13 & 38.35 & 1.78 \\ 15 & 2 & 9.14 & 1.37 & 43.43 & 44.7 & -1.27 \\ 1 & 2 & 9.14 & 2.44 & 28.19 & 27.94 & 0.25 \\ 2 & 2 & 9.14 & 2.44 & 31.75 & 30.73 & 1.02 \\ 13 & 2 & 9.14 & 2.44 & 37.08 & 36.07 & 1.02 \\ 19 & 2 & 9.14 & 2.44 & 38.86 & 36.58 & 2.29 \\ 15 & 2 & 9.14 & 2.44 & 39.62 & 39.37 & 0.25 \\ 1 & 2 & 9.14 & 3.66 & 27.18 & 27.43 & -0.25 \\ 2 & 2 & 9.14 & 3.66 & 28.96 & 29.72 & -0.76 \\ 19 & 2 & 9.14 & 3.66 & 36.58 & 36.32 & 0.25 \\ 13 & 2 & 9.14 & 3.66 & 35.31 & 36.32 & -1.02 \\ 15 & 2 & 9.14 & 3.66 & 39.37 & 39.12 & 0.25 \\ 1 & 2 & 9.14 & 4.88 & 25.4 & 26.16 & -0.76 \\ 2 & 2 & 9.14 & 4.88 & 28.19 & 28.7 & -0.51 \\ 19 & 2 & 9.14 & 4.88 & 36.07 & 34.8 & 1.27 \\ 13 & 2 & 9.14 & 4.88 & 34.8 & 36.07 & -1.27 \\ 15 & 2 & 9.14 & 4.88 & 37.34 & 36.32 & 1.02 \\ 1 & 2 & 9.14 & 6.1 & 24.38 & 25.4 & -1.02 \\ 2 & 2 & 9.14 & 6.1 & 27.69 & 28.7 & -1.02 \\ 13 & 2 & 9.14 & 6.1 & 32.77 & 33.53 & -0.76 \\ 19 & 2 & 9.14 & 6.1 & 37.34 & 35.31 & 2.03 \\ 15 & 2 & 9.14 & 6.1 & 35.56 & 35.31 & 0.25 \\ 1 & 2 & 9.14 & 7.32 & 24.38 & 25.4 & -1.02 \\ 2 & 2 & 9.14 & 7.32 & 27.18 & 27.18 & 0 \\ 13 & 2 & 9.14 & 7.32 & 32 & 32.51 & -0.51 \\ 15 & 2 & 9.14 & 7.32 & 35.31 & 33.78 & 1.52 \\ 19 & 2 & 9.14 & 7.32 & 40.89 & 36.32 & 4.57 \\ 1 & 2 & 9.14 & 8.53 & 23.11 & 24.13 & -1.02 \\ 19 & 2 & 9.14 & 8.53 & 32.51 & 27.43 & 5.08 \\ 2 & 2 & 9.14 & 8.53 & 25.91 & 27.43 & -1.52 \\ 13 & 2 & 9.14 & 8.53 & 30.48 & 30.99 & -0.51\end{array}$




\begin{tabular}{|c|c|c|c|c|c|c|}
\hline 15 & 2 & 9.14 & 8.53 & 33.78 & 33.27 & 0.51 \\
\hline 1 & 2 & 9.14 & 9.75 & 22.61 & 23.88 & -1.27 \\
\hline 2 & 2 & 9.14 & 9.75 & 25.91 & 24.89 & 1.02 \\
\hline 19 & 2 & 9.14 & 9.75 & 27.18 & 26.16 & 1.02 \\
\hline 13 & 2 & 9.14 & 9.75 & 29.46 & 30.48 & -1.02 \\
\hline 15 & 2 & 9.14 & 9.75 & 30.73 & 31.75 & -1.02 \\
\hline 1 & 2 & 9.14 & 10.97 & 23.11 & 22.1 & 1.02 \\
\hline 19 & 2 & 9.14 & 10.97 & 26.42 & 23.37 & 3.05 \\
\hline 2 & 2 & 9.14 & 10.97 & 23.11 & 23.37 & -0.25 \\
\hline 15 & 2 & 9.14 & 10.97 & 27.69 & 28.96 & -1.27 \\
\hline 13 & 2 & 9.14 & 10.97 & 28.7 & 30.99 & -2.29 \\
\hline 2 & 2 & 9.14 & 12.19 & 21.08 & 19.3 & 1.78 \\
\hline 19 & 2 & 9.14 & 12.19 & 23.37 & 22.35 & 1.02 \\
\hline 1 & 2 & 9.14 & 12.19 & 20.07 & 23.11 & -3.05 \\
\hline 13 & 2 & 9.14 & 12.19 & 26.67 & 26.92 & -0.25 \\
\hline 15 & 2 & 9.14 & 12.19 & 26.42 & 31.24 & -4.83 \\
\hline 1 & 2 & 9.14 & 13.41 & 19.81 & 19.56 & 0.25 \\
\hline 19 & 2 & 9.14 & 13.41 & 19.3 & 20.83 & -1.52 \\
\hline 15 & 2 & 9.14 & 13.41 & 23.37 & 23.37 & 0 \\
\hline 13 & 2 & 9.14 & 13.41 & 25.15 & 27.69 & -2.54 \\
\hline 1 & 2 & 9.14 & 14.63 & 16.76 & 18.54 & -1.78 \\
\hline 13 & 2 & 9.14 & 14.63 & 23.62 & 23.37 & 0.25 \\
\hline 1 & 2 & 9.14 & 15.85 & 14.22 & 16.51 & -2.29 \\
\hline 13 & 2 & 9.14 & 15.85 & 21.08 & 23.62 & -2.54 \\
\hline 1 & 2 & 12.19 & 1.37 & 28.45 & 29.97 & -1.52 \\
\hline 2 & 2 & 12.19 & 1.37 & 35.56 & 33.27 & 2.29 \\
\hline 19 & 2 & 12.19 & 1.37 & 41.4 & 38.35 & 3.05 \\
\hline 13 & 2 & 12.19 & 1.37 & 38.35 & 38.35 & 0 \\
\hline 15 & 2 & 12.19 & 1.37 & 41.91 & 44.7 & -2.79 \\
\hline 1 & 2 & 12.19 & 2.44 & 27.18 & 27.94 & -0.76 \\
\hline 2 & 2 & 12.19 & 2.44 & 31.75 & 30.73 & 1.02 \\
\hline 13 & 2 & 12.19 & 2.44 & 36.07 & 36.07 & 0 \\
\hline 19 & 2 & 12.19 & 2.44 & 38.1 & 36.58 & 1.52 \\
\hline 15 & 2 & 12.19 & 2.44 & 39.88 & 39.37 & 0.51 \\
\hline 1 & 2 & 12.19 & 3.66 & 27.18 & 27.43 & -0.25 \\
\hline 2 & 2 & 12.19 & 3.66 & 29.46 & 29.72 & -0.25 \\
\hline 19 & 2 & 12.19 & 3.66 & 36.07 & 36.32 & -0.25 \\
\hline 13 & 2 & 12.19 & 3.66 & 34.54 & 36.32 & -1.78 \\
\hline 15 & 2 & 12.19 & 3.66 & 39.12 & 39.12 & 0 \\
\hline 1 & 2 & 12.19 & 4.88 & 26.42 & 26.16 & 0.25 \\
\hline 2 & 2 & 12.19 & 4.88 & 27.18 & 28.7 & -1.52 \\
\hline 19 & 2 & 12.19 & 4.88 & 36.07 & 34.8 & 1.27 \\
\hline 13 & 2 & 12.19 & 4.88 & 33.27 & 36.07 & -2.79 \\
\hline 15 & 2 & 12.19 & 4.88 & 37.34 & 36.32 & 1.02 \\
\hline 1 & 2 & 12.19 & 6.1 & 26.16 & 25.4 & 0.76 \\
\hline 2 & 2 & 12.19 & 6.1 & 26.92 & 28.7 & -1.78 \\
\hline 13 & 2 & 12.19 & 6.1 & 32.77 & 33.53 & -0.76 \\
\hline 15 & 2 & 12.19 & 6.1 & 35.56 & 35.31 & 0.25 \\
\hline 19 & 2 & 12.19 & 6.1 & 35.05 & 35.31 & -0.25 \\
\hline 1 & 2 & 12.19 & 7.32 & 23.37 & 25.4 & -2.03 \\
\hline 2 & 2 & 12.19 & 7.32 & 25.4 & 27.18 & -1.78 \\
\hline 13 & 2 & 12.19 & 7.32 & 31.75 & 32.51 & -0.76 \\
\hline 15 & 2 & 12.19 & 7.32 & 34.54 & 33.78 & 0.76 \\
\hline 19 & 2 & 12.19 & 7.32 & 36.07 & 36.32 & -0.25 \\
\hline
\end{tabular}




\begin{tabular}{|c|c|c|c|c|c|c|}
\hline 1 & 2 & 12.19 & 8.53 & 23.37 & 24.13 & -0.76 \\
\hline 19 & 2 & 12.19 & 8.53 & 34.8 & 27.43 & 7.37 \\
\hline 2 & 2 & 12.19 & 8.53 & 27.43 & 27.43 & 0 \\
\hline 13 & 2 & 12.19 & 8.53 & 30.48 & 30.99 & -0.51 \\
\hline 15 & 2 & 12.19 & 8.53 & 33.53 & 33.27 & 0.25 \\
\hline 1 & 2 & 12.19 & 9.75 & 23.37 & 23.88 & -0.51 \\
\hline 2 & 2 & 12.19 & 9.75 & 26.16 & 24.89 & 1.27 \\
\hline 19 & 2 & 12.19 & 9.75 & 27.43 & 26.16 & 1.27 \\
\hline 13 & 2 & 12.19 & 9.75 & 28.96 & 30.48 & -1.52 \\
\hline 15 & 2 & 12.19 & 9.75 & 30.73 & 31.75 & -1.02 \\
\hline 1 & 2 & 12.19 & 10.97 & 22.86 & 22.1 & 0.76 \\
\hline 19 & 2 & 12.19 & 10.97 & 24.13 & 23.37 & 0.76 \\
\hline 2 & 2 & 12.19 & 10.97 & 23.62 & 23.37 & 0.25 \\
\hline 15 & 2 & 12.19 & 10.97 & 28.19 & 28.96 & -0.76 \\
\hline 13 & 2 & 12.19 & 10.97 & 28.45 & 30.99 & -2.54 \\
\hline 2 & 2 & 12.19 & 12.19 & 20.07 & 19.3 & 0.76 \\
\hline 19 & 2 & 12.19 & 12.19 & 23.62 & 22.35 & 1.27 \\
\hline 1 & 2 & 12.19 & 12.19 & 21.59 & 23.11 & -1.52 \\
\hline 13 & 2 & 12.19 & 12.19 & 26.42 & 26.92 & -0.51 \\
\hline 15 & 2 & 12.19 & 12.19 & 26.67 & 31.24 & -4.57 \\
\hline 1 & 2 & 12.19 & 13.41 & 20.07 & 19.56 & 0.51 \\
\hline 19 & 2 & 12.19 & 13.41 & 20.32 & 20.83 & -0.51 \\
\hline 15 & 2 & 12.19 & 13.41 & 23.88 & 23.37 & 0.51 \\
\hline 13 & 2 & 12.19 & 13.41 & 24.13 & 27.69 & -3.56 \\
\hline 1 & 2 & 12.19 & 14.63 & 17.53 & 18.54 & -1.02 \\
\hline 13 & 2 & 12.19 & 14.63 & 23.62 & 23.37 & 0.25 \\
\hline 13 & 2 & 12.19 & 15.85 & 21.59 & 23.62 & -2.03 \\
\hline 1 & 2 & 15.24 & 1.37 & 28.7 & 29.97 & -1.27 \\
\hline 2 & 2 & 15.24 & 1.37 & 34.04 & 33.27 & 0.76 \\
\hline 19 & 2 & 15.24 & 1.37 & 40.13 & 38.35 & 1.78 \\
\hline 13 & 2 & 15.24 & 1.37 & 38.35 & 38.35 & 0 \\
\hline 15 & 2 & 15.24 & 1.37 & 42.16 & 44.7 & -2.54 \\
\hline 1 & 2 & 15.24 & 2.44 & 27.18 & 27.94 & -0.76 \\
\hline 2 & 2 & 15.24 & 2.44 & 32.26 & 30.73 & 1.52 \\
\hline 13 & 2 & 15.24 & 2.44 & 36.58 & 36.07 & 0.51 \\
\hline 19 & 2 & 15.24 & 2.44 & 38.1 & 36.58 & 1.52 \\
\hline 1 & 2 & 15.24 & 3.66 & 26.67 & 27.43 & -0.76 \\
\hline 2 & 2 & 15.24 & 3.66 & 28.96 & 29.72 & -0.76 \\
\hline 19 & 2 & 15.24 & 3.66 & 35.81 & 36.32 & -0.51 \\
\hline 13 & 2 & 15.24 & 3.66 & 35.81 & 36.32 & -0.51 \\
\hline 15 & 2 & 15.24 & 3.66 & 40.13 & 39.12 & 1.02 \\
\hline 1 & 2 & 15.24 & 4.88 & 25.65 & 26.16 & -0.51 \\
\hline 2 & 2 & 15.24 & 4.88 & 27.94 & 28.7 & -0.76 \\
\hline 19 & 2 & 15.24 & 4.88 & 35.81 & 34.8 & 1.02 \\
\hline 13 & 2 & 15.24 & 4.88 & 34.29 & 36.07 & -1.78 \\
\hline 15 & 2 & 15.24 & 4.88 & 37.08 & 36.32 & 0.76 \\
\hline 1 & 2 & 15.24 & 6.1 & 23.88 & 25.4 & -1.52 \\
\hline 2 & 2 & 15.24 & 6.1 & 27.43 & 28.7 & -1.27 \\
\hline 13 & 2 & 15.24 & 6.1 & 32.51 & 33.53 & -1.02 \\
\hline 19 & 2 & 15.24 & 6.1 & 36.83 & 35.31 & 1.52 \\
\hline 15 & 2 & 15.24 & 6.1 & 35.56 & 35.31 & 0.25 \\
\hline 1 & 2 & 15.24 & 7.32 & 23.11 & 25.4 & -2.29 \\
\hline 2 & 2 & 15.24 & 7.32 & 24.89 & 27.18 & -2.29 \\
\hline 13 & 2 & 15.24 & 7.32 & 31.75 & 32.51 & -0.76 \\
\hline
\end{tabular}




\begin{tabular}{|c|c|c|c|c|c|c|}
\hline 15 & 2 & 15.24 & 7.32 & 35.31 & 33.78 & 1.52 \\
\hline 19 & 2 & 15.24 & 7.32 & 34.29 & 36.32 & -2.03 \\
\hline 1 & 2 & 15.24 & 8.53 & 23.88 & 24.13 & -0.25 \\
\hline 19 & 2 & 15.24 & 8.53 & 35.56 & 27.43 & 8.13 \\
\hline 2 & 2 & 15.24 & 8.53 & 24.89 & 27.43 & -2.54 \\
\hline 13 & 2 & 15.24 & 8.53 & 30.23 & 30.99 & -0.76 \\
\hline 15 & 2 & 15.24 & 8.53 & 33.02 & 33.27 & -0.25 \\
\hline 1 & 2 & 15.24 & 9.75 & 22.61 & 23.88 & -1.27 \\
\hline 2 & 2 & 15.24 & 9.75 & 23.37 & 24.89 & -1.52 \\
\hline 19 & 2 & 15.24 & 9.75 & 28.19 & 26.16 & 2.03 \\
\hline 13 & 2 & 15.24 & 9.75 & 30.23 & 30.48 & -0.25 \\
\hline 15 & 2 & 15.24 & 9.75 & 35.05 & 31.75 & 3.3 \\
\hline 1 & 2 & 15.24 & 10.97 & 21.84 & 22.1 & -0.25 \\
\hline 19 & 2 & 15.24 & 10.97 & 24.38 & 23.37 & 1.02 \\
\hline 2 & 2 & 15.24 & 10.97 & 20.83 & 23.37 & -2.54 \\
\hline 15 & 2 & 15.24 & 10.97 & 30.23 & 28.96 & 1.27 \\
\hline 13 & 2 & 15.24 & 10.97 & 29.46 & 30.99 & -1.52 \\
\hline 2 & 2 & 15.24 & 12.19 & 19.56 & 19.3 & 0.25 \\
\hline 19 & 2 & 15.24 & 12.19 & 23.37 & 22.35 & 1.02 \\
\hline 1 & 2 & 15.24 & 12.19 & 20.07 & 23.11 & -3.05 \\
\hline 13 & 2 & 15.24 & 12.19 & 25.65 & 26.92 & -1.27 \\
\hline 15 & 2 & 15.24 & 12.19 & 28.19 & 31.24 & -3.05 \\
\hline 19 & 2 & 15.24 & 13.41 & 21.08 & 20.83 & 0.25 \\
\hline 13 & 2 & 15.24 & 13.41 & 25.15 & 27.69 & -2.54 \\
\hline 1 & 2 & 15.24 & 14.63 & 15.75 & 18.54 & -2.79 \\
\hline 19 & 2 & 15.24 & 14.63 & 19.3 & 20.07 & -0.76 \\
\hline 13 & 2 & 15.24 & 14.63 & 23.62 & 23.37 & 0.25 \\
\hline 1 & 2 & 15.24 & 15.85 & 13.97 & 16.51 & -2.54 \\
\hline 13 & 2 & 15.24 & 15.85 & 20.07 & 23.62 & -3.56 \\
\hline 7 & 3 & 3.05 & 1.37 & 46.99 & 47.24 & -0.25 \\
\hline 17 & 3 & 3.05 & 1.37 & 51.82 & 50.8 & 1.02 \\
\hline 12 & 3 & 3.05 & 1.37 & 53.09 & 53.34 & -0.25 \\
\hline 20 & 3 & 3.05 & 1.37 & 58.67 & 56.64 & 2.03 \\
\hline 6 & 3 & 3.05 & 2.44 & 38.86 & 41.91 & -3.05 \\
\hline 7 & 3 & 3.05 & 2.44 & 42.93 & 43.43 & -0.51 \\
\hline 17 & 3 & 3.05 & 2.44 & 46.74 & 49.28 & -2.54 \\
\hline 12 & 3 & 3.05 & 2.44 & 48.26 & 50.04 & -1.78 \\
\hline 20 & 3 & 3.05 & 2.44 & 51.56 & 51.05 & 0.51 \\
\hline 6 & 3 & 3.05 & 3.66 & 36.58 & 40.64 & -4.06 \\
\hline 7 & 3 & 3.05 & 3.66 & 38.1 & 40.89 & -2.79 \\
\hline 17 & 3 & 3.05 & 3.66 & 44.7 & 47.24 & -2.54 \\
\hline 12 & 3 & 3.05 & 3.66 & 45.97 & 47.5 & -1.52 \\
\hline 20 & 3 & 3.05 & 3.66 & 51.05 & 50.8 & 0.25 \\
\hline 7 & 3 & 3.05 & 4.88 & 36.32 & 38.1 & -1.78 \\
\hline 6 & 3 & 3.05 & 4.88 & 38.1 & 39.37 & -1.27 \\
\hline 12 & 3 & 3.05 & 4.88 & 44.2 & 45.72 & -1.52 \\
\hline 17 & 3 & 3.05 & 4.88 & 42.67 & 46.48 & -3.81 \\
\hline 20 & 3 & 3.05 & 4.88 & 48.77 & 49.78 & -1.02 \\
\hline 6 & 3 & 3.05 & 6.1 & 29.21 & 27.43 & 1.78 \\
\hline 7 & 3 & 3.05 & 6.1 & 44.45 & 35.56 & 8.89 \\
\hline 17 & 3 & 3.05 & 6.1 & 43.43 & 44.96 & -1.52 \\
\hline 20 & 3 & 3.05 & 6.1 & 51.05 & 48.77 & 2.29 \\
\hline 6 & 3 & 3.05 & 7.32 & 26.67 & 26.67 & 0 \\
\hline 7 & 3 & 3.05 & 7.32 & 46.99 & 34.54 & 12.45 \\
\hline
\end{tabular}




$\begin{array}{lllllll}17 & 3 & 3.05 & 7.32 & 40.64 & 45.72 & -5.08 \\ 20 & 3 & 3.05 & 7.32 & 44.7 & 47.5 & -2.79 \\ 6 & 3 & 3.05 & 8.53 & 26.42 & 25.91 & 0.51 \\ 7 & 3 & 3.05 & 8.53 & 39.88 & 33.53 & 6.35 \\ 17 & 3 & 3.05 & 8.53 & 36.58 & 43.69 & -7.11 \\ 20 & 3 & 3.05 & 8.53 & 42.16 & 45.97 & -3.81 \\ 6 & 3 & 3.05 & 9.75 & 24.64 & 26.16 & -1.52 \\ 7 & 3 & 3.05 & 9.75 & 32.77 & 32.77 & 0 \\ 17 & 3 & 3.05 & 9.75 & 33.53 & 42.16 & -8.64 \\ 20 & 3 & 3.05 & 9.75 & 38.35 & 45.21 & -6.86 \\ 6 & 3 & 3.05 & 10.97 & 22.61 & 24.38 & -1.78 \\ 7 & 3 & 3.05 & 10.97 & 31.75 & 33.27 & -1.52 \\ 20 & 3 & 3.05 & 10.97 & 39.62 & 38.86 & 0.76 \\ 6 & 3 & 3.05 & 12.19 & 21.34 & 18.03 & 3.3 \\ 7 & 3 & 3.05 & 12.19 & 27.43 & 31.24 & -3.81 \\ 20 & 3 & 3.05 & 12.19 & 35.56 & 35.81 & -0.25 \\ 7 & 3 & 3.05 & 13.41 & 27.18 & 21.08 & 6.1 \\ 20 & 3 & 3.05 & 13.41 & 31.5 & 34.54 & -3.05 \\ 7 & 3 & 3.05 & 14.63 & 27.18 & 20.57 & 6.6 \\ 7 & 3 & 3.05 & 15.85 & 24.13 & 17.27 & 6.86 \\ 7 & 3 & 3.05 & 17.07 & 25.4 & 16.26 & 9.14 \\ 7 & 3 & 6.1 & 1.37 & 47.24 & 47.24 & 0 \\ 17 & 3 & 6.1 & 1.37 & 52.32 & 50.8 & 1.52 \\ 12 & 3 & 6.1 & 1.37 & 53.34 & 53.34 & 0 \\ 20 & 3 & 6.1 & 1.37 & 57.4 & 56.64 & 0.76 \\ 6 & 3 & 6.1 & 2.44 & 39.88 & 41.91 & -2.03 \\ 7 & 3 & 6.1 & 2.44 & 43.69 & 43.43 & 0.25 \\ 17 & 3 & 6.1 & 2.44 & 48.77 & 49.28 & -0.51 \\ 12 & 3 & 6.1 & 2.44 & 50.04 & 50.04 & 0 \\ 20 & 3 & 6.1 & 2.44 & 53.59 & 51.05 & 2.54 \\ 6 & 3 & 6.1 & 3.66 & 37.85 & 40.64 & -2.79 \\ 7 & 3 & 6.1 & 3.66 & 39.88 & 40.89 & -1.02 \\ 17 & 3 & 6.1 & 3.66 & 46.48 & 47.24 & -0.76 \\ 12 & 3 & 6.1 & 3.66 & 46.99 & 47.5 & -0.51 \\ 20 & 3 & 6.1 & 3.66 & 52.32 & 50.8 & 1.52 \\ 7 & 3 & 6.1 & 4.88 & 37.85 & 38.1 & -0.25 \\ 6 & 3 & 6.1 & 4.88 & 36.83 & 39.37 & -2.54 \\ 12 & 3 & 6.1 & 4.88 & 46.23 & 45.72 & 0.51 \\ 17 & 3 & 6.1 & 4.88 & 45.97 & 46.48 & -0.51 \\ 20 & 3 & 6.1 & 4.88 & 50.29 & 49.78 & 0.51 \\ 6 & 3 & 6.1 & 6.1 & 32.77 & 27.43 & 5.33 \\ 7 & 3 & 6.1 & 6.1 & 38.86 & 35.56 & 3.3 \\ 17 & 3 & 6.1 & 6.1 & 44.96 & 44.96 & 0 \\ 12 & 3 & 6.1 & 6.1 & 46.23 & 46.74 & -0.51 \\ 20 & 3 & 6.1 & 6.1 & 48.26 & 48.77 & -0.51 \\ 6 & 3 & 6.1 & 7.32 & 30.99 & 26.67 & 4.32 \\ 7 & 3 & 6.1 & 7.32 & 35.81 & 34.54 & 1.27 \\ 17 & 3 & 6.1 & 7.32 & 44.2 & 45.72 & -1.52 \\ 12 & 3 & 6.1 & 7.32 & 43.43 & 45.72 & -2.29 \\ 20 & 3 & 6.1 & 7.32 & 49.02 & 47.5 & 1.52 \\ 6 & 3 & 6.1 & 8.53 & 29.72 & 25.91 & 3.81 \\ 7 & 3 & 6.1 & 8.53 & 34.04 & 33.53 & 0.51 \\ 17 & 3 & 6.1 & 8.53 & 42.67 & 43.69 & -1.02 \\ 12 & 3 & 6.1 & 8.53 & 43.43 & 44.7 & -1.27\end{array}$




$\begin{array}{lllllll}20 & 3 & 6.1 & 8.53 & 47.75 & 45.97 & 1.78 \\ 6 & 3 & 6.1 & 9.75 & 28.7 & 26.16 & 2.54 \\ 7 & 3 & 6.1 & 9.75 & 33.02 & 32.77 & 0.25 \\ 17 & 3 & 6.1 & 9.75 & 42.42 & 42.16 & 0.25 \\ 12 & 3 & 6.1 & 9.75 & 41.15 & 43.18 & -2.03 \\ 20 & 3 & 6.1 & 9.75 & 43.94 & 45.21 & -1.27 \\ 6 & 3 & 6.1 & 10.97 & 27.18 & 24.38 & 2.79 \\ 7 & 3 & 6.1 & 10.97 & 31.75 & 33.27 & -1.52 \\ 20 & 3 & 6.1 & 10.97 & 44.96 & 38.86 & 6.1 \\ 17 & 3 & 6.1 & 10.97 & 37.59 & 41.91 & -4.32 \\ 12 & 3 & 6.1 & 10.97 & 39.62 & 43.43 & -3.81 \\ 6 & 3 & 6.1 & 12.19 & 23.11 & 18.03 & 5.08 \\ 7 & 3 & 6.1 & 12.19 & 28.45 & 31.24 & -2.79 \\ 20 & 3 & 6.1 & 12.19 & 36.07 & 35.81 & 0.25 \\ 17 & 3 & 6.1 & 12.19 & 36.32 & 41.4 & -5.08 \\ 12 & 3 & 6.1 & 12.19 & 37.85 & 42.42 & -4.57 \\ 7 & 3 & 6.1 & 13.41 & 26.67 & 21.08 & 5.59 \\ 20 & 3 & 6.1 & 13.41 & 36.07 & 34.54 & 1.52 \\ 12 & 3 & 6.1 & 13.41 & 35.56 & 40.89 & -5.33 \\ 7 & 3 & 6.1 & 14.63 & 22.1 & 20.57 & 1.52 \\ 17 & 3 & 6.1 & 14.63 & 36.32 & 32 & 4.32 \\ 20 & 3 & 6.1 & 14.63 & 34.29 & 34.54 & -0.25 \\ 7 & 3 & 6.1 & 15.85 & 21.08 & 17.27 & 3.81 \\ 17 & 3 & 6.1 & 15.85 & 30.23 & 31.24 & -1.02 \\ 7 & 3 & 6.1 & 17.07 & 18.29 & 16.26 & 2.03 \\ 7 & 3 & 9.14 & 1.37 & 47.24 & 47.24 & 0 \\ 17 & 3 & 9.14 & 1.37 & 51.31 & 50.8 & 0.51 \\ 12 & 3 & 9.14 & 1.37 & 52.58 & 53.34 & -0.76 \\ 20 & 3 & 9.14 & 1.37 & 58.17 & 56.64 & 1.52 \\ 6 & 3 & 9.14 & 2.44 & 42.16 & 41.91 & 0.25 \\ 7 & 3 & 9.14 & 2.44 & 43.94 & 43.43 & 0.51 \\ 17 & 3 & 9.14 & 2.44 & 49.02 & 49.28 & -0.25 \\ 12 & 3 & 9.14 & 2.44 & 49.28 & 50.04 & -0.76 \\ 20 & 3 & 9.14 & 2.44 & 53.59 & 51.05 & 2.54 \\ 6 & 3 & 9.14 & 3.66 & 38.61 & 40.64 & -2.03 \\ 7 & 3 & 9.14 & 3.66 & 40.39 & 40.89 & -0.51 \\ 17 & 3 & 9.14 & 3.66 & 47.5 & 47.24 & 0.25 \\ 12 & 3 & 9.14 & 3.66 & 47.24 & 47.5 & -0.25 \\ 20 & 3 & 9.14 & 3.66 & 52.58 & 50.8 & 1.78 \\ 7 & 3 & 9.14 & 4.88 & 36.83 & 38.1 & -1.27 \\ 6 & 3 & 9.14 & 4.88 & 39.12 & 39.37 & -0.25 \\ 12 & 3 & 9.14 & 4.88 & 45.97 & 45.72 & 0.25 \\ 17 & 3 & 9.14 & 4.88 & 46.48 & 46.48 & 0 \\ 20 & 3 & 9.14 & 4.88 & 49.02 & 49.78 & -0.76 \\ 6 & 3 & 9.14 & 6.1 & 29.21 & 27.43 & 1.78 \\ 7 & 3 & 9.14 & 6.1 & 34.8 & 35.56 & -0.76 \\ 17 & 3 & 9.14 & 6.1 & 44.7 & 44.96 & -0.25 \\ 12 & 3 & 9.14 & 6.1 & 45.97 & 46.74 & -0.76 \\ 20 & 3 & 9.14 & 6.1 & 47.75 & 48.77 & -1.02 \\ 6 & 3 & 9.14 & 7.32 & 29.72 & 26.67 & 3.05 \\ 7 & 3 & 9.14 & 7.32 & 35.31 & 34.54 & 0.76 \\ 17 & 3 & 9.14 & 7.32 & 44.2 & 45.72 & -1.52 \\ 12 & 3 & 9.14 & 7.32 & 44.2 & 45.72 & -1.52 \\ 20 & 3 & 9.14 & 7.32 & 47.75 & 47.5 & 0.25\end{array}$




$\begin{array}{lllllll}6 & 3 & 9.14 & 8.53 & 25.91 & 25.91 & 0 \\ 7 & 3 & 9.14 & 8.53 & 32 & 33.53 & -1.52 \\ 17 & 3 & 9.14 & 8.53 & 43.18 & 43.69 & -0.51 \\ 12 & 3 & 9.14 & 8.53 & 44.7 & 44.7 & 0 \\ 20 & 3 & 9.14 & 8.53 & 48.01 & 45.97 & 2.03 \\ 6 & 3 & 9.14 & 9.75 & 29.21 & 26.16 & 3.05 \\ 7 & 3 & 9.14 & 9.75 & 31.5 & 32.77 & -1.27 \\ 17 & 3 & 9.14 & 9.75 & 43.18 & 42.16 & 1.02 \\ 12 & 3 & 9.14 & 9.75 & 42.42 & 43.18 & -0.76 \\ 20 & 3 & 9.14 & 9.75 & 42.93 & 45.21 & -2.29 \\ 6 & 3 & 9.14 & 10.97 & 28.96 & 24.38 & 4.57 \\ 7 & 3 & 9.14 & 10.97 & 30.48 & 33.27 & -2.79 \\ 20 & 3 & 9.14 & 10.97 & 48.01 & 38.86 & 9.14 \\ 17 & 3 & 9.14 & 10.97 & 40.89 & 41.91 & -1.02 \\ 12 & 3 & 9.14 & 10.97 & 42.93 & 43.43 & -0.51 \\ 6 & 3 & 9.14 & 12.19 & 24.13 & 18.03 & 6.1 \\ 7 & 3 & 9.14 & 12.19 & 24.89 & 31.24 & -6.35 \\ 20 & 3 & 9.14 & 12.19 & 34.04 & 35.81 & -1.78 \\ 17 & 3 & 9.14 & 12.19 & 38.1 & 41.4 & -3.3 \\ 12 & 3 & 9.14 & 12.19 & 40.13 & 42.42 & -2.29 \\ 7 & 3 & 9.14 & 13.41 & 22.61 & 21.08 & 1.52 \\ 20 & 3 & 9.14 & 13.41 & 33.53 & 34.54 & -1.02 \\ 12 & 3 & 9.14 & 13.41 & 37.08 & 40.89 & -3.81 \\ 7 & 3 & 9.14 & 14.63 & 19.56 & 20.57 & -1.02 \\ 20 & 3 & 9.14 & 14.63 & 34.8 & 34.54 & 0.25 \\ 7 & 3 & 9.14 & 15.85 & 15.75 & 17.27 & -1.52 \\ 7 & 3 & 9.14 & 17.07 & 16.51 & 16.26 & 0.25 \\ 7 & 3 & 12.19 & 1.37 & 46.48 & 47.24 & -0.76 \\ 17 & 3 & 12.19 & 1.37 & 52.58 & 50.8 & 1.78 \\ 12 & 3 & 12.19 & 1.37 & 52.32 & 53.34 & -1.02 \\ 20 & 3 & 12.19 & 1.37 & 58.17 & 56.64 & 1.52 \\ 6 & 3 & 12.19 & 2.44 & 41.66 & 41.91 & -0.25 \\ 7 & 3 & 12.19 & 2.44 & 43.94 & 43.43 & 0.51 \\ 17 & 3 & 12.19 & 2.44 & 49.02 & 49.28 & -0.25 \\ 12 & 3 & 12.19 & 2.44 & 50.04 & 50.04 & 0 \\ 20 & 3 & 12.19 & 2.44 & 53.85 & 51.05 & 2.79 \\ 6 & 3 & 12.19 & 3.66 & 40.64 & 40.64 & 0 \\ 7 & 3 & 12.19 & 3.66 & 41.4 & 40.89 & 0.51 \\ 17 & 3 & 12.19 & 3.66 & 47.5 & 47.24 & 0.25 \\ 12 & 3 & 12.19 & 3.66 & 47.5 & 47.5 & 0 \\ 20 & 3 & 12.19 & 3.66 & 52.83 & 50.8 & 2.03 \\ 7 & 3 & 12.19 & 4.88 & 36.83 & 38.1 & -1.27 \\ 6 & 3 & 12.19 & 4.88 & 41.66 & 39.37 & 2.29 \\ 12 & 3 & 12.19 & 4.88 & 46.23 & 45.72 & 0.51 \\ 17 & 3 & 12.19 & 4.88 & 46.23 & 46.48 & -0.25 \\ 20 & 3 & 12.19 & 4.88 & 49.78 & 49.78 & 0 \\ 6 & 3 & 12.19 & 6.1 & 26.92 & 27.43 & -0.51 \\ 7 & 3 & 12.19 & 6.1 & 35.05 & 35.56 & -0.51 \\ 17 & 3 & 12.19 & 6.1 & 44.2 & 44.96 & -0.76 \\ 12 & 3 & 12.19 & 6.1 & 46.74 & 46.74 & 0 \\ 20 & 3 & 12.19 & 6.1 & 48.26 & 48.77 & -0.51 \\ 6 & 3 & 12.19 & 7.32 & 26.67 & 26.67 & 0 \\ 7 & 3 & 12.19 & 7.32 & 33.53 & 34.54 & -1.02 \\ 17 & 3 & 12.19 & 7.32 & 44.45 & 45.72 & -1.27\end{array}$




\begin{tabular}{|c|c|c|c|c|c|c|}
\hline 12 & 3 & 12.19 & 7.32 & 44.2 & 45.72 & -1.52 \\
\hline 20 & 3 & 12.19 & 7.32 & 46.99 & 47.5 & -0.51 \\
\hline 6 & 3 & 12.19 & 8.53 & 26.67 & 25.91 & 0.76 \\
\hline 7 & 3 & 12.19 & 8.53 & 34.29 & 33.53 & 0.76 \\
\hline 17 & 3 & 12.19 & 8.53 & 44.2 & 43.69 & 0.51 \\
\hline 12 & 3 & 12.19 & 8.53 & 44.7 & 44.7 & 0 \\
\hline 20 & 3 & 12.19 & 8.53 & 48.51 & 45.97 & 2.54 \\
\hline 6 & 3 & 12.19 & 9.75 & 24.64 & 26.16 & -1.52 \\
\hline 7 & 3 & 12.19 & 9.75 & 32 & 32.77 & -0.76 \\
\hline 17 & 3 & 12.19 & 9.75 & 42.93 & 42.16 & 0.76 \\
\hline 12 & 3 & 12.19 & 9.75 & 43.69 & 43.18 & 0.51 \\
\hline 20 & 3 & 12.19 & 9.75 & 49.53 & 45.21 & 4.32 \\
\hline 6 & 3 & 12.19 & 10.97 & 24.38 & 24.38 & 0 \\
\hline 7 & 3 & 12.19 & 10.97 & 31.5 & 33.27 & -1.78 \\
\hline 20 & 3 & 12.19 & 10.97 & 47.5 & 38.86 & 8.64 \\
\hline 17 & 3 & 12.19 & 10.97 & 41.4 & 41.91 & -0.51 \\
\hline 12 & 3 & 12.19 & 10.97 & 42.16 & 43.43 & -1.27 \\
\hline 6 & 3 & 12.19 & 12.19 & 20.32 & 18.03 & 2.29 \\
\hline 7 & 3 & 12.19 & 12.19 & 30.99 & 31.24 & -0.25 \\
\hline 20 & 3 & 12.19 & 12.19 & 36.32 & 35.81 & 0.51 \\
\hline 12 & 3 & 12.19 & 12.19 & 40.89 & 42.42 & -1.52 \\
\hline 7 & 3 & 12.19 & 13.41 & 23.88 & 21.08 & 2.79 \\
\hline 20 & 3 & 12.19 & 13.41 & 33.02 & 34.54 & -1.52 \\
\hline 12 & 3 & 12.19 & 13.41 & 38.1 & 40.89 & -2.79 \\
\hline 7 & 3 & 12.19 & 14.63 & 19.3 & 20.57 & -1.27 \\
\hline 17 & 3 & 12.19 & 14.63 & 35.31 & 32 & 3.3 \\
\hline 20 & 3 & 12.19 & 14.63 & 32.77 & 34.54 & -1.78 \\
\hline 12 & 3 & 12.19 & 14.63 & 36.83 & 38.1 & -1.27 \\
\hline 7 & 3 & 12.19 & 15.85 & 18.03 & 17.27 & 0.76 \\
\hline 17 & 3 & 12.19 & 15.85 & 30.73 & 31.24 & -0.51 \\
\hline 7 & 3 & 12.19 & 17.07 & 13.72 & 16.26 & -2.54 \\
\hline 7 & 3 & 15.24 & 1.37 & 45.72 & 47.24 & -1.52 \\
\hline 17 & 3 & 15.24 & 1.37 & 52.32 & 50.8 & 1.52 \\
\hline 12 & 3 & 15.24 & 1.37 & 52.07 & 53.34 & -1.27 \\
\hline 20 & 3 & 15.24 & 1.37 & 57.4 & 56.64 & 0.76 \\
\hline 6 & 3 & 15.24 & 2.44 & 45.21 & 41.91 & 3.3 \\
\hline 7 & 3 & 15.24 & 2.44 & 42.93 & 43.43 & -0.51 \\
\hline 17 & 3 & 15.24 & 2.44 & 50.04 & 49.28 & 0.76 \\
\hline 12 & 3 & 15.24 & 2.44 & 49.53 & 50.04 & -0.51 \\
\hline 20 & 3 & 15.24 & 2.44 & 53.59 & 51.05 & 2.54 \\
\hline 6 & 3 & 15.24 & 3.66 & 42.42 & 40.64 & 1.78 \\
\hline 7 & 3 & 15.24 & 3.66 & 40.13 & 40.89 & -0.76 \\
\hline 17 & 3 & 15.24 & 3.66 & 48.26 & 47.24 & 1.02 \\
\hline 12 & 3 & 15.24 & 3.66 & 46.23 & 47.5 & -1.27 \\
\hline 20 & 3 & 15.24 & 3.66 & 52.83 & 50.8 & 2.03 \\
\hline 7 & 3 & 15.24 & 4.88 & 37.34 & 38.1 & -0.76 \\
\hline 6 & 3 & 15.24 & 4.88 & 43.18 & 39.37 & 3.81 \\
\hline 12 & 3 & 15.24 & 4.88 & 45.97 & 45.72 & 0.25 \\
\hline 17 & 3 & 15.24 & 4.88 & 46.48 & 46.48 & 0 \\
\hline 20 & 3 & 15.24 & 4.88 & 51.05 & 49.78 & 1.27 \\
\hline 6 & 3 & 15.24 & 6.1 & 28.96 & 27.43 & 1.52 \\
\hline 7 & 3 & 15.24 & 6.1 & 34.29 & 35.56 & -1.27 \\
\hline 17 & 3 & 15.24 & 6.1 & 44.7 & 44.96 & -0.25 \\
\hline 12 & 3 & 15.24 & 6.1 & 46.23 & 46.74 & -0.51 \\
\hline
\end{tabular}




\begin{tabular}{|c|c|c|c|c|c|c|}
\hline 20 & 3 & 15.24 & 6.1 & 49.02 & 48.77 & 0.25 \\
\hline 6 & 3 & 15.24 & 7.32 & 27.18 & 26.67 & 0.51 \\
\hline 7 & 3 & 15.24 & 7.32 & 32.77 & 34.54 & -1.78 \\
\hline 12 & 3 & 15.24 & 7.32 & 44.45 & 45.72 & -1.27 \\
\hline 17 & 3 & 15.24 & 7.32 & 44.2 & 45.72 & -1.52 \\
\hline 20 & 3 & 15.24 & 7.32 & 46.99 & 47.5 & -0.51 \\
\hline 6 & 3 & 15.24 & 8.53 & 25.4 & 25.91 & -0.51 \\
\hline 7 & 3 & 15.24 & 8.53 & 30.99 & 33.53 & -2.54 \\
\hline 17 & 3 & 15.24 & 8.53 & 44.2 & 43.69 & 0.51 \\
\hline 12 & 3 & 15.24 & 8.53 & 43.69 & 44.7 & -1.02 \\
\hline 20 & 3 & 15.24 & 8.53 & 48.01 & 45.97 & 2.03 \\
\hline 6 & 3 & 15.24 & 9.75 & 25.91 & 26.16 & -0.25 \\
\hline 7 & 3 & 15.24 & 9.75 & 30.99 & 32.77 & -1.78 \\
\hline 17 & 3 & 15.24 & 9.75 & 43.18 & 42.16 & 1.02 \\
\hline 12 & 3 & 15.24 & 9.75 & 41.91 & 43.18 & -1.27 \\
\hline 20 & 3 & 15.24 & 9.75 & 49.53 & 45.21 & 4.32 \\
\hline 6 & 3 & 15.24 & 10.97 & 23.11 & 24.38 & -1.27 \\
\hline 7 & 3 & 15.24 & 10.97 & 29.97 & 33.27 & -3.3 \\
\hline 20 & 3 & 15.24 & 10.97 & 48.77 & 38.86 & 9.91 \\
\hline 17 & 3 & 15.24 & 10.97 & 41.15 & 41.91 & -0.76 \\
\hline 12 & 3 & 15.24 & 10.97 & 42.42 & 43.43 & -1.02 \\
\hline 6 & 3 & 15.24 & 12.19 & 21.59 & 18.03 & 3.56 \\
\hline 7 & 3 & 15.24 & 12.19 & 28.96 & 31.24 & -2.29 \\
\hline 20 & 3 & 15.24 & 12.19 & 36.32 & 35.81 & 0.51 \\
\hline 12 & 3 & 15.24 & 12.19 & 41.4 & 42.42 & -1.02 \\
\hline 7 & 3 & 15.24 & 13.41 & 21.59 & 21.08 & 0.51 \\
\hline 20 & 3 & 15.24 & 13.41 & 34.04 & 34.54 & -0.51 \\
\hline 12 & 3 & 15.24 & 13.41 & 39.37 & 40.89 & -1.52 \\
\hline 7 & 3 & 15.24 & 14.63 & 19.3 & 20.57 & -1.27 \\
\hline 17 & 3 & 15.24 & 14.63 & 33.53 & 32 & 1.52 \\
\hline 20 & 3 & 15.24 & 14.63 & 34.54 & 34.54 & 0 \\
\hline 12 & 3 & 15.24 & 14.63 & 37.34 & 38.1 & -0.76 \\
\hline 7 & 3 & 15.24 & 15.85 & 14.99 & 17.27 & -2.29 \\
\hline 17 & 3 & 15.24 & 15.85 & 28.96 & 31.24 & -2.29 \\
\hline 7 & 3 & 15.24 & 17.07 & 13.46 & 16.26 & -2.79 \\
\hline 4 & 4 & 3.05 & 1.37 & 55.88 & 55.37 & 0.51 \\
\hline 16 & 4 & 3.05 & 1.37 & 61.72 & 60.45 & 1.27 \\
\hline 5 & 4 & 3.05 & 1.37 & 62.23 & 61.21 & 1.02 \\
\hline 14 & 4 & 3.05 & 1.37 & 69.09 & 66.29 & 2.79 \\
\hline 4 & 4 & 3.05 & 2.44 & 50.8 & 52.83 & -2.03 \\
\hline 16 & 4 & 3.05 & 2.44 & 56.9 & 56.13 & 0.76 \\
\hline 5 & 4 & 3.05 & 2.44 & 57.66 & 58.67 & -1.02 \\
\hline 14 & 4 & 3.05 & 2.44 & 62.23 & 63.75 & -1.52 \\
\hline 4 & 4 & 3.05 & 3.66 & 49.78 & 51.82 & -2.03 \\
\hline 16 & 4 & 3.05 & 3.66 & 55.12 & 54.86 & 0.25 \\
\hline 5 & 4 & 3.05 & 3.66 & 54.86 & 57.91 & -3.05 \\
\hline 14 & 4 & 3.05 & 3.66 & 60.45 & 62.48 & -2.03 \\
\hline 4 & 4 & 3.05 & 4.88 & 48.26 & 51.05 & -2.79 \\
\hline 16 & 4 & 3.05 & 4.88 & 53.85 & 53.34 & 0.51 \\
\hline 5 & 4 & 3.05 & 4.88 & 53.09 & 56.13 & -3.05 \\
\hline 14 & 4 & 3.05 & 4.88 & 58.42 & 60.96 & -2.54 \\
\hline 16 & 4 & 3.05 & 6.1 & 52.58 & 52.58 & 0 \\
\hline 4 & 4 & 3.05 & 6.1 & 47.24 & 52.58 & -5.33 \\
\hline 5 & 4 & 3.05 & 6.1 & 54.1 & 54.61 & -0.51 \\
\hline
\end{tabular}




$\begin{array}{lllllll}14 & 4 & 3.05 & 6.1 & 61.72 & 60.96 & 0.76 \\ 4 & 4 & 3.05 & 7.32 & 45.97 & 49.53 & -3.56 \\ 16 & 4 & 3.05 & 7.32 & 51.82 & 52.07 & -0.25 \\ 5 & 4 & 3.05 & 7.32 & 53.34 & 56.64 & -3.3 \\ 14 & 4 & 3.05 & 7.32 & 57.4 & 59.44 & -2.03 \\ 4 & 4 & 3.05 & 8.53 & 43.43 & 49.28 & -5.84 \\ 16 & 4 & 3.05 & 8.53 & 50.04 & 53.34 & -3.3 \\ 5 & 4 & 3.05 & 8.53 & 52.07 & 57.66 & -5.59 \\ 14 & 4 & 3.05 & 8.53 & 57.15 & 58.42 & -1.27 \\ 4 & 4 & 3.05 & 9.75 & 41.66 & 45.97 & -4.32 \\ 16 & 4 & 3.05 & 9.75 & 48.77 & 46.23 & 2.54 \\ 5 & 4 & 3.05 & 9.75 & 56.64 & 57.4 & -0.76 \\ 14 & 4 & 3.05 & 9.75 & 56.9 & 59.18 & -2.29 \\ 16 & 4 & 3.05 & 10.97 & 45.47 & 44.7 & 0.76 \\ 14 & 4 & 3.05 & 10.97 & 53.59 & 59.69 & -6.1 \\ 16 & 4 & 3.05 & 12.19 & 52.58 & 43.18 & 9.4 \\ 14 & 4 & 3.05 & 12.19 & 50.8 & 55.12 & -4.32 \\ 14 & 4 & 3.05 & 13.41 & 51.56 & 51.05 & 0.51 \\ 14 & 4 & 3.05 & 14.63 & 48.26 & 50.8 & -2.54 \\ 14 & 4 & 3.05 & 15.85 & 46.74 & 47.75 & -1.02 \\ 14 & 4 & 3.05 & 17.07 & 46.48 & 45.72 & 0.76 \\ 4 & 4 & 6.1 & 1.37 & 56.9 & 55.37 & 1.52 \\ 16 & 4 & 6.1 & 1.37 & 61.21 & 60.45 & 0.76 \\ 5 & 4 & 6.1 & 1.37 & 61.72 & 61.21 & 0.51 \\ 14 & 4 & 6.1 & 1.37 & 69.6 & 66.29 & 3.3 \\ 4 & 4 & 6.1 & 2.44 & 53.85 & 52.83 & 1.02 \\ 5 & 4 & 6.1 & 2.44 & 59.69 & 58.67 & 1.02 \\ 14 & 4 & 6.1 & 2.44 & 65.28 & 63.75 & 1.52 \\ 4 & 4 & 6.1 & 3.66 & 52.58 & 51.82 & 0.76 \\ 16 & 4 & 6.1 & 3.66 & 55.88 & 54.86 & 1.02 \\ 5 & 4 & 6.1 & 3.66 & 57.91 & 57.91 & 0 \\ 14 & 4 & 6.1 & 3.66 & 61.72 & 62.48 & -0.76 \\ 4 & 4 & 6.1 & 4.88 & 50.8 & 51.05 & -0.25 \\ 16 & 4 & 6.1 & 4.88 & 54.86 & 53.34 & 1.52 \\ 5 & 4 & 6.1 & 4.88 & 55.12 & 56.13 & -1.02 \\ 14 & 4 & 6.1 & 4.88 & 59.44 & 60.96 & -1.52 \\ 16 & 4 & 6.1 & 6.1 & 54.1 & 52.58 & 1.52 \\ 4 & 4 & 6.1 & 6.1 & 49.28 & 52.58 & -3.3 \\ 5 & 4 & 6.1 & 6.1 & 54.86 & 54.61 & 0.25 \\ 14 & 4 & 6.1 & 6.1 & 60.2 & 60.96 & -0.76 \\ 4 & 4 & 6.1 & 7.32 & 48.51 & 49.53 & -1.02 \\ 16 & 4 & 6.1 & 7.32 & 52.83 & 52.07 & 0.76 \\ 5 & 4 & 6.1 & 7.32 & 54.36 & 56.64 & -2.29 \\ 14 & 4 & 6.1 & 7.32 & 57.66 & 59.44 & -1.78 \\ 4 & 4 & 6.1 & 8.53 & 46.99 & 49.28 & -2.29 \\ 16 & 4 & 6.1 & 8.53 & 50.55 & 53.34 & -2.79 \\ 5 & 4 & 6.1 & 8.53 & 55.37 & 57.66 & -2.29 \\ 14 & 4 & 6.1 & 8.53 & 56.13 & 58.42 & -2.29 \\ 11 & 4 & 6.1 & 9.75 & 43.18 & 44.45 & -1.27 \\ 4 & 4 & 6.1 & 9.75 & 41.4 & 45.97 & -4.57 \\ 16 & 4 & 6.1 & 9.75 & 49.28 & 46.23 & 3.05 \\ 5 & 4 & 6.1 & 9.75 & 53.09 & 57.4 & -4.32 \\ 14 & 4 & 6.1 & 9.75 & 55.37 & 59.18 & -3.81 \\ 16 & 4 & 6.1 & 10.97 & 50.8 & 44.7 & 6.1\end{array}$




$\begin{array}{lllllll}11 & 4 & 6.1 & 10.97 & 41.66 & 44.96 & -3.3 \\ 5 & 4 & 6.1 & 10.97 & 49.28 & 54.86 & -5.59 \\ 14 & 4 & 6.1 & 10.97 & 59.18 & 59.69 & -0.51 \\ 11 & 4 & 6.1 & 12.19 & 38.61 & 36.83 & 1.78 \\ 16 & 4 & 6.1 & 12.19 & 44.96 & 43.18 & 1.78 \\ 5 & 4 & 6.1 & 12.19 & 48.26 & 47.75 & 0.51 \\ 14 & 4 & 6.1 & 12.19 & 57.15 & 55.12 & 2.03 \\ 11 & 4 & 6.1 & 13.41 & 36.07 & 34.8 & 1.27 \\ 16 & 4 & 6.1 & 13.41 & 43.69 & 42.42 & 1.27 \\ 5 & 4 & 6.1 & 13.41 & 44.96 & 46.48 & -1.52 \\ 14 & 4 & 6.1 & 13.41 & 50.8 & 51.05 & -0.25 \\ 11 & 4 & 6.1 & 14.63 & 36.32 & 34.04 & 2.29 \\ 16 & 4 & 6.1 & 14.63 & 40.89 & 37.85 & 3.05 \\ 5 & 4 & 6.1 & 14.63 & 40.64 & 46.74 & -6.1 \\ 14 & 4 & 6.1 & 14.63 & 47.75 & 50.8 & -3.05 \\ 11 & 4 & 6.1 & 15.85 & 35.81 & 30.73 & 5.08 \\ 5 & 4 & 6.1 & 15.85 & 36.32 & 39.12 & -2.79 \\ 14 & 4 & 6.1 & 15.85 & 47.5 & 47.75 & -0.25 \\ 11 & 4 & 6.1 & 17.07 & 35.31 & 32.51 & 2.79 \\ 14 & 4 & 6.1 & 17.07 & 42.67 & 45.72 & -3.05 \\ 14 & 4 & 6.1 & 18.29 & 44.7 & 34.29 & 10.41 \\ 4 & 4 & 9.14 & 1.37 & 56.64 & 55.37 & 1.27 \\ 16 & 4 & 9.14 & 1.37 & 59.44 & 60.45 & -1.02 \\ 5 & 4 & 9.14 & 1.37 & 60.96 & 61.21 & -0.25 \\ 14 & 4 & 9.14 & 1.37 & 69.6 & 66.29 & 3.3 \\ 4 & 4 & 9.14 & 2.44 & 54.86 & 52.83 & 2.03 \\ 5 & 4 & 9.14 & 2.44 & 59.44 & 58.67 & 0.76 \\ 14 & 4 & 9.14 & 2.44 & 65.79 & 63.75 & 2.03 \\ 4 & 4 & 9.14 & 3.66 & 55.37 & 51.82 & 3.56 \\ 16 & 4 & 9.14 & 3.66 & 57.4 & 54.86 & 2.54 \\ 5 & 4 & 9.14 & 3.66 & 57.91 & 57.91 & 0 \\ 14 & 4 & 9.14 & 3.66 & 61.72 & 62.48 & -0.76 \\ 4 & 4 & 9.14 & 4.88 & 54.36 & 51.05 & 3.3 \\ 16 & 4 & 9.14 & 4.88 & 54.1 & 53.34 & 0.76 \\ 5 & 4 & 9.14 & 4.88 & 55.63 & 56.13 & -0.51 \\ 14 & 4 & 9.14 & 4.88 & 60.45 & 60.96 & -0.51 \\ 16 & 4 & 9.14 & 6.1 & 53.59 & 52.58 & 1.02 \\ 4 & 4 & 9.14 & 6.1 & 49.53 & 52.58 & -3.05 \\ 5 & 4 & 9.14 & 6.1 & 54.36 & 54.61 & -0.25 \\ 14 & 4 & 9.14 & 6.1 & 59.18 & 60.96 & -1.78 \\ 4 & 4 & 9.14 & 7.32 & 49.78 & 49.53 & 0.25 \\ 16 & 4 & 9.14 & 7.32 & 51.82 & 52.07 & -0.25 \\ 5 & 4 & 9.14 & 7.32 & 54.1 & 56.64 & -2.54 \\ 14 & 4 & 9.14 & 7.32 & 57.4 & 59.44 & -2.03 \\ 4 & 4 & 9.14 & 8.53 & 48.77 & 49.28 & -0.51 \\ 16 & 4 & 9.14 & 8.53 & 52.83 & 53.34 & -0.51 \\ 5 & 4 & 9.14 & 8.53 & 54.86 & 57.66 & -2.79 \\ 14 & 4 & 9.14 & 8.53 & 56.39 & 58.42 & -2.03 \\ 11 & 4 & 9.14 & 9.75 & 48.77 & 44.45 & 4.32 \\ 4 & 4 & 9.14 & 9.75 & 47.24 & 45.97 & 1.27 \\ 16 & 4 & 9.14 & 9.75 & 53.59 & 46.23 & 7.37 \\ 5 & 4 & 9.14 & 9.75 & 54.1 & 57.4 & -3.3 \\ 14 & 4 & 9.14 & 9.75 & 56.39 & 59.18 & -2.79 \\ 4 & 4 & 9.14 & 10.97 & 43.69 & 43.18 & 0.51\end{array}$




\begin{tabular}{|c|c|c|c|c|c|c|}
\hline 16 & 4 & 9.14 & 10.97 & 44.2 & 44.7 & -0.51 \\
\hline 11 & 4 & 9.14 & 10.97 & 42.93 & 44.96 & -2.03 \\
\hline 5 & 4 & 9.14 & 10.97 & 50.8 & 54.86 & -4.06 \\
\hline 14 & 4 & 9.14 & 10.97 & 55.88 & 59.69 & -3.81 \\
\hline 11 & 4 & 9.14 & 12.19 & 40.39 & 36.83 & 3.56 \\
\hline 16 & 4 & 9.14 & 12.19 & 44.7 & 43.18 & 1.52 \\
\hline 5 & 4 & 9.14 & 12.19 & 52.58 & 47.75 & 4.83 \\
\hline 14 & 4 & 9.14 & 12.19 & 50.55 & 55.12 & -4.57 \\
\hline 11 & 4 & 9.14 & 13.41 & 36.07 & 34.8 & 1.27 \\
\hline 16 & 4 & 9.14 & 13.41 & 43.94 & 42.42 & 1.52 \\
\hline 5 & 4 & 9.14 & 13.41 & 46.99 & 46.48 & 0.51 \\
\hline 14 & 4 & 9.14 & 13.41 & 49.02 & 51.05 & -2.03 \\
\hline 11 & 4 & 9.14 & 14.63 & 35.31 & 34.04 & 1.27 \\
\hline 16 & 4 & 9.14 & 14.63 & 39.88 & 37.85 & 2.03 \\
\hline 5 & 4 & 9.14 & 14.63 & 41.15 & 46.74 & -5.59 \\
\hline 14 & 4 & 9.14 & 14.63 & 46.99 & 50.8 & -3.81 \\
\hline 11 & 4 & 9.14 & 15.85 & 34.29 & 30.73 & 3.56 \\
\hline 16 & 4 & 9.14 & 15.85 & 43.94 & 33.78 & 10.16 \\
\hline 5 & 4 & 9.14 & 15.85 & 36.07 & 39.12 & -3.05 \\
\hline 14 & 4 & 9.14 & 15.85 & 42.16 & 47.75 & -5.59 \\
\hline 11 & 4 & 9.14 & 17.07 & 30.99 & 32.51 & -1.52 \\
\hline 14 & 4 & 9.14 & 17.07 & 40.13 & 45.72 & -5.59 \\
\hline 14 & 4 & 9.14 & 18.29 & 33.02 & 34.29 & -1.27 \\
\hline 14 & 4 & 9.14 & 19.51 & 30.23 & 32.77 & -2.54 \\
\hline 4 & 4 & 12.19 & 1.37 & 57.66 & 55.37 & 2.29 \\
\hline 16 & 4 & 12.19 & 1.37 & 59.44 & 60.45 & -1.02 \\
\hline 5 & 4 & 12.19 & 1.37 & 60.71 & 61.21 & -0.51 \\
\hline 14 & 4 & 12.19 & 1.37 & 67.56 & 66.29 & 1.27 \\
\hline 4 & 4 & 12.19 & 2.44 & 55.63 & 52.83 & 2.79 \\
\hline 5 & 4 & 12.19 & 2.44 & 59.69 & 58.67 & 1.02 \\
\hline 14 & 4 & 12.19 & 2.44 & 64.77 & 63.75 & 1.02 \\
\hline 4 & 4 & 12.19 & 3.66 & 53.85 & 51.82 & 2.03 \\
\hline 16 & 4 & 12.19 & 3.66 & 57.66 & 54.86 & 2.79 \\
\hline 5 & 4 & 12.19 & 3.66 & 57.4 & 57.91 & -0.51 \\
\hline 14 & 4 & 12.19 & 3.66 & 61.72 & 62.48 & -0.76 \\
\hline 4 & 4 & 12.19 & 4.88 & 52.32 & 51.05 & 1.27 \\
\hline 16 & 4 & 12.19 & 4.88 & 51.31 & 53.34 & -2.03 \\
\hline 5 & 4 & 12.19 & 4.88 & 55.12 & 56.13 & -1.02 \\
\hline 14 & 4 & 12.19 & 4.88 & 59.44 & 60.96 & -1.52 \\
\hline 16 & 4 & 12.19 & 6.1 & 52.58 & 52.58 & 0 \\
\hline 4 & 4 & 12.19 & 6.1 & 52.58 & 52.58 & 0 \\
\hline 5 & 4 & 12.19 & 6.1 & 54.61 & 54.61 & 0 \\
\hline 14 & 4 & 12.19 & 6.1 & 59.18 & 60.96 & -1.78 \\
\hline 4 & 4 & 12.19 & 7.32 & 49.78 & 49.53 & 0.25 \\
\hline 16 & 4 & 12.19 & 7.32 & 51.56 & 52.07 & -0.51 \\
\hline 5 & 4 & 12.19 & 7.32 & 54.86 & 56.64 & -1.78 \\
\hline 14 & 4 & 12.19 & 7.32 & 57.15 & 59.44 & -2.29 \\
\hline 4 & 4 & 12.19 & 8.53 & 49.53 & 49.28 & 0.25 \\
\hline 16 & 4 & 12.19 & 8.53 & 55.12 & 53.34 & 1.78 \\
\hline 5 & 4 & 12.19 & 8.53 & 56.39 & 57.66 & -1.27 \\
\hline 14 & 4 & 12.19 & 8.53 & 55.63 & 58.42 & -2.79 \\
\hline 11 & 4 & 12.19 & 9.75 & 44.45 & 44.45 & 0 \\
\hline 4 & 4 & 12.19 & 9.75 & 46.48 & 45.97 & 0.51 \\
\hline 16 & 4 & 12.19 & 9.75 & 56.9 & 46.23 & 10.67 \\
\hline
\end{tabular}




\begin{tabular}{|c|c|c|c|c|c|c|}
\hline 5 & 4 & 12.19 & 9.75 & 56.13 & 57.4 & -1.27 \\
\hline 14 & 4 & 12.19 & 9.75 & 55.63 & 59.18 & -3.56 \\
\hline 4 & 4 & 12.19 & 10.97 & 43.69 & 43.18 & 0.51 \\
\hline 16 & 4 & 12.19 & 10.97 & 47.24 & 44.7 & 2.54 \\
\hline 11 & 4 & 12.19 & 10.97 & 44.45 & 44.96 & -0.51 \\
\hline 5 & 4 & 12.19 & 10.97 & 51.82 & 54.86 & -3.05 \\
\hline 14 & 4 & 12.19 & 10.97 & 56.39 & 59.69 & -3.3 \\
\hline 11 & 4 & 12.19 & 12.19 & 39.62 & 36.83 & 2.79 \\
\hline 16 & 4 & 12.19 & 12.19 & 45.47 & 43.18 & 2.29 \\
\hline 4 & 4 & 12.19 & 12.19 & 40.39 & 43.94 & -3.56 \\
\hline 5 & 4 & 12.19 & 12.19 & 51.31 & 47.75 & 3.56 \\
\hline 14 & 4 & 12.19 & 12.19 & 53.09 & 55.12 & -2.03 \\
\hline 11 & 4 & 12.19 & 13.41 & 36.83 & 34.8 & 2.03 \\
\hline 16 & 4 & 12.19 & 13.41 & 44.7 & 42.42 & 2.29 \\
\hline 5 & 4 & 12.19 & 13.41 & 46.48 & 46.48 & 0 \\
\hline 14 & 4 & 12.19 & 13.41 & 49.53 & 51.05 & -1.52 \\
\hline 11 & 4 & 12.19 & 14.63 & 37.85 & 34.04 & 3.81 \\
\hline 16 & 4 & 12.19 & 14.63 & 41.91 & 37.85 & 4.06 \\
\hline 5 & 4 & 12.19 & 14.63 & 41.91 & 46.74 & -4.83 \\
\hline 14 & 4 & 12.19 & 14.63 & 45.72 & 50.8 & -5.08 \\
\hline 11 & 4 & 12.19 & 15.85 & 32.77 & 30.73 & 2.03 \\
\hline 16 & 4 & 12.19 & 15.85 & 41.91 & 33.78 & 8.13 \\
\hline 5 & 4 & 12.19 & 15.85 & 35.56 & 39.12 & -3.56 \\
\hline 14 & 4 & 12.19 & 15.85 & 41.66 & 47.75 & -6.1 \\
\hline 11 & 4 & 12.19 & 17.07 & 30.23 & 32.51 & -2.29 \\
\hline 16 & 4 & 12.19 & 17.07 & 34.04 & 33.53 & 0.51 \\
\hline 14 & 4 & 12.19 & 17.07 & 34.8 & 45.72 & -10.92 \\
\hline 4 & 4 & 15.24 & 1.37 & 57.91 & 55.37 & 2.54 \\
\hline 16 & 4 & 15.24 & 1.37 & 57.4 & 60.45 & -3.05 \\
\hline 5 & 4 & 15.24 & 1.37 & 60.96 & 61.21 & -0.25 \\
\hline 14 & 4 & 15.24 & 1.37 & 68.07 & 66.29 & 1.78 \\
\hline 4 & 4 & 15.24 & 2.44 & 55.37 & 52.83 & 2.54 \\
\hline 5 & 4 & 15.24 & 2.44 & 57.91 & 58.67 & -0.76 \\
\hline 14 & 4 & 15.24 & 2.44 & 62.99 & 63.75 & -0.76 \\
\hline 4 & 4 & 15.24 & 3.66 & 54.86 & 51.82 & 3.05 \\
\hline 16 & 4 & 15.24 & 3.66 & 57.15 & 54.86 & 2.29 \\
\hline 5 & 4 & 15.24 & 3.66 & 57.66 & 57.91 & -0.25 \\
\hline 14 & 4 & 15.24 & 3.66 & 61.72 & 62.48 & -0.76 \\
\hline 4 & 4 & 15.24 & 4.88 & 51.82 & 51.05 & 0.76 \\
\hline 16 & 4 & 15.24 & 4.88 & 53.09 & 53.34 & -0.25 \\
\hline 5 & 4 & 15.24 & 4.88 & 55.12 & 56.13 & -1.02 \\
\hline 14 & 4 & 15.24 & 4.88 & 59.94 & 60.96 & -1.02 \\
\hline 16 & 4 & 15.24 & 6.1 & 52.58 & 52.58 & 0 \\
\hline 4 & 4 & 15.24 & 6.1 & 51.82 & 52.58 & -0.76 \\
\hline 5 & 4 & 15.24 & 6.1 & 54.36 & 54.61 & -0.25 \\
\hline 14 & 4 & 15.24 & 6.1 & 58.67 & 60.96 & -2.29 \\
\hline 4 & 4 & 15.24 & 7.32 & 50.04 & 49.53 & 0.51 \\
\hline 16 & 4 & 15.24 & 7.32 & 50.8 & 52.07 & -1.27 \\
\hline 5 & 4 & 15.24 & 7.32 & 54.86 & 56.64 & -1.78 \\
\hline 14 & 4 & 15.24 & 7.32 & 56.39 & 59.44 & -3.05 \\
\hline 4 & 4 & 15.24 & 8.53 & 47.75 & 49.28 & -1.52 \\
\hline 16 & 4 & 15.24 & 8.53 & 54.86 & 53.34 & 1.52 \\
\hline 5 & 4 & 15.24 & 8.53 & 55.63 & 57.66 & -2.03 \\
\hline 14 & 4 & 15.24 & 8.53 & 55.63 & 58.42 & -2.79 \\
\hline
\end{tabular}




$\begin{array}{lllllll}11 & 4 & 15.24 & 9.75 & 43.94 & 44.45 & -0.51 \\ 4 & 4 & 15.24 & 9.75 & 44.2 & 45.97 & -1.78 \\ 16 & 4 & 15.24 & 9.75 & 54.86 & 46.23 & 8.64 \\ 5 & 4 & 15.24 & 9.75 & 54.1 & 57.4 & -3.3 \\ 14 & 4 & 15.24 & 9.75 & 55.63 & 59.18 & -3.56 \\ 4 & 4 & 15.24 & 10.97 & 42.16 & 43.18 & -1.02 \\ 16 & 4 & 15.24 & 10.97 & 44.2 & 44.7 & -0.51 \\ 11 & 4 & 15.24 & 10.97 & 44.7 & 44.96 & -0.25 \\ 5 & 4 & 15.24 & 10.97 & 51.56 & 54.86 & -3.3 \\ 14 & 4 & 15.24 & 10.97 & 53.09 & 59.69 & -6.6 \\ 11 & 4 & 15.24 & 12.19 & 40.89 & 36.83 & 4.06 \\ 16 & 4 & 15.24 & 12.19 & 45.72 & 43.18 & 2.54 \\ 5 & 4 & 15.24 & 12.19 & 51.31 & 47.75 & 3.56 \\ 14 & 4 & 15.24 & 12.19 & 52.32 & 55.12 & -2.79 \\ 11 & 4 & 15.24 & 13.41 & 37.85 & 34.8 & 3.05 \\ 16 & 4 & 15.24 & 13.41 & 41.15 & 42.42 & -1.27 \\ 5 & 4 & 15.24 & 13.41 & 47.24 & 46.48 & 0.76 \\ 14 & 4 & 15.24 & 13.41 & 50.29 & 51.05 & -0.76 \\ 11 & 4 & 15.24 & 14.63 & 36.83 & 34.04 & 2.79 \\ 16 & 4 & 15.24 & 14.63 & 39.62 & 37.85 & 1.78 \\ 5 & 4 & 15.24 & 14.63 & 43.43 & 46.74 & -3.3 \\ 14 & 4 & 15.24 & 14.63 & 47.75 & 50.8 & -3.05 \\ 11 & 4 & 15.24 & 15.85 & 34.54 & 30.73 & 3.81 \\ 16 & 4 & 15.24 & 15.85 & 35.31 & 33.78 & 1.52 \\ 5 & 4 & 15.24 & 15.85 & 38.1 & 39.12 & -1.02 \\ 14 & 4 & 15.24 & 15.85 & 44.2 & 47.75 & -3.56 \\ 11 & 4 & 15.24 & 17.07 & 31.75 & 32.51 & -0.76 \\ 16 & 4 & 15.24 & 17.07 & 30.99 & 33.53 & -2.54 \\ 14 & 4 & 15.24 & 17.07 & 38.35 & 45.72 & -7.37 \\ 14 & 4 & 15.24 & 18.29 & 35.05 & 34.29 & 0.76 \\ 14 & 4 & 15.24 & 19.51 & 30.48 & 32.77 & -2.29 \\ ; & & & & & & \\ \text { run; } & & & & & & \end{array}$

ALL INCLUSIVE MODEL

proc mixed data $=\mathrm{tm}$;

class nomdist tree nomdiam height;

model diff=nomdist nomdiam height height*nomdist/ddfm=satterth;

repeated/type $=\mathrm{cs}$ sub=tree(nomdist) $\mathrm{rcorr}$;

lsmeans nomdist nomdiam height;

contrast ' $10 \mathrm{v}$. all' nomdist $4-1-1-1-1$;

contrast ' $20 \mathrm{v}$. all' nomdist $-14-1-1-1$;

contrast ' $30 \mathrm{v}$. all' nomdist $-1-14-1-1$;

contrast '40 v. all' nomdist $-1-1-14-1$;

contrast ' $50 \mathrm{v}$. all' nomdist $-1-1-1-14$;

contrast '6-12 v. all' nomdiam $3-1-1-1$;

contrast '12-18 v. all' nomdiam -1 $3-1-1$;

contrast ' $18-24 \mathrm{v}$. all' nomdiam $-1-13-1$;

contrast '>24 v. all' nomdiam -1 -1 -13;

title1 'repeated measures mixed model(cs)';

title2 'nomdist nomdiam height $\mathrm{h}$ *ndist';

run; 


\section{OUTPUT}

repeated measures mixed model

The MIXED Procedure

Class Level Information

Class Levels Values

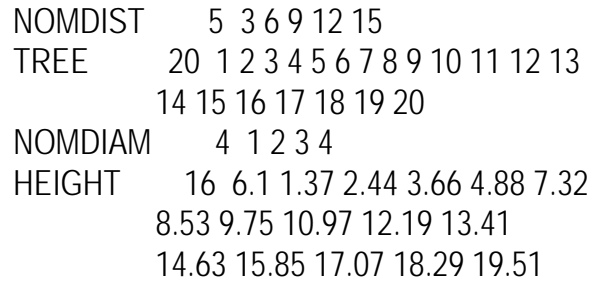

REML Estimation Iteration History

Iteration Evaluations Objective Criterion

$0 \quad 12816.1204574$

$122643.6861542 \quad 0.00000802$

$\begin{array}{llll}2 & 1 & 2643.6753491 & 0.00000001\end{array}$

Convergence criteria met.

R Correlation Matrix for TREE(NOMDIST) 13

$\begin{array}{lrrrrrrl}\text { Row } & \text { COL1 } & \text { COL2 } & \text { COL3 } & \text { COL4 } & \text { COL5 } & \text { COL6 } \\ & & & & & & \\ 1 & 1.00000000 & 0.27836487 & 0.27836487 & 0.27836487 & 0.27836487 & 0.27836487 \\ 2 & 0.27836487 & 1.00000000 & 0.27836487 & 0.27836487 & 0.27836487 & 0.27836487 \\ 3 & 0.27836487 & 0.27836487 & 1.00000000 & 0.27836487 & 0.27836487 & 0.27836487 \\ 4 & 0.27836487 & 0.27836487 & 0.27836487 & 1.00000000 & 0.27836487 & 0.27836487 \\ 5 & 0.27836487 & 0.27836487 & 0.27836487 & 0.27836487 & 1.00000000 & 0.27836487 \\ 6 & 0.27836487 & 0.27836487 & 0.27836487 & 0.27836487 & 0.27836487 & 1.00000000 \\ 7 & 0.27836487 & 0.27836487 & 0.27836487 & 0.27836487 & 0.27836487 & 0.27836487 \\ 8 & 0.27836487 & 0.27836487 & 0.27836487 & 0.27836487 & 0.27836487 & 0.27836487 \\ 9 & 0.27836487 & 0.27836487 & 0.27836487 & 0.27836487 & 0.27836487 & 0.27836487\end{array}$

R Correlation Matrix for TREE(NOMDIST) 13

$\begin{array}{ccl}\text { COL7 } & \text { COL8 } & \text { COL9 } \\ & & \\ 0.27836487 & 0.27836487 & 0.27836487 \\ 0.27836487 & 0.27836487 & 0.27836487 \\ 0.27836487 & 0.27836487 & 0.27836487 \\ 0.27836487 & 0.27836487 & 0.27836487 \\ 0.27836487 & 0.27836487 & 0.27836487\end{array}$


$\begin{array}{lll}0.27836487 & 0.27836487 & 0.27836487\end{array}$

repeated measures mixed model

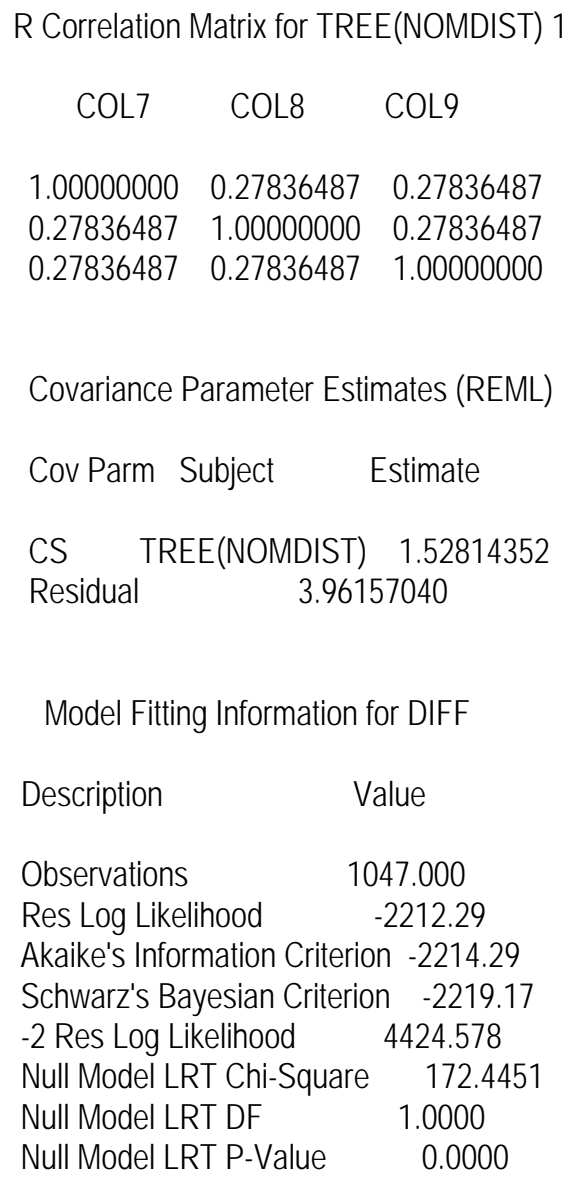

Tests of Fixed Effects

Source NDF DDF Type III F Pr > F

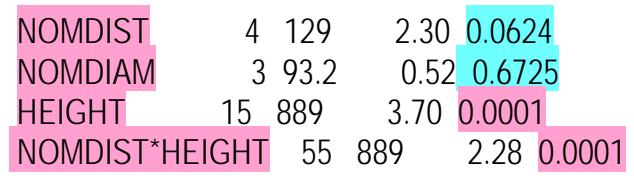

CONTRAST Statement Results

\begin{tabular}{|c|c|c|}
\hline Source & NDF DDF & $\mathrm{FPr}>\mathrm{F}$ \\
\hline $10 \mathrm{v}$. all & . & . \\
\hline 20 v. all & . & . \\
\hline $30 \mathrm{v}$. all & . & . \\
\hline $40 \mathrm{v}$. all & . & . \\
\hline $50 \mathrm{v}$. all & $. \quad . \quad$. & \\
\hline 6-12 v. all & 198.1 & $0 \quad 0.2751$ \\
\hline
\end{tabular}


CONTRAST Statement Results

$\begin{array}{llll}\text { Source } & \text { NDF DDF } & F \text { Pr }>F \\ & & & \\ \text { 12-18 v. all } & 191.5 & 0.01 & 0.9368 \\ \text { 18-24 v. all } & 191.2 & 0.91 & 0.3425 \\ \text { >24 v. all } & 193.1 & 0.03 & 0.8614\end{array}$

Least Squares Means

Effect NOMDIST NOMDIAM HEIGHT LSMEAN Std Error DF $\mathrm{t}$ Pr $>|t|$

NOMDIST 3

NOMDIST 6

NOMDIST 9

NOMDIST 12

NOMDIST 15

NOMDIAM 1

NOMDIAM 2

NOMDIAM 3

NOMDIAM 4

HEIGHT

HEIGHT

HEIGHT

HEIGHT

HEIGHT

HEIGHT

HEIGHT

HEIGHT

HEIGHT

HEIGHT

HEIGHT

HEIGHT

HEIGHT

HEIGHT

HEIGHT

HEIGHT

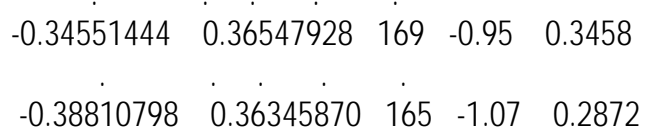

\section{HEIGHT ONLY MODEL}

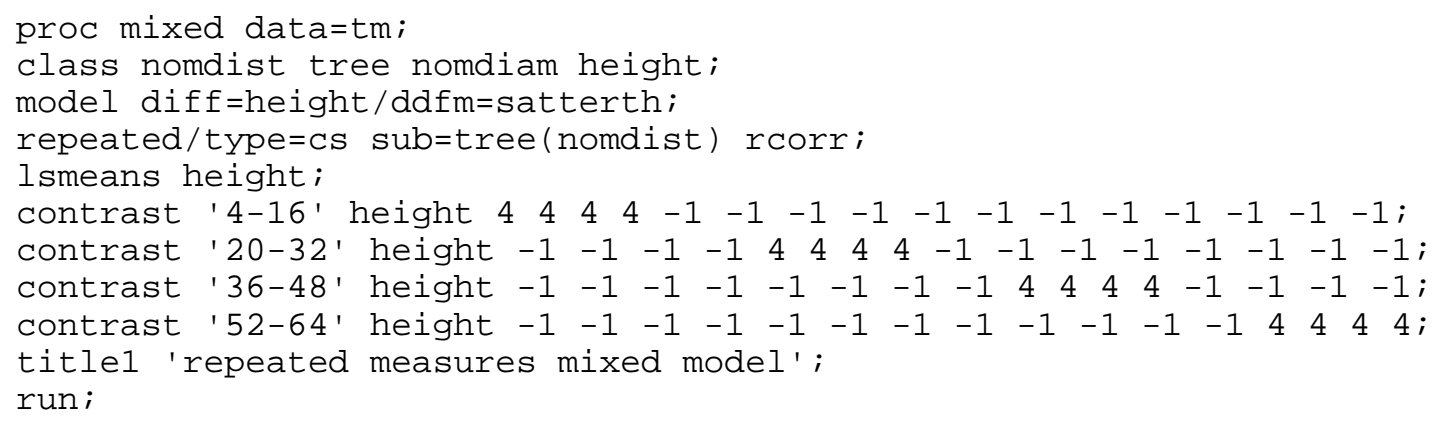




\section{OUTPUT}

repeated measures mixed model

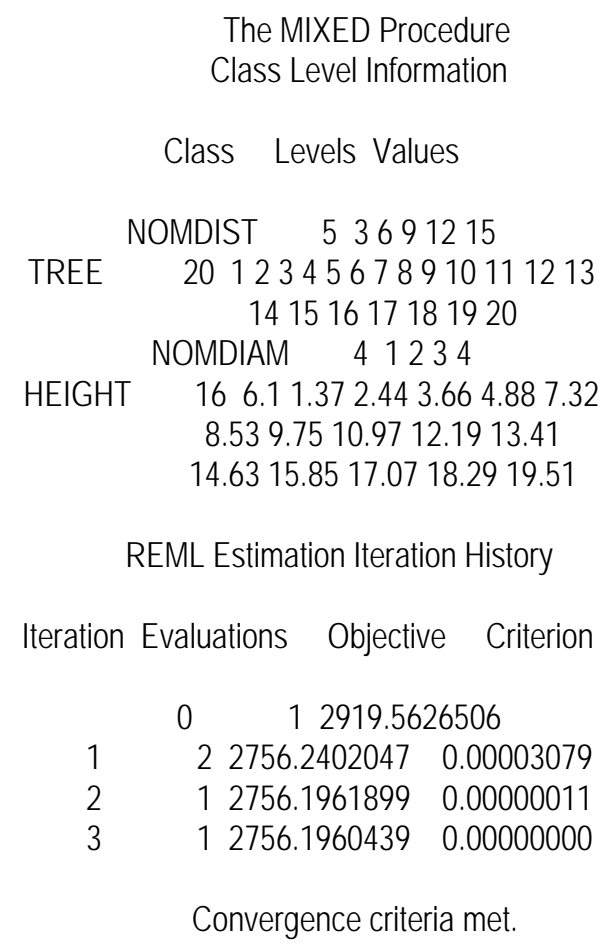

R Correlation Matrix for TREE(NOMDIST) 13

\begin{tabular}{|c|c|c|c|c|c|c|}
\hline & Row & 1 & $\operatorname{coL} 3$ & COL4 & COL5 & COL6 \\
\hline & 1.00000000 & 0.25 & 0.25 & 02 & 02 & 0.25434702 \\
\hline & 0.25434702 & 1.00000000 & 0.25434702 & 0.25434702 & 0.25434702 & 0.25434702 \\
\hline & 0.25434702 & 0.25434702 & 1.00000000 & 0.25434702 & 0.25434702 & 0.25434702 \\
\hline & 0.25434702 & 0.25434702 & 0.25434702 & 1.00000000 & 0.25434702 & 0.2543470 \\
\hline & 0.25434702 & 0.25434702 & 0.25434702 & 0.25434702 & 1.00000000 & 0.2543470 \\
\hline & 0.25434702 & 0.25434702 & 0.25434702 & 0.25434702 & 0.25434702 & 1.00000000 \\
\hline & 0.25434702 & 0.25434702 & 0.25434702 & 0.25434702 & 0.25434702 & 0.25434702 \\
\hline & 0.25434702 & 0.25434702 & 0.25434702 & 0.25434702 & 0.25434702 & 0.25434702 \\
\hline & 0.25434702 & 0.25434702 & 0.25434702 & 0.25434702 & 0.25434702 & 0.25434702 \\
\hline
\end{tabular}

R Correlation Matrix for TREE(NOMDIST) 13

$\begin{array}{rrc}\text { COL7 } & \text { COL8 } & \text { COL9 } \\ & & \\ 0.25434702 & 0.25434702 & 0.25434702 \\ 0.25434702 & 0.25434702 & 0.25434702 \\ 0.25434702 & 0.25434702 & 0.25434702 \\ 0.25434702 & 0.25434702 & 0.25434702 \\ 0.25434702 & 0.25434702 & 0.25434702 \\ \text { repeated measures mixed model } & \end{array}$


R Correlation Matrix for TREE(NOMDIST) 13

$\begin{array}{rrc}\text { COL7 } & \text { COL8 } & \text { COL9 } \\ & & \\ 0.25434702 & 0.25434702 & 0.25434702 \\ 1.00000000 & 0.25434702 & 0.25434702 \\ 0.25434702 & 1.00000000 & 0.25434702 \\ 0.25434702 & 0.25434702 & 1.00000000\end{array}$

Covariance Parameter Estimates (REML)

Cov Parm Subject Estimate

CS TREE(NOMDIST) 1.50360473

Residual $\quad 4.40802235$

Model Fitting Information for DIFF

Description Value

Observations $\quad 1047.000$

Res Log Likelihood $\quad-2335.63$

Akaike's Information Criterion -2337.63

Schwarz's Bayesian Criterion $\quad-2342.58$

-2 Res Log Likelihood $\quad 4671.264$

Null Model LRT Chi-Square 163.3666

Null Model LRT DF $\quad 1.0000$

Null Model LRT P-Value $\quad 0.0000$

Tests of Fixed Effects

Source NDF DDF Type III F Pr > F

NOMDIST $496.5 \quad 0.260 .9057$

CONTRAST Statement Results

Source $\quad$ NDF DDF $\quad F$ Pr $>F$

$10 \mathrm{v}$. all $\quad 11020.730 .3936$

$20 \mathrm{v}$. all $\quad 196.10 .190 .6655$

$30 \mathrm{v}$. all $\quad 195.10 .110 .7377$

$40 \mathrm{v}$. all $\quad 194.4 \quad 0.170 .6815$

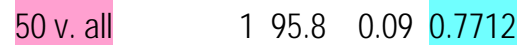

repeated measures mixed model

3

Least Squares Means

Effect NOMDIST LSMEAN Std Error DF $\mathrm{t} P r>|t|$ 


\begin{tabular}{|c|c|c|c|c|c|}
\hline NOMDIST 3 & -0.49381713 & 0.32553251 & 103 & -1.52 & 0.1323 \\
\hline NOMDIST 6 & -0.12574658 & 0.31119261 & 96 & -0.40 & 0.6871 \\
\hline NOMDIST 9 & -0.15328922 & 0.30983769 & 94.6 & -0.49 & 0.6219 \\
\hline NOMDIST 12 & -0.13245874 & 0.30895720 & 93.6 & -0.43 & 0.6691 \\
\hline NOMDIST 15 & -0.32795224 & 0.31105666 & 95.5 & -1.05 & 0.2944 \\
\hline
\end{tabular}

The MIXED Procedure
Class Level Information
Class Levels Values
NOMDIST 53691215
TREE $\quad 2012345678910111213$
14151617181920
NOMDIAM 41234
HEIGHT 6.11 .372 .443 .664 .887 .32
8.539 .7510 .9712 .1913 .41
14.6315 .8517 .0718 .2919 .51

REML Estimation Iteration History

Iteration Evaluations Objective Criterion

\begin{tabular}{|c|c|c|}
\hline & 12813.29 & 10667 \\
\hline 1 & 22639.0225115 & 0.00001409 \\
\hline 2 & 12639.0034465 & 0.00000002 \\
\hline 3 & 12639.0034179 & 0.00000000 \\
\hline
\end{tabular}

R Correlation Matrix for TREE(NOMDIST) 13

$\begin{array}{llllcccl} & \text { RoW } & \text { COL1 } & \text { COL2 } & \text { COL3 } & \text { COL4 } & \text { COL5 } & \text { COL6 } \\ & & & & & & \\ 1 & 1.00000000 & 0.27483805 & 0.27483805 & 0.27483805 & 0.27483805 & 0.27483805 \\ 2 & 0.27483805 & 1.00000000 & 0.27483805 & 0.27483805 & 0.27483805 & 0.27483805 \\ 3 & 0.27483805 & 0.27483805 & 1.00000000 & 0.27483805 & 0.27483805 & 0.27483805 \\ 4 & 0.27483805 & 0.27483805 & 0.27483805 & 1.00000000 & 0.27483805 & 0.27483805 \\ 5 & 0.27483805 & 0.27483805 & 0.27483805 & 0.27483805 & 1.00000000 & 0.27483805 \\ 6 & 0.27483805 & 0.27483805 & 0.27483805 & 0.27483805 & 0.27483805 & 1.00000000 \\ 7 & 0.27483805 & 0.27483805 & 0.27483805 & 0.27483805 & 0.27483805 & 0.27483805 \\ 8 & 0.27483805 & 0.27483805 & 0.27483805 & 0.27483805 & 0.27483805 & 0.27483805 \\ 9 & 0.27483805 & 0.27483805 & 0.27483805 & 0.27483805 & 0.27483805 & 0.27483805\end{array}$

R Correlation Matrix for TREE(NOMDIST) 13

$$
\begin{array}{rrc}
\text { COL7 } & \text { COL8 } & \text { COL9 } \\
& & \\
0.27483805 & 0.27483805 & 0.27483805
\end{array}
$$




$\begin{array}{rrr}0.27483805 & 0.27483805 & 0.27483805 \\ 0.27483805 & 0.27483805 & 0.27483805 \\ 0.27483805 & 0.27483805 & 0.27483805 \\ 0.27483805 & 0.27483805 & 0.27483805 \\ \text { repeated measures mixed model } & \end{array}$

R Correlation Matrix for TREE(NOMDIST) 13

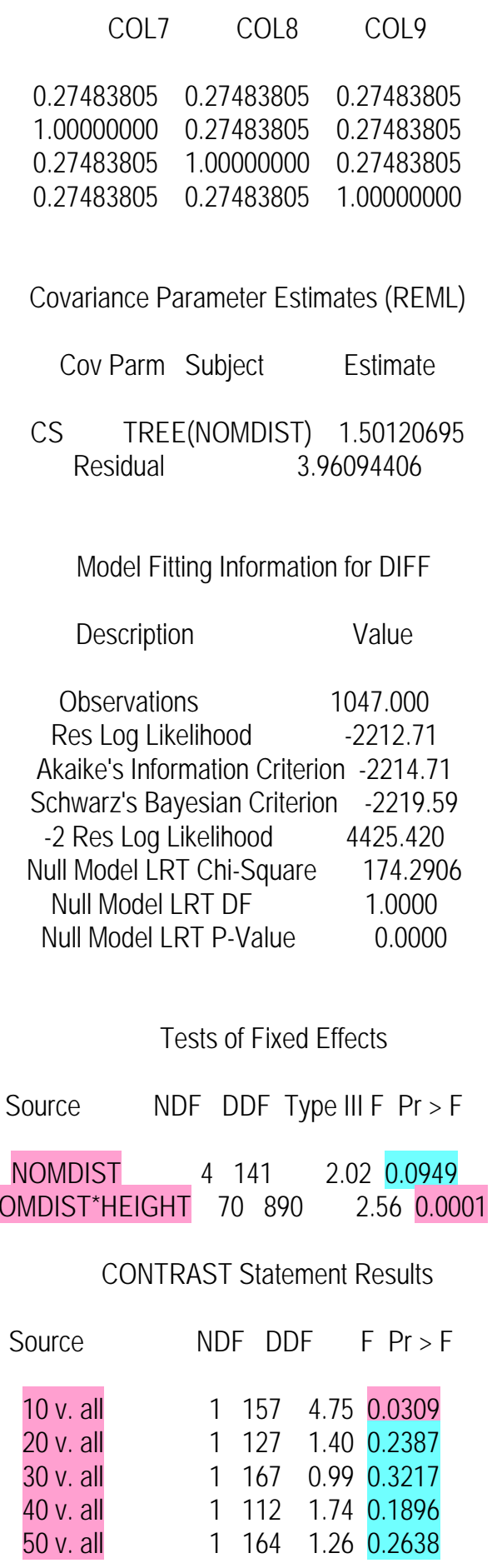


The MIXED Procedure

Class Level Information

Class Levels Values

\begin{tabular}{|c|c|}
\hline NOMDIST & 53691215 \\
\hline TREE & $\begin{array}{l}2012345678910111213 \\
14151617181920\end{array}$ \\
\hline NOMDIAM & 41234 \\
\hline HEIGHT & $\begin{array}{l}16 \quad 6.11 .372 .443 .664 .887 .32 \\
8.539 .7510 .9712 .1913 .41 \\
14.6315 .8517 .0718 .2919 .51\end{array}$ \\
\hline
\end{tabular}

REML Estimation Iteration History

Iteration Evaluations Objective Criterion

$0 \quad 12907.4975224$

$\begin{array}{llll}1 & 2 & 2732.5211865 & 0.00000654\end{array}$

$2 \quad 12732.5120886 \quad 0.00000000$

Convergence criteria met.

R Correlation Matrix for TREE(NOMDIST) 13

$\begin{array}{lccccccc}\text { Row } & \text { COL1 } & \text { COL2 } & \text { COL3 } & \text { COL4 } & \text { COL5 } & \text { COL6 } \\ & & & & & & \\ 1 & 1.00000000 & 0.26600841 & 0.26600841 & 0.26600841 & 0.26600841 & 0.26600841 \\ 2 & 0.26600841 & 1.00000000 & 0.26600841 & 0.26600841 & 0.26600841 & 0.26600841 \\ 3 & 0.26600841 & 0.26600841 & 1.00000000 & 0.26600841 & 0.26600841 & 0.26600841 \\ 4 & 0.26600841 & 0.26600841 & 0.26600841 & 1.00000000 & 0.26600841 & 0.26600841 \\ 5 & 0.26600841 & 0.26600841 & 0.26600841 & 0.26600841 & 1.00000000 & 0.26600841 \\ 6 & 0.26600841 & 0.26600841 & 0.26600841 & 0.26600841 & 0.26600841 & 1.00000000 \\ 7 & 0.26600841 & 0.26600841 & 0.26600841 & 0.26600841 & 0.26600841 & 0.26600841 \\ 8 & 0.26600841 & 0.26600841 & 0.26600841 & 0.26600841 & 0.26600841 & 0.26600841 \\ 9 & 0.26600841 & 0.26600841 & 0.26600841 & 0.26600841 & 0.26600841 & 0.26600841\end{array}$

R Correlation Matrix for TREE(NOMDIST) 13

COL7 COL8 COL9

$\begin{array}{lll}0.26600841 & 0.26600841 & 0.26600841\end{array}$

$\begin{array}{lll}0.26600841 & 0.26600841 & 0.26600841\end{array}$

$\begin{array}{lll}0.26600841 & 0.26600841 & 0.26600841\end{array}$

$\begin{array}{lll}0.26600841 & 0.26600841 & 0.26600841\end{array}$

$\begin{array}{lll}0.26600841 & 0.26600841 & 0.26600841\end{array}$

$\begin{array}{lll}0.26600841 & 0.26600841 & 0.26600841\end{array}$

repeated measures mixed model

R Correlation Matrix for TREE(NOMDIST) 13 


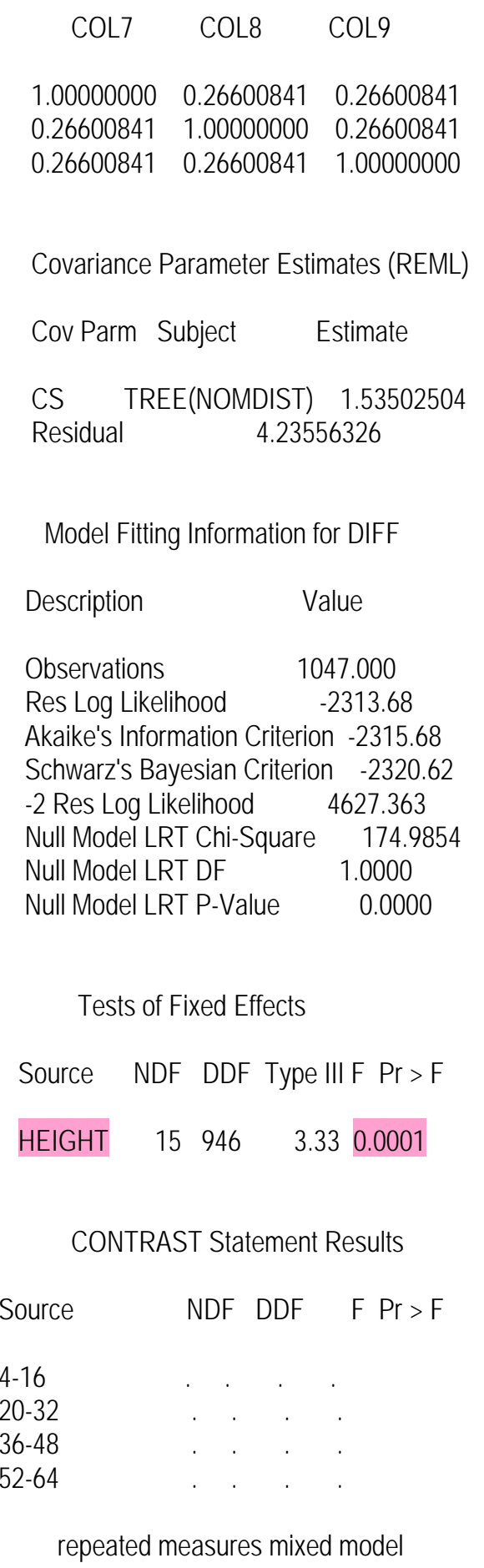




\begin{tabular}{|c|c|c|c|c|c|}
\hline Т 3.66 & 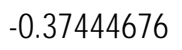 & 0.24552848 & 4 & -1.53 & \\
\hline & & 61230 & 640 & & \\
\hline & & & 66 & & \\
\hline & & & 60 & 73 & \\
\hline & & & & -1.16 & \\
\hline & & & & -2.12 & \\
\hline & & & & -1 & \\
\hline & -0.6 & 0.3 & $T$ & -0.1 & \\
\hline & -0.1 & 0.3 & 43 & -0.38 & \\
\hline & & 0.3 & & -0.39 & \\
\hline & & & & & \\
\hline & & & & & \\
\hline & & & & & \\
\hline
\end{tabular}

